
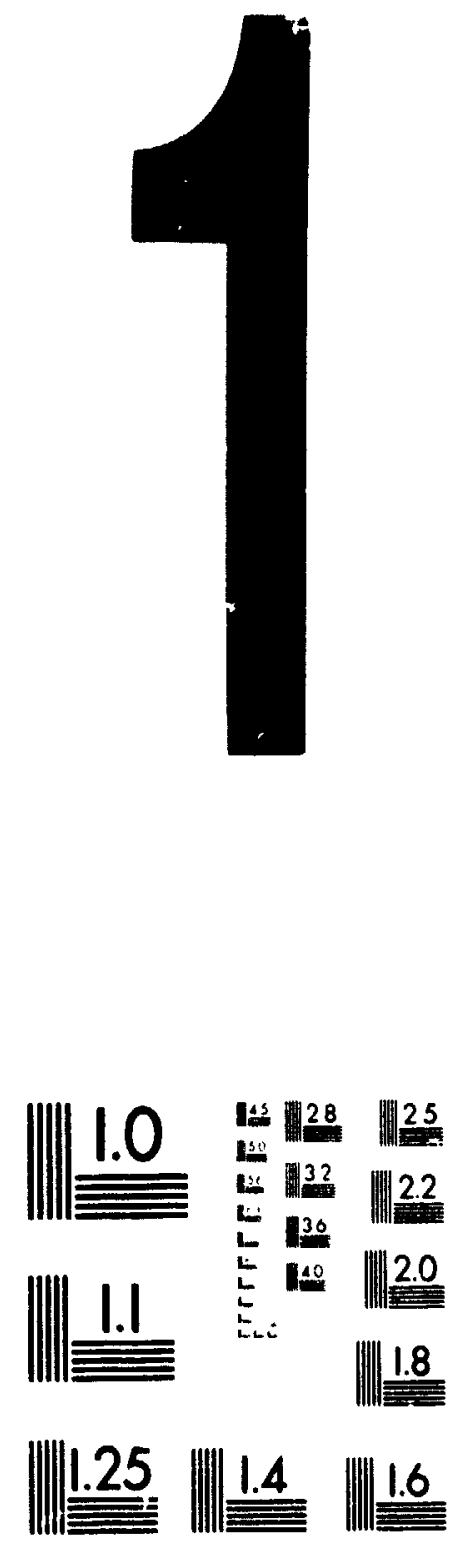

MICROCOPY RESOLUTION TEST CHART NATIONAL BUREAU OF STANDARDS STANDARD REFERENCE MATESIAL 1010a (ANSI and IS,) TEST CHART NO 21 


\section{National Library}

of Canada

Canadian Theses Service

Ottawa, Canada

KIA ON4
Bibliotheque nationale

du Canada

\section{Service des thèses canadiennes}

\section{NOTICE}

The quality of this microform is heavily dependent upon the quality of the original thesis submitted for microfilming. Every effort has been made to ensure the highest quality of reproduction possible.

It pages are missing, contact the university which granted the degree.

Some pages may have indistinct print especially if the original pages were typed with a poor typewriter ribbon or if the university sent us an inferior pholocopy.

Reproduction in full or in part of this microform is governed by the Canadian Copyright Act, R.S.C. 1970, c. C-30, and subsequent amendments.

\begin{abstract}
AVIS
La qualité de cette microlorme dépend grandement de la qualité de la thèse soumise au microtilmage. Nous avons tout fait pour assurer une qualité supérieure de reproduc tion.

S'il manque des pages, veuillez communiquer avec l'université qui a conféré le grade.

La qualité d'impression de certaines pages peut laısser à désirer, surtout si les pages originales ont été dactylographiées à l'aide d'un nuban usé ou si l'université nous a falt parvenir une photocopie de qualité inférieure.
\end{abstract}

La reproduction, méme partielle, de celte microtorme est soumise à la Loi canadienne sur le droil d'auteur. SHC 1970. c. C-30, et ses amendements subséquents 


\title{
Diversity measurements for indoor radio communications
}

\author{
by \\ Jean-François Lemieux, B. Eng, RMC
}

\author{
A thesis submitted to the \\ Faculty of Graduate Studies and Research \\ in partial fulfilment of the requirements \\ for the degree of \\ Master of Engineering
}

Ottawa-Carleton Institute for Electrical Engineering

Faculty of Engineering

Department of Systems and Computer Engineering

Carleton University

January, 1990

(C) copyright

1990, Jean-François Lemieux 
National Library

of Canada

Canadian Theses Service

Ottawa. Canada

KIA ONA

\section{Bıblıothèque nationale}

du Canada

Service des theses canadiennes
The author has granted an i.revocable nonexclusive licence allowing the National Library of Canada to reproduce, loan, distribute or sell copies of his/her thesis by any means and in any form or format, making this thesis available to interested persons.

The author retains ownership of the copyright in his/her thesis. Neither the thesis nor substantial extracts from it may be printed or otherwise reproduced without his/her permission.
L'auteur a accordé une licence irrévocable et non exclusive permettant à la Bibliothéque nationale du Canada de reproduire, preter. distribuer ou vendre des copies de sa thèse de quelque manière et sous quelque forme que ce soit pour mettre des exemplaires de cette thèse à la disposition des personnes intèressées.

L'auteur conserve la propriété du droit d'auteur qui protège sa thèse. Ni la thèse ni des extraits substantiels de celle-ci ne doivent être imprimés ou autrement reproduits sans son autorisation. 
The undersigned hereby recommends to the Faculty of Graduate Studies and Research acceptance of the thesis,

"Diversity measurements for indoor radio communications"

submitted by Jean-François Lemieux, in partial fulfilment of the requirements for the degree of Master of Engineering.

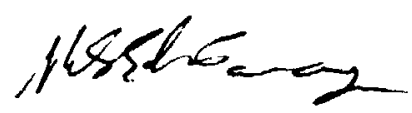

Dr. Mohamed El-Tanany Thesis Co-supervisor

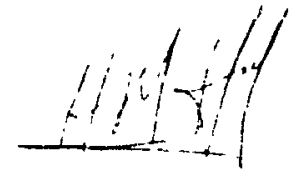

Dr. H. M. Hafez

Thesis Co-supervisor

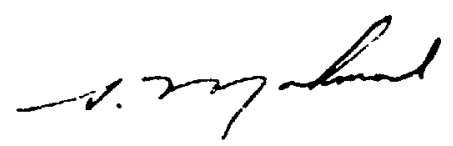

Dr. S. A. Mahmoud

Chairman,

Department of Systems and

Computer Engineering

Department of Systems and Computer Engineering

Faculty of Engineering

Carleton University

January, 1990 


\section{Abstract}

Signal propagation for the indoor radio channel has been shown to suffer from multipath fading and strong attenuation. In this work, a transmitter and diversity receiver were designed and implemented to characterize the indoor radio channel using trequency, space and polarization diversity. Selection combining was used. Statistical analysis of the data gathered was performed and envelope level distributions were calculated. Frequency diversity results showed that maximum improvement occurs with any frequency spacing above $10 \mathrm{MHz}$. Partial improvement occurs with a spacing of $5 \mathrm{MHz}$. Space diversity results showed that maximum improvement occurs with any antenna spacing above $3 / 4$ wavelength. Polarization diversity results showed that partial improvement is achieved, although smaller than that achievable with two uncorrelated channels. Attenuation results were presented in the form of the exponent of the distancepower law: it was between 3.56 and 3.49, in agreement with published results for similar types of building construction. 


\section{Acknowledgements}

I wouid like to thank my supervisor, Dr Mohamed El-Tanany, for his help, guidance and patience throughout the many months involved in conducting the experiments and writing this thesis. My wife France deserves also more credit than simple words can express for her help, understanding and moral support during the sometimes difficult periods that led to the production of this thesis. 


\section{Table of Contents}

Abstract $\ldots \ldots \ldots \ldots \ldots \ldots \ldots \ldots \ldots \ldots$ iii

Acknowledgements $\ldots \ldots \ldots \ldots \ldots \ldots \ldots \ldots$ iv

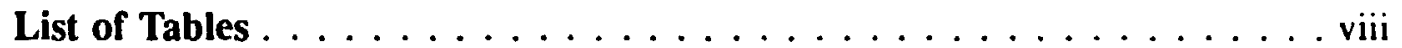

List of Figures $\ldots \ldots \ldots \ldots \ldots \ldots \ldots \ldots \ldots \ldots$ ix

List of Photographs $\ldots \ldots \ldots \ldots \ldots \ldots \ldots \ldots \ldots \ldots$

Chapter 1 Introduction $\ldots \ldots \ldots \ldots \ldots$

Section $1.1 \quad$ General $\ldots \ldots \ldots \ldots \ldots \ldots$

Section $1.2 \quad$ Fading channel . . . . . . . . . . . . . 2

Section $1.3 \quad$ Diversity $\ldots \ldots \ldots \ldots \ldots \ldots \ldots$

1.3.1 General $\ldots \ldots \ldots \ldots \ldots$

1.3.2 Combining methods $\ldots \ldots \ldots \ldots$

Section $1.4 \quad$ Thesis objectives . . . . . . . . . . . . 11

Section $1.5 \quad$ Organization $\ldots \ldots \ldots \ldots \ldots \ldots$

Chapter $2 \quad$ Review of current research $\ldots \ldots \ldots \ldots$

Section $2.1 \quad$ Introduction $\ldots \ldots \ldots \ldots \ldots$

Section $2.2 \quad$ Cunent results $\ldots \ldots \ldots \ldots \ldots \ldots$

Section $2.3 \quad$ Review of previous experiments . . . . . . . . . . 21

Section $2.4 \quad$ Experiment outline . . . . . . . . . . . . . 24

C.tapter 3 Description of experimental installations and procedures . 26

Section $3.1 \quad$ Introduction . . . . . . . . . . . . 2ú

Section $3.2 \quad$ Preliminary measurements installation . . . . . . . 27

3.2.1 Transmitter and Receiver. . . . . . . . . . 27

$3.2 .2 \quad$ Lessons learned . . . . . . . . . . . . . . 29; 
Section $3.3 \quad$ Frequency diversity transmitter . . . . . . . . . . 29

Section 3.4 Space and polarization diversity transmitter . . . . . . . . 34

Section $3.5 \quad$ Frequency diversity receiver $\ldots \ldots \ldots \ldots$

Section $3.6 \quad$ Space and polarization diversity receiver . . . . . . . . . 40

Section $3.7 \quad$ Antennas $\ldots \ldots \ldots \ldots \ldots \ldots$

Section $3.8 \quad$ Analog-to-digital converter . . . . . . . . . . 42

3.8.1 General . . . . . . . . . . . . 42

3.8.2 Modes of operation of the analog-to-digital converter . . . 43

Section $3.9 \quad$ HP Vectra PC ................ 47

3.9.1 General . . . . . . . . . . . . 47

3.9.2 Data acquisition and conversion programs . . . . . . 48

Section $3.10 \quad$ Calibration $\ldots \ldots \ldots \ldots \ldots \ldots$

Section 3.11 Measuremants procedure ............. 52

3.11.1 General ................... 52

$3.11 .2 \quad$ Procedures $\ldots \ldots \ldots \ldots \ldots$

Chapter $4 \quad$ Analysis and results $\ldots \ldots \ldots \ldots \ldots$

Section $4.1 \quad$ Introduction $\ldots \ldots \ldots \ldots \ldots \ldots$

Section $4.2 \quad$ Analysis .................. 56

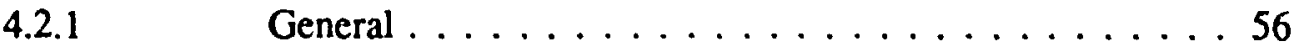

4.2.2 Processing for cumulative distribution ........ 56

4.2.3 Channel 1 correction $\ldots \ldots \ldots 60$

4.2.4 Processing for attenuation results ..........60

Section $4.3 \quad$ Diversity systems measurements $\ldots \ldots \ldots 6$

4.3.1 General $\ldots \ldots \ldots \ldots$.............. 62

4.3.2 Frequency diversity ............. 66

$4.3 .3 \quad$ Space diversity $\ldots \ldots \ldots \ldots \ldots$

4.3.4 Polarization diversity ............ 73 
Section $4.4 \quad$ Attenuation measurements $\ldots \ldots \ldots \ldots$

Section $4.5 \quad$ Summary $\ldots \ldots \ldots \ldots \ldots \ldots$

Chapter 5 Conclusion and Recommendations . . . . . . . . . . . 80

Section $5.1 \quad$ Conclusion $\ldots \ldots \ldots \ldots \ldots \ldots$. . . . . .

Section $5.2 \quad$ Contribution $\ldots \ldots \ldots \ldots \ldots \ldots \ldots$

Section $5.3 \quad$ Recommendations for future research $\ldots \ldots \ldots \ldots 1$

Bibliography ...........................

Appendix A Program Listings . . . . . . . . . . . . . . . 94

Section $A .1 \quad$ ADSAMP.BAS $\ldots \ldots \ldots \ldots \ldots$

Section A.2 DATASCALE .................... 99

Section A.3 DATAVG $\ldots \ldots \ldots \ldots \ldots \ldots \ldots \ldots$

Section A.4 DATACDF ................ 108

Section A.5 $\quad$ LOSS $\ldots \ldots \ldots \ldots \ldots \ldots \ldots$

Appendix B File Format . . . . . . . . . . . . . . 117

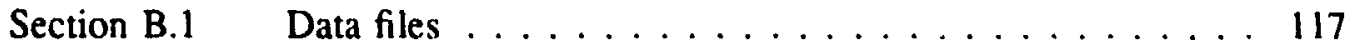

Section B.2 Result Sles . . . . . . . . . . . . . . . 117

Section B.3 Result matrix files . . . . . . . . . . . . 118

Appendix C DASH-16F .................... 119

Section C.. Features and options . . . . . . . . . . 119

Section C.2 Data acquisition modes . . . . . . . . . . . . . 120

Appendix D Scatter Diagrams . . . . . . . . . . . . . . 123

Appendix E Measurement area and Conditions . . . . . . . . . . $12 \mathrm{R}$

Section E.1 Measurement locations .............. 128

Section E.2 Measurement conditions . . . . . . . . . . . 130

Section E.3 Hardware stability . . . . . . . . . . . . . 130 


\section{List of Tables}

Table $3.1 \quad$ Transmitter maximum output power $\ldots \ldots \ldots$. . . . . . 31

Table 3.2 List of DASH-16F modes used in the exper.ment . . . . . . . 44

Table 4.1 Frequency diversity improvement values . . . . . . . . 66

Table $4.2 \quad$ Space diversity improvement values $\ldots \ldots \ldots \ldots$

Table $4.3 \quad$ Polarization diversity improvement . . . . . . . . . . . 74

Table 4.4 Exponents of distance-power law for all diversity cases . . . . 75

Table E.1 Locations and distances . . . . . . . . . . . . . . 128 


\section{List of Figures}

Figure $1.1 \quad$ Vectorial sum of received signals $\ldots \ldots \ldots \ldots$

Figure $1.2 \quad$ Multipath intensity profile $\phi_{c}(\tau) \ldots \ldots \ldots$

Figure 3.1 Block diagram of preliminary transmitter . . . . . . . . 29

Figure $3.2 \quad$ Block diagram of prelimirary receiver $\ldots \ldots \ldots . \ldots 28$

Figure 3.3 Block diagram Frequency diversity transmitter . . . . . . . 30

Figure 3.4 Power spectrum of frequency diversity transmitter output, : $\mathrm{MH} \mathrm{I}_{2}$

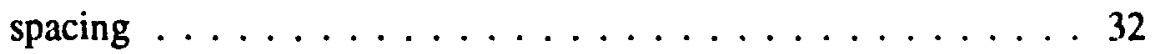

Figure 3.5 Power spectrum of frequercy diversity transmitter output, $5 \mathrm{MHz}$ spacing ........................... 32

Figure 3.6 Power spectrum of frequency diversity transmitter output, $10 \mathrm{MHz}$ spacing ............................. 33

Figure 3.7 Power spectrum of frequency diversity transmitter output, $15 \mathrm{MHz}$

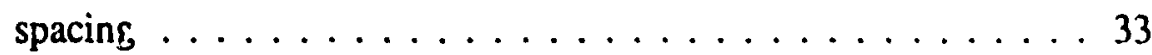

Figure 3.8 Power spectrum of frequency diversity transmitter output, $20 \mathrm{MHz}$ spacing ......................... 34

Figure 3.9 Block diagram of the space/polarization diversity transmitter . . 35

Figure $3.10 \quad$ Block diagram of frequency diversity receiver . . . . . . . . 36

Figure 3.11 Transfer function of receive crystal filter channel $1 \ldots \ldots 38$

Figure 3.12 Transfer function of receive crystal filter channel $2 \ldots \ldots$. . . . 38

Figure 3.13 Output of crystal filter, frequency diversity receiver,channel 1 . 39

Figure 3.14 Output of crystal filter, frequency diversity receiver, channel $2 \ldots 39$

Figure 3.15 Block diagram of space and polarization diversity receiver . . . 40

Figure 3.16 Calibration curve, $1 \mathrm{MHz}$ spacing frequency diversity . . . . . . 51

Figure 3.17 Calibration curve, space and polarization diversity . . . . . . 51

Figure 4.1 Envelope detector output, frequency diversity, $1 \mathrm{MHz} \ldots \ldots .3$

Figure 4.2 Envelope detector output, frequency diversity, $20 \mathrm{MHz}$ spacing . 64

Figure 4.3 Envelope detector output, space diversity, $75 \lambda$ spacing . . . . 64

Figure 4.4 Envelope detector output, space diversity, $2 \lambda$ spacing . . . . . 65 
Figure 4.5 Envelope detector outnut, polarization diversity . . . . . . . 65

Figure 4.6 Cumulative distribution function for $1 \mathrm{MHz}$ spacing . . . . . . 67

Figure 4.7 Cumulative distribution function for $5 \mathrm{MHz}$ spacing $\ldots \ldots$. . . 68

Figure $4.8 \quad$ Cumulative distribution function for $10 \mathrm{MHz}$ spacing $\ldots \ldots$. . 68

Figure 4.9 Cumulative distribution function for $15 \mathrm{M}: \mathrm{Hz}$ spacing . . . . . 69

Figure 4.10 Cumulative distribution function for $20 \mathrm{MHz}$ spacing . . . . . 69

Figure 4.11 Curauls:ive distribution function for $.75 \lambda$ spacing $\ldots \ldots \ldots 71$

Figure 4.12 Cumulative distribution function for $1 \lambda$ spacing . . . . . . 71

Figure 4.13 Cumulative distribution function for $1.5 \lambda$ spacing . . . . . . 72

Figure 4.14 Cumula :ve distribution function for $1.66 \lambda$ spacing $\ldots \ldots \ldots 72$

Figure 4.15 Cumulative distribution function for $2 \lambda$ spacing $\ldots \ldots \ldots \ldots$

Figure 4.16 Cumulative distribution function for $90^{\circ}$ spacing . . . . . . . . 74

Figure 4.17 Scatter diagram for frequency diversity $1 \mathrm{MHz}$, all locations . . 77

Figure $4.18 \quad$ Scatter diagram for space diversity .75 $\lambda$, all locations . . . . . . 77

Figure 4.19 Scatter diagram for polarization diversity, all locations . . . . . . 78

Figure D.1 Scatter diagram for frequency diversity $5 \mathrm{MHz}$, all locations . . 123

Figure D.2 Scatter diagram for frequency diversity $10 \mathrm{MHz}$, all locations . 124

Figure D.3 Scatter diagram for frequency diversity $15 \mathrm{MHz}$, all locations . 124

Figure D.4 Scatter diagram for frequency diversity $20 \mathrm{MHz}$, all locations . 125

Figure D.5 Scatter diagram for space diversity $1 \lambda$, all locations . . . . 125

Figure D.6 Scatter diagram for space diversity $1.5 \lambda$, all locations . . . . 126

Figure D.7 Scatter uiagram for space diversity $1.66 \lambda$, all locations . . . 126

Figure D.8 Scatter diagram for space diversity $2 \lambda$, all locations . . . . 127

Figure E.1 Floor plan of the measurement area and locations position . . 129 


\section{List of Photographs}

Plate $1 \quad$ Frequency diversity transmitter $\ldots \ldots \ldots \ldots$

Plate $2 \quad$ Frequency diversity receiver . . . . . . . . . . . . 84

Plate $3 \quad$ Space and polarization diversity receiver . . . . . . . 85

Plate $4 \quad$ Example of antenna used . . . . . . . . . . . . 86

Plate $5 \quad$ Holding frame for space diversity antenna . . . . . . . 87

Plate $6 \quad$ Holding frame for polarization diversity antenna . . . . . 88

Plate $7 \quad$ Guiding frame used to take measurement . . . . . . . . . 89 


\section{Chapter 1 Introduction}

\subsection{General}

Mobile communication is increasingly becoming a part of our society. Paging is a well known and accepted method of reaching somebody on the move. Over the past few years, cellular telephone systems have experienced phenomenal growth and are becoming an indispensable tool for more and more people. Beginning as a vehicle based system, the latest developments in the cellular telephone industry are bringing on the market portable cellular telephones. Research and development specifically on indoor radio propagation and communication systems came from the development of these portable telephones.

Indoor radio communication, sometimes called indoor wireless communication, does not have any major practical applications yet but that time is fast approaching. The cordless telephones pr. ntly available are more a gadget than a new communication system. Their restric quencies and crude features will prevent them from having any serious future $[1],[2]$. They are only an early application of a technology still in its infancy. The possible implementations of indoor wireless include narrowband systems for voice or low speed data as with a wireless PEX [3] or for communication and control in factories [4], [5], [6]. They also include wideband systems for high rate data transmission, possibly in a wireiess LAN [7]. 
It is tempting to use the cellular telephone system and its popuiarity as a possible example for the future of indoor radio communication. Just as cellular radio fulfilled a need for quality, high capacity mobile telephone communication, indoor radio could mature along similar lines to provide portable and flexible comınunication within a large building or factory. Integrated systems where one portable phone will allow its user to make calls from any office building or public place are not that far fetched [1], [2].

Market penetration of the indoor radio technology will be linked more or less directly to its cost. As any other high technology application, cost reduction will come with mass production of simple and robust instruments. The key to this simplicity is a better knowledge of the channel and its characteristics. All types of radio channel have some common elements but the frequencies and the in-building environment of the indoor radio channel bring a number of unknowns that must be explored.

\subsection{Fading channel}

The indoor radio channel is not a friendly environment for signal propagation. All kinds of obstacles (people, walls, furniture etc) attenuate and reflect a signal to various degrees. At any given location, the received signal is the sum of many replicas of the transmitted signal that propagated along different paths. These obstacles, the paths and the propagation conditions in general are moving and changing with time. The changes in the channel transfer function have a great influence on communication systems performance. They alter the amplitude and the phase of the received signal. It is convenient although somewhat imprecise to separate these changes in two groups based on their time scale: short-term and long-term fading. Communication links experience variations in performance most'y due to the effects of short term fading. These effects are usually described in terms of signal-to-noise ratio and probability of error. Longer term variations of the channel structure are also taking place. Looking at it from the point of view of statistical communication theory, the short term fading is concerned with the instantaneous values of parameters described by longer term statistics [8]. We will review short term fading and will show which statistical model can be used to explain it. 


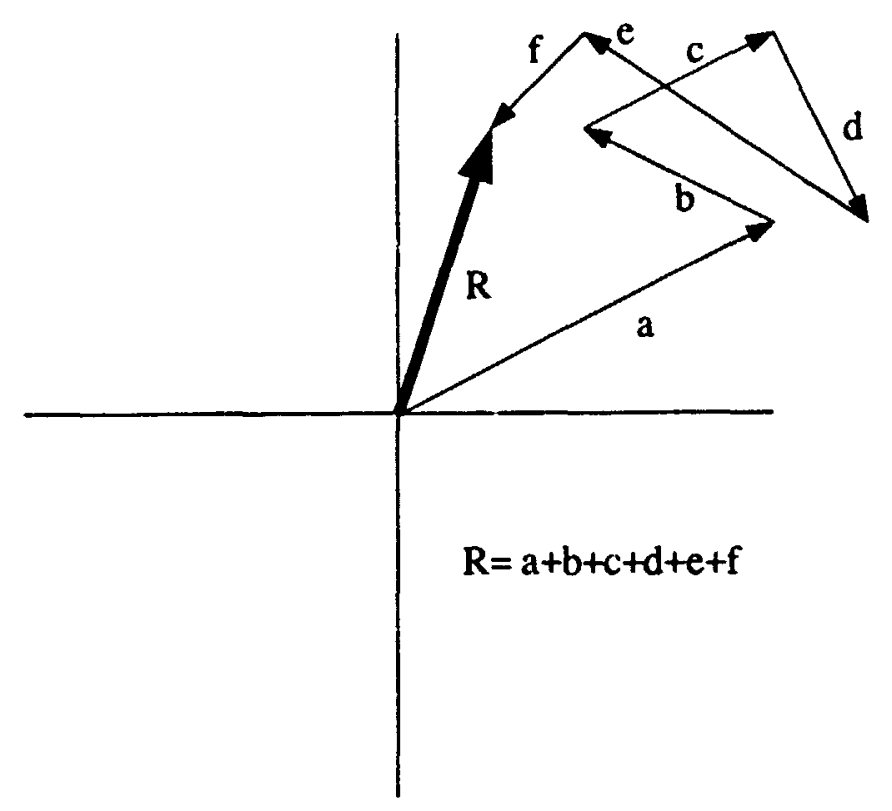

Figure- 1.1: Vectorial sum of received signals

Short term fading can also he called multipath fading because the energy is arriving at the receiver along several distinct paths. If either the receiver position or the elements aifecting those paths undergo changes, it will produce a moving pattern of signal maxima and minima that causes short term fading. The arrival of the signal along these multiple paths is caused by changes in the physical medium that cause scattering and ray splitting and by reflections on various objects. Each path provides a component of the resulting signal with its own amplitude and phase. Even if all components are from the same source, the small variations in path length caused by reflections, scattering and medium changes may amount to lirge changes in the electrical phase of the received signal. Figure 11 shows this concept in vector format. It is easy to see that the resulting vector can have sudden changes in amplitude with small variations in the phise of some components. This is called destructive interference: opposing phase vectors of roughly the same intensity added together can result in a signal of very small magnitude. The phase and attenuation of arriving rays is changed randomly over the different paths. 
The effect of multipath fading can be studied mathematically. Ising the complex low pass equivalent notation as in [9], the transmitted signal is

$$
s(t)=\operatorname{Re}\left[\begin{array}{ll}
u(t) & c^{j \omega_{c} t}
\end{array}\right]
$$

where $u(t)$ is the complex low pass envelope and $\omega_{c}$ is the angular carrier frequency. Each of the multiple propagation paths has a certain attenuation and a certain phase. The received signal is :

$$
x(t)=\sum_{n} a_{n}(t) s\left(t-\tau_{n}(t)\right)
$$

for a discrete numbers of paths and

$$
x(t)=\int_{-\infty}^{\infty} a(t ; \tau) s(t-\tau) d \tau
$$

for a continuous number of paths (e.g. HF propagation); where $a_{n}(t)$ is the attenuation and $\tau_{n}(t)$ is the delay associated with the $n$th path. $a(t ; \tau)$ is the attenuation of signal components at delay $\tau$ and time t. To sinplify the expression, assume there is a finite number of paths with a fixed attenuation and delay. This is an idealized static situation where neither the receiver nor any scatterer/reflector are moving and the propagation medium is changing slowly enough to be considered static. With this assumption, 1.2 becomes

$$
x(t)=\sum_{n} a_{n} s\left(t-T_{n}\right)
$$

or substituting 1.1 in 1.4

$$
x(t)=\operatorname{Re}\left[\sum_{n} a_{n} u\left(t-\tau_{n}\right) c^{j \omega_{c}\left(t-\tau_{n}\right)}\right]
$$

This equation can be rearranged to show the equivalent low pass received signal envelope:

$$
x(t)=\operatorname{Rc}\left[r(t) \quad c^{j \omega_{c} i}\right]
$$

where $r(t)$ is:

$$
r(t)=\sum_{n} a_{n} u\left(t-\tau_{n}\right) e^{-j \omega_{c} \tau_{n}}
$$


or for the continuous case:

$$
r(t)=\int_{-\infty}^{\infty} a(\tau) u(t-\tau) e^{-j \omega_{c} \tau} d \tau
$$

In the case of an unmodulated carrier at $f=t_{c}, u(t)=1$ for all $t$ and $r(t)$, the signal envelope, is indeperdent of time.

$$
r(t)=\sum_{n} a_{n} e^{-j \omega_{c} \tau_{n}}
$$

This is the case shown in Figure 1.1 where each path cor.tributes to the resultant signal. If the scatterers are moving and/or if the receiver is moving, the attenuation and delay will be constantly changing. It is reasonable to assume in this case that the attenuation and delay would be changing in a random fashion. Each path component is a random variable. When there are large numbers of paths, the central limit theorem can be applied and $r(t)$ can be modeled as a zero mean complex gaussian random process. The received signal envelope can be expressed as:

$$
r(t)=R+j S
$$

where:

$$
\begin{gathered}
R=\sum_{n} a_{n} \cos \omega_{c} \tau_{n} \quad \text { and } \\
S=\sum_{n} a_{n} \sin \omega_{c} \tau_{n}
\end{gathered}
$$

$r(t)$ can be also expressed in the polar form as:

$$
r(t)=A(t) e^{(-j \psi(t))}
$$

with

$$
\begin{gathered}
A(t)=\sqrt{R^{2}+S^{2}} \text { the envelope and } \\
\psi(t)=\tan ^{-1} \frac{S}{R} \text { the phase }
\end{gathered}
$$

For a zero mean complex Gaussian process, the joini density function in $R$ and $S$ is a bivariate Gaussian distribution:

$$
\begin{gathered}
p(R, S)=\frac{1}{2 \pi \sigma^{2}} \operatorname{cxp}\left(-\frac{R^{2}+S^{2}}{2 \sigma^{2}}\right) \\
\sigma^{2}=\frac{1}{2} E\left\{\left|r^{2}\right| \text {. mean power in waveform }\right\}
\end{gathered}
$$


The joint probability density function (pdf) of the random variables $A(t)$ and $\psi(t)$ (from 1.13) can be obtained by a transformation of 1.14. By integrating the transformed joint pdf aver each variable, a pdf for the envelope and a pdf for the phase is obtained:

$$
\begin{gathered}
p(\psi)=\frac{1}{2 \pi} \quad 0 \leq \psi \leq 2 \pi \\
p(A)=\frac{A}{\sigma^{2}} \operatorname{cxp}\left(\frac{-A^{2}}{2 \sigma^{2}}\right) \quad 0 \leq A \leq \infty
\end{gathered}
$$

The distribution obtained from the envelope density function is a Rayleigh distribution with 0 mean. The term Rayleigh fading is often attached to those channels or signals displaying this type of envelope behavior. The distribution obtained from the phase density function is a uniform distribution. These two distributions are widely known and discussed in many manuals and published documents. This is the most usual model of envelope and phase distribution in mobile radio. It is a valid model when all path attenuations and delays are zero mean random variables, for small values of envelope and when there are enough scatterers to spread the phase unifonnly. When there is a single non-fading component in addition to a number of fading ones, the envelope has a Rice distribution. Its pdf is:

$$
\begin{aligned}
& p(A)=\frac{A}{\sigma_{f}^{2}} \exp \left(-\frac{2 \sigma_{0}^{2}+A^{2}}{2 \sigma_{f}^{2}}\right) I_{0}\left(\frac{A \sqrt{2} \sigma_{0}}{\sigma_{f}^{2}}\right) \quad 0 \leq A \leq \infty \\
& \sigma_{f}^{2}=\text { mean power of fading component } \\
& \sigma_{0}^{2}=\text { mean power of non - fading component }
\end{aligned}
$$

The topic of frequercy selective fading will now be addressed. Equation 1.8 can be seen as the con olution of $u(t)$ with the equivalent low pass channel impulse response $c(\tau, t):$

$$
c(\tau, \ddot{i})=a(\tau, t) e^{-j \omega_{c} \tau}
$$

From this impulse response, the channel transfer function can be obtained by doing a Fourrier transform on $\mathrm{c}(\tau, \mathrm{t})$ in the $\tau$ variable:

$$
C(f, t)=\int_{-\infty}^{\infty} c(\tau, t) c^{-\jmath 2 \pi f \tau} d \tau
$$


The autocorrelation function obtained from $\mathrm{C}(\mathrm{f}, \mathrm{t})$ is called $\phi_{\mathrm{C}}$ :

$$
\phi_{C}\left(f_{1}, f_{2} ; \Delta t\right)=\frac{1}{2} E\left\{C^{*}\left(f_{1} ; t\right) C\left(f_{2} ; t+\Delta t\right)\right\}
$$

Under the assumption of wide sense stationarity of the channel and that scattering at $\tau_{1}$ and $\tau_{2}$ is uncorrelated, $\phi_{C}$ is a function only of $\Delta f=f_{1}-f_{2}$ and not of a specific frequency. $\phi_{C}(\Delta f ; \Delta t)$ is the spaced-frequency spaced-time correlation function. From this function, many other functions can be derived.

For $\Delta t=0, \phi_{C}(\Delta f ; 0) \equiv \phi_{C}(\Delta f)$. This autocorrelation in the frequency variable gives a measure of the coherence bandwidth of the channel, $\Delta f_{c}$. Practically speaking, it means that two tones spaced by more than $\Delta f_{c}$ will be affected differently by the channel. This is the basis of frequency diversity. If $\Delta f_{c}$ is smaller than the transmitted signal bandwidth, the received signal will be very distorted: this is frequency selective fading. If it is the other way around, this is frequency non-selective fading or often called flat fading. For $\Delta \mathrm{f}=0, \phi_{C}(0 ; \Delta \mathrm{t}) \equiv \phi_{\mathrm{C}}(\Delta \mathrm{t})$. This function of $\Delta \mathrm{t}$ gives a measure of the coherence time $\Delta t_{c}$ of the channel. The concept is similar to the coherence bandwidth just discussed. This coherence time is an indication that two signals at the same frequency will be affected differently by the channel if transmitted at a time interval $>\Delta t_{c}$. This is the basis of time diversity.

Since $C(f, t)$ is the Fourrier transform of $c(\tau, t)$, the inverse Fourrier transform of $\phi_{C}(f ; \Delta t)$ is $\phi_{C}(r ; \Delta t)$ which is by definition the autocorrelation of $c(\tau, t)$ :

$$
\phi_{c}(\tau ; \Delta t)=\frac{1}{2} E\left\{c^{*}\left(\tau_{1} ; t\right) c\left(\tau_{2} ; t+\Delta t\right)\right\}
$$

The inverse Fourrier transform of $\phi_{C}(f ; 0)$ is then $\phi_{c}(\tau ; 0)$. This is the average power output of the channel as a function of the delay $\tau$. It is also called the multipath intensity profile of the channel. The multipath spread of the channel $T_{m}$ is the range of $\tau$ for which $\phi_{c}(\tau)$ is non zero. Figure 1.2 shows a typical profile. This means that for delay $\tau>T_{m}$, the delayed versions of the transmitted signal are too weak to be of any significance. By way of its inverse Fourrier transform relation to $\phi_{C}(f), \frac{1}{T_{m}} \approx \Delta f_{c}$, the coherence bandwidih. $T_{m}$ is easier to measure than the coherence bandwidth. Saleh 
and Valenzuela [10] and Devasirvatham [11] explain the jpocedure and give results of such measurements.

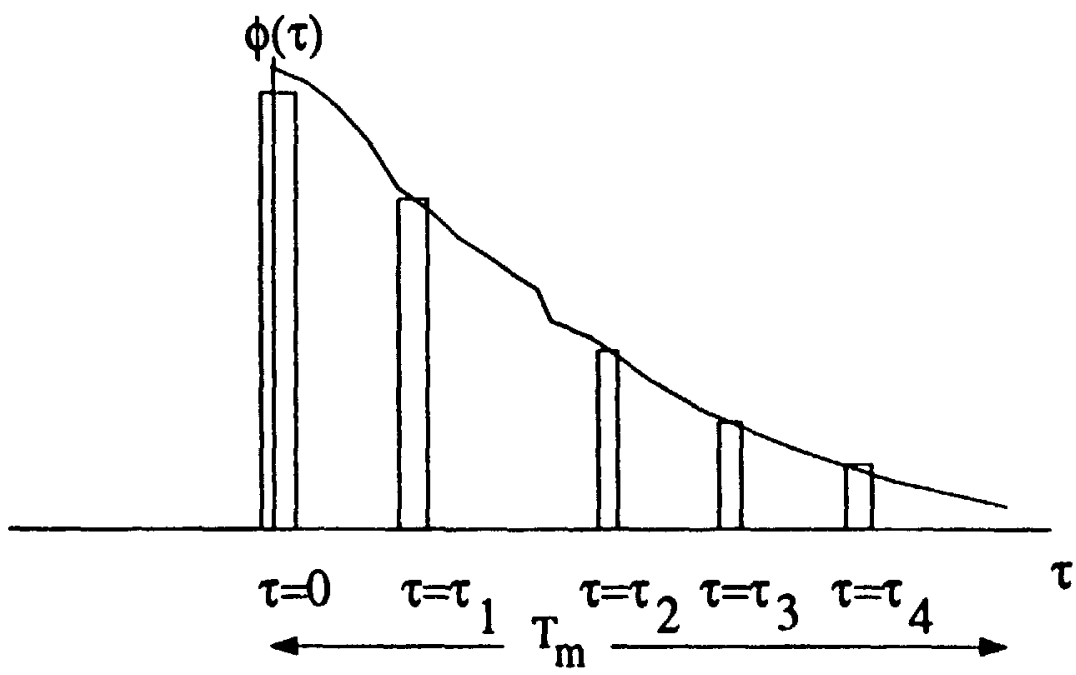

Figure- 1.2: multipath intensity profile $\phi_{c}(\tau)$

\subsection{Diversity}

\subsubsection{General}

Diversity combining has been in use for many years in the world of $\mathrm{HF}$ radio, with the first experiments reported in the late twenties [12], [8]. It is based on the fact that if two or more independently fading transmission paths carry the same message, the probability that all paths will experience a deep fade at the same time is lower than for a single path. There are a few methods widely used either to choose the path with the strongest signal or to combine the signals from each path to make a stronger one. The original method to realize independent fading paths consisted in using more than one receiving antenna separated by some distance. The distance between each pair of antennas had to be large enough to insure independent fading. Other methods used to achieve independent fading consisted of transmitting on different frequencies, at different time or with different polarizations. There is no limit on the number of diversity branches 
that can be used but the amount of improvement decreases with every additional branch. Performance improvement must then be balanced with receiver complexity.

Space diversity is a simple technique which does not require additional frequency spectrum. A spacing between each pair of antennas of at least $\lambda / 2$ [12] should be sufficient. Practically, the required spacing depends of the position of the scatterers causing the multipaths.

Frequency diversity requires more spectrum but uses only one antenna at the receiver. The frequency separation will depend on the coherence bandwidth of the channel.

Polarization diversity takes advantage of the de-polarization of the transmitted signal as it propagates through the channel. A vertically polarized signal leaving the transmitter will have both $v$ :rtical and horizontal components when it reaches the receiver. The physical process of de-polarization or cross-polarization is very complex and is not within the scope of this work. Polarization diversity brings in focus problems associated with radiation patterns and type of antenna. A dipole antenna has a null along any plane containing the antenna. A vertical dipole has uniform azimuthal directivity. By turning it $90^{\circ}$, there are two directions from which no significant energy will be collected [13].

\subsubsection{Combining methods}

Some techniques must be used to tum the independent fading in the branches of the receiver into an improvement in performance. Combining is the mechanism used to do this. There are three principal combining methods: selection, maximal ratio and equal gain combining. When using linear detection for demodulation, there is no difference between doing the combining before or after detection. When using nonlinear detection (e.g. square law), predetection combining yields a $3 \mathrm{~dB}$ gain over post-detection combining [14].

Selection combining consists of selecting the branch with the best signal among all branches. In predetection combining, this branch is used for demodulation. In post-detection combining, the signal from this branch is sent to the receiver output. The algorithm consists of monitoring all branches and picking the strongest. When 
the signal of the selected branch falls lower than any other one, a new best signal is selected. At first this method seems very simple but is difficult to implement because the threshold where a decision to switch branch is made is always changing. Reference [12] shows a $10 \mathrm{~dB}$ improvement between 1 branch and 2 branches for $P_{12}=1 \%\left(P_{12}\right.$ is the probability that either signal in branch 1 or 2 is lower than a given level $\mathrm{L}$ ). For $P_{12}=.1 \%$ the improvement is almost $15 \mathrm{~dB}$. A $10 \mathrm{~dB}$ improvement means that for the same probability, $L$ is $10 \mathrm{~dB}$ higher with 2 branch diversity than with no diversity.

Switched combining is an easier method to implement. The signal from one branch is selected until it becomes lower than a fixed threshold $A$. When this happens, the next branch is selected. Hopefully its signal is higher than $A$. The performance improvement from this method is at best equal to selection combining. This happens only close to the threshold level. At all other points, it is worse than selection combining but it is still better than no diversity.

Maximal ratio combining consists of weighting the signal from each branch according to its level and then summing them all. Before summing them, all signals are co-phased. Ideally, the strongest signal, having the greatest weight, contributes the most to the final signal and the weakest signal contributes the least. For $P_{12}=1 \%$ the improvement from 1 to 2 branch is $11.5 \mathrm{~dB}$ as shown in reference [12]. For $P_{12}=.1 \%$ the improvement is $17 \mathrm{~dB}$. This method requires complex circuitry at the receiver to obtain the correct weighting factors and to phase align all signals.

Equal gain combining is a variation of maximal ratio combining. The signals are co-phased before summing but each one is given the same weight. The performance improvement is generally only a fraction of a dB lower than maximal ratio combining. Taking the limit for an infinite number of branches, the difference is $1.05 \mathrm{~dB}$ [12].

The results mentioned earlier were for a 2 branch receiver where the branches were uncorrelated. If they are partially correlated, the performance improvement will not be as high. Ultimately, when correlation is total, there is no improvement. In selection combining, for a 2 branch receiver at $P_{12}=1 \%$, the improvement is $3 \mathrm{~dB}$ poorer for an envelope correlation of .8 compared to an envelope correlation of 0 . For correlation of 
.6 and .3 , the improvement is $2 \mathrm{~dB}$ and $1 \mathrm{~dB}$ poorer respectively. With maximal ratio combining, the improvement is about $1 \mathrm{~dB}$ poorer for a correlation of $.3,2 \mathrm{~dB}$ for a correlation of .6 and about $4 \mathrm{~dB}$ poorer for a correlation of .8 .

\subsection{Thesis objectives}

The objectives of this thesis are threefold:

1. Design and implementation of an experimental installation for the characterization of the indoor portable channel using C.W. transmission. The transmitter section should be capable of transmitting one or two tones with variable frequency spacings. Its output power should also be variable. The receiver section is to be designed such that it allows for the individual reception of two channels simultaneously. The receiver antenna system is to be either one monopole, two monopoles or two cross-polarized monopoles. The receiver is to be interfaced to a high speed data acquisition system to allow for the gathering and storage of data from the received channels with minimal intervention.

2. Performance of a comprehensive set of experiments, using the installations outlined above, to gather sufficient data to characterize the portable radio channel using diversity reception. The experiments are to be conducted at a set of locations that is representative of the different conditions in such places as an engineering building.

3. Analysis on a statistical basis of the large volume of data gathered from such experiments. The analysis will extract important parameters such as signal loss. signal distribution, outage probability and how this is influenced by different kind of diversity and by the level of activity in the locations where the experiments took place.

\subsection{Organization}

The remainder of this thesis is organized in four chapters. 
Chapter 2 will review previous experiments done in the area of indoor radio communication and the use of diversity. The experimental installations, procedures and results obtained in these experiments will be presented. From this review, the initial design parameters of our experiment will be defined. An outline of the experiment will be presented

Chapter 3 will describe the experimental installat ons built in the course of this thesis, the procedures used to calibrate the receivers and to record the data. Transmitters, receivers and antennas design will be described. The data acquisition system will be covered in detail.

Chapter 4 will describe the statistical analysis carried out on the recorded data. The data processing steps will be explained and the algorithms used will be described. The main type of data analysis done was the computation of average cumulative distribution functions for the signal of each branch and then for a signal obtained by doing selection combining on the recorded data. Limited results (distance-power ldw) on attenuation versus distance will be presented.

Chapter 5 will conclude by reviewing the material presented, specially the data and the results. The principal findings of this thesis will then be compared to theoretical models and to previously published results. Recommendations for further related research work will be given at the end of the chapter. 


\section{Chapter 2 Review of current research}

\subsection{Introduction}

Measurements of indoor radio propagation characteristics can be divided into narrowband measurements and wideband measurements. Narrowband experiments are usually done using continuous wave (C.W.) transmitter to get information on attenuation, coverage and signal envelope distribution. These cumulative distributions, usually done for small-scale areas, are an important tool to predict the error performance of narrowband transmission systems. The attenuation measurements must be done over a larger area to find how the signal is attenuated when propagating within a building. It is often referred to in the literature as the distance-power law. The free space received signal power has an inverse relationship to the square of the distance. Indoor propagation usually has a different exponent: most of the time it is greater e.g. 3, 4, 5 [15], [16] and sometime smaller e.g. 1.5, 1.8 [10], [17].

Wideband measurements are usually done to find out the time delay spread of the building tested. This is a measure of how many replicas of the transmitted signal will be received along the multiple paths of the building. A very short pulse (large bandwidth) is sent at a certain pulse repetition frequency. The receiver output is the summation of all received signal at that time point. A plot of this output vs time might show many distinct pulses or a large smear for every pulse transmitted. This will depend on whether there are some well defined paths or more diffused paths. The time delay 
spread is the delay between the arrival of the first and the last pulse. The shortest delay does r.Jt always mean the largest amplitude. A few strong signals can add destructively to produce a small output. The delay depends on the path traveled. In simple situations, the received signal can be traced back to a given physical reflector by using the time delay to calculate the distance traveled by the signal. The information extracted from these type of measurements is usually the power delay profile and the root mean square (rms) value of the time delay spread. These parameters have a direct impact on the maximum data rate that could be achieved for a given probability of error. Cumulative distributions of the rms time delay spread are produced. The measurement resolution will dejend on the length of the pulse used as a probe: the shorter the pulse the better the resolution. Data on signal attenuation must be recorded in the course of these experiment and is often presented.

Some authors do not directly report experimental measurements but present results from various applications of indoor communication systems [18], [19], [20], [21]. They base their work on experimental data previously reported and usually support it with computer simulations. These systems usually include many of the following aspects: modulation/demodulation, media access scheme, equalization, channel assignment, use of diversity, system availability to the user, error correction code, elimination or diminution of co-channel interference and use of antennas or slotted coaxial cable.

The next section will review currently published results of indoor radio experiments. In section 2.3, we will review the experiments and use the information to design our experiment. The experiment's outline will be described in section 2.4 .

\subsection{Current results}

In the early eighties, Hoffman and Cox [22], Cox, Murray and Norris [23] did some work on radio wave propagation and signal level distribution, both with $\mathrm{CW}$ signals. The transmitter for the first experiment was outside, illuminating a small metallic building with the receiver scanning some locations inside. In the second experiment, Cox et al were in a suburban area and had the transmitter moved around inside one house and three 
small buildings with the receiver located outside some distance away. They provided part of the data to support the concept later put forward by Cox [2] of universal portable radio communication. Both experiments reported that signal envelope variations in a small area closely followed a Rayleigh distribution. Large scale variations of the signal level followed a log-norma! distribution onse distance attenuation effects were removed.

Alexander's experiments [16], [24] are truly about indoor radio propagation with both transmitter and receiver inside the same building. A CW signal was transmitted from a portable source moved around a number of rooms on the same floor while the receiver was fixed. Signal envelope distributions were also close to a Rayleigh distribution. He also characterized distance-power laws for many types of building materials [15]. Steel partitions gave a distance-power law of 5.7, brick walls gave about 3.9 and plasterboard and open plan gave 2.5 to 2.8 .

In continuation of these experiments, Alexander and Motley [25] used a 2-branch space diversity receiver with selection sombining to see the possible improvements in two systems under consideration. The first system used two frequencies spaced by $45 \mathrm{MHz}$ to transmit and receive. The second one used burst-mode operation on one frequency where each time period was divided in a transmit and a receive period. The antenna spacing varied from $\lambda / 4$ to $\lambda$ in $\lambda / 4$ increments. At the $1 \%$ probability point (as explained in section 1.3.2: the probability that the signal in either branch is lower than a given level) an advantage of $10 \mathrm{~dB}$ was obtained, well in agreement with the theoretical improvement of selection combining. The variation in antenna spacing made no significant difference in the signal strength distribution.

Patsiokas, Johnson and Dailing [26] did in-building attenuation measurements at 150,450 and $850 \mathrm{MHz}$ with $\mathrm{CW}$ signal. They gave an histogram of signal envelope level for each frequency but no cumulative distribution. They reported attenuation versus distance for the three frequencies in the form of slopes ( $\mathrm{dB} / \mathrm{foot})$ of their attenuation versus distance graph. At $850 \mathrm{MHz}$, the slope was $.42 \mathrm{~dB} / \mathrm{foot}$ up to 70 feet then .27 $\mathrm{dB} / \mathrm{f}$ wot. They attribute the change of slope to the slower attenuation of the indirect propagation waves composing the received signal for distances over 70 feet. They 
recorded $40 \mathrm{~dB}$ fades at $850 \mathrm{MHz}$ and noted that fades were much sharper at 850 than at 450 and $150 \mathrm{MHz}$.

Devasirvatham conducted time delay spread measurements using a wideband (80 $\mathrm{MHz}$ ) signal. He reported measurements in a small and in a large building [27]. For all locations in the large building the median rms time delay spread was $120 \mathrm{~ns}$ and the worst case was 250 ns. For all locations in the small building the median ms time delay spread was $60 \mathrm{~ns}$ and the worst case was $218 \mathrm{~ns}$, a surprising result considering the large difference in building size and geometry. A large hill behind the small building might have increased its effective size as far as those measurements were concerned. As might have been expected, the effect of a good direct path reduced ms time delay spread to a smaller value. The maximum delay was $88 \mathrm{~ns}$ in the small building and 70 ns in the large one. This effect was also noted in [11] where he made measurements on an inside to outside path from two houses in a suburban area (same location as in [23]). When there was no direct path from the van (receiver) to the house (transmitter), the maximum time delay spread was $422 \mathrm{~ns}$ and the minimum was $2.20 \mathrm{~ns}$. With a good direct path, the maximum delay was under 100 ns. Attenuation measurements were also made by calculating the total power of each profile. With an $80 \mathrm{MHz}$ wide signal, the measurement gave an average over a large bandwidth. In reference [11] they were in agreement with measurements reported by Cox et al [23] considering the large uncertainties of such measurements. It was noted that for longer time delays the signal strength was lower in the small building than in the large one.

Saleh and Valenzuela [10] conducted wideband measurements in a medium size two-story office building. They used $10 \mathrm{~ns}$ pulses at 1.5 GI:? with a pulse repetition period of $600 \mathrm{~ns}$. Based on these time delay spread and attenuation measurements they proposed a statistical model of the indoor radio channel. By sweeping the RF frequency over $200 \mathrm{MHz}$ instead of keeping it fixed, they were able to get a $5 \mathrm{~ns}$ resolution. The distance-power law obtained varied from 1.5 in hallways to 3 in offices. There was one occurrence of a value over 4 in one large open machine shop. The median value for the rms time delay spread was $25 \mathrm{~ns}$ with a maximum of about $50 \mathrm{~ns}$. For these calculations 
they limited themselves to pulse responses with less than $100 \mathrm{~dB}$ of total path loss. The lower pulse responses were discarded. No hallways measurements were used: these situations had line-of-sight rays plus strong rays at long delays. Line-of-sight situations do not really need a model to predict performance: the reception should be very good. Saleh and Valenzuela's ms time delay spread values were much smaller than those obtained by Devasirvatham [27] in the same building. His data included signals of low power level (100-140 $\mathrm{dB}$ path loss). Taking a subset of this data wher path loss was less than $100 \mathrm{~dB}$, his results were similar.

In the proposed model, the rays of the received signals arrive in clusters. The rays' amplitudes are independent Rayleigh random variables. Their variances decay exponentially with cluster delay as well as with ray delay within a cluster. The phase angles are uniformly distributed. The clusters and the rays within a cluster form Poisson arrival processes with different but fixed rates. The cluster formation deperids on building structure. The rays in a cluster are formed by multiple reflections from objects close to the transmitter and receiver.

Bultitude [17] made some CW envelope, phase and attenuation measurements in two types of building. The first one was partitioned in small offices and the second was an open concept building. Temporal fading measurement where the transmitter and the receiver are static, were taken for long periods of time. In the first building the fading intervals were 20 to 40 seconds long with periods of almost constant envelope level in between them. In the second type, the fading intervals were less well defined in time: there was not really any period of constant envelope level between fading intervals. The fading dynamic range was $30 \mathrm{~dB}$ in the first building and $17 \mathrm{~dB}$ in the second. Both buildings showed wide phase variations.

Cumulative distribution functions of the received signal envelope were computed for the fading intervals only. They followed fairly well a Rician distribution with $\mathrm{K}=$ $-6.8 \mathrm{~dB}$ ( $\mathrm{K}=$ random power component/specular power component) in the first building. For the second building, a Rician distrioution with $K=-11 \mathrm{~dB}$ was a good fit to the experimental curves. Experimental and simulation work directed by Bultitude, reported 
in internal. reports, showed that the spatial distribution of the received signal with mean variation removed was also Rician with $K=-2 \mathrm{~dB}$ when the physical environment in the building was static. He also calculated time autocorrelation functions for some fading intervals. They showed that the channel statistics stayed approximately the same for time periods of up to 4 seconds.

Rappaport and McGillem reported narrowband and wideband measurements in 5 factories [4], [5]. Attenuation measurements showed a distance-power law of 1.5 to 2.8 depending on factory topography. For narrowband measurements the cumulative distribution function of the large scale area signal level were well described by a lognormal distribution. Small scale fading range was typically 30-35 dB. The distributions of small scale envelope fading were of three types. For 17 out of 26 line-of-sight with light clutter (one of four categories of building used by the authors) measurement locations, the distribution was Rayleigh or Rice with $K<2 \mathrm{~dB}$ (K is defined here as the ratio specular power/random power, the opposite of Bultitude in [17]). The other 9 were distinctly Rician. At 3 out of 8 obstructed with light clutter locations and at 9 of 15 obstructed with heavy clutter locations, the curve fitted a log-normal distribution. The curve produced using all the data combined ( 20000 signal measurements) followed a log-normal distribution below the median value and a Rayleigh distribution above the median value. In temporal fading measurements, the curves were in good agreement with a Rician distribution with $\mathrm{K}=10 \mathrm{~dB}$. Fading dynamic range was less than $20 \mathrm{~dB}$.

Wideband measurements taken in the factories were reported by Rappaport in [6]. Overall attenuation measurements gave a power-distance law (2.16) very close to the narrowband measurements. Rms time delay spreads were found to be uncorrelated to the transmitter-receiver distance or the factories topography. They seemed to depend more on the building age, inventory, wall location and ceiling height. The median time delay spread was 96 ns over line-of-sight (LOS) paths and 105 ns over obstructed paths. These time delay spreads are on the order of values reported by Devasirvatham in [11].

Lee and Yeh [28] in the seventies and more recently Cox, Murray, Amold, Noris and Wazowicz [13] took measurements to collect data on vertically and horizo.ttally polarized 
waves and cross-polarization coupling in the $800 \mathrm{MHz}$ range. Cross-polarization coupling is defined in [13] as

$$
20 \log \frac{E_{x}}{E_{t}}
$$

where $E_{x}$ is the average field amplitude of the orthogonal polanization and $E_{t}$ is the average field amplitude of the transmitted polarization. Although for the mobile radio channel, the results of [28] can be applied to the indoor channel. Both types of polarization were observed to have Rayleigh distributed envelope levels and low correlation $(<.2)$ for any antenna spacing. Their local means were within $3 \mathrm{~dB}$ of each other. Cross-polarization coupling measurements done $\mathrm{cn}$ the mobile to base path showed that the orthogonal polarized signal was about $6 \mathrm{~dB}$ lower than the original polarization.

Cox, Murray et al took cross-polarization coupling measurements inside two laboratories buildings (same building as in [27]) and inside and outside of eight houses with vertical and horizontal dipoles. As mentioned earlier in section 1.3, a horizontal dipole has two nulls in its radiation pattern. Under the assumption of a uniform azimuthal distribution of horizontally polarized components, the power reading from a horizontal dipole would be $3 \mathrm{~dB}$ less than the actual value. Under this assumption however, the effect of the twc nulls takes less importance: the energy density is the same for all direction in the horizontal plane, so the orientation of the dipole matters less. They werc able to crudely verify the validity of the assumption by comparing end-on (antenna pointing towards the transmitter) and broadside (antenna being $90^{\circ}$ away from pointing tow Is the transmitter) signal medians. For a uniform azimuthal distribution, median values should be close together.

Using the dipole for measurements, the definition of cross-polarization coupling now becomes

$$
\frac{\left(L_{h(\text { broadsile })}+L_{h(\text { cnd-on })}\right)}{2}+3-L_{v}
$$

$L_{h}$ and $L_{v}$ are the horizontally and the vertically polarized components. The mid-point between the broadside and end-on value was used as the horizontal cornponent. A 
cross-polarization coupling value of $0 \mathrm{~dB}$ indicates that the power is equally distributed between the horizontally and the vertically polarized components. A negative value means that the vertically polarized component is stronger. These results will be reliable only if horizontal plane wave propagation is assumed. With their dipole measurements, they cannot calculate the correction for vertical angle of arrival. Random angle of arrival and random polarization would give a cross-polarization coupling value of +3 dB. This led them to believe that positive values could mean that wave propagation is not restricted to the horizontal plane.

In the two buildings the uniform azimuthal distribution assumption was valid. The cross-polarization coupling median was $.9 \mathrm{~dB}$ and $1.8 \mathrm{~dB}$ for the small and the large building respectively which neans that significant propagation occurred outside the horizontal plane. The measurements made inside and outside the houses gave median cross-polarization coupling of $-2.5 \mathrm{~dB}$ and $-3.5 \mathrm{~dB}$ respectively. The lower value for outside was due $t$ : these locations having a more direct path to the transmitter: there were less scatterers to de-polarize the vertically polarized transmitted signal. Crosspolarization coupling measurements were also taken in [23] and were all greater than $-10 \mathrm{~dB}$ with most above $-6 \mathrm{~dB}$.

The radiating elements discussed so far were only single antenna. They are widely used because they are easy to deal with: quick installation and removal, low cost to buy or build and their radiating characteristics are well known. Distributed antennas are another method that could be used [29]. Slotted coaxial cables, also known as leaky feeders, are another option to use in an indoor radio communication system. Motley and Palmer [4] and Reed and Stevenson [30] compared the propagation characteristics of antennas, slotted coax and distributed antennas. They concluded that slotted coaxial cables give better signal coverage and lower floor to floor penetration than a single antenna. Motley and Palmer established the performance cross-over point of the slotted coax versus single antenna at $20 \mathrm{~m}$. In a building with large open areas $(>29 \mathrm{~m})$, single antennas would give a better performance but for a building with many hallways and closed rooms the slotted coax would be the better choice. 


\subsection{Review of previous experiments}

This section will review some installation details of experiments mentioned in the previous section. This was done to help design our experimental installations (detailed in chapter 3). The main points we were looking for in these experiments were: the data sampling rate, the type of data acquisition system, the type of antenna used, the type of detection/demodulation, the parameters measured, the general design of the experiment.

Hoffman and Cox [22] used a comer reflector at the transmitter and a vertical $\lambda / 2$ skirted coaxial antenna at the receiver. The receiver was a spectrm analyzer connected to an $x$-y plotter. The receiver antenna was scanned horizontally in $\mathbf{4}$ feet square areas at three different heights. Paper charts produced by the plotter were digitized every .1 $\lambda$ and stored on computer for processing. All recordings were scaled in $\mathrm{dB}$ relative to a point just outside of the building.

Cox, Murray and Norris [23] were using a modified walkie-talkie transmitting at $815 \mathrm{MHz}$ with a power output of .8 Watt. It had a $\lambda / 2$ coaxial sleeve dipole un top of the unit. The unit was hand-held at arm's length 4.5 feet above the floor and scanned along a certain pattern for about 20 seconds. The receiving antenna was a $815 \mathrm{MHz}$ collinear array consisting of 4 dipole elements mounted vertically on top of a 27 feet mast. The receiver was a modified commercial FM communication unit: an $11.7 \mathrm{MHz}$ intermediate frequency (IF) was extracted before the limiter, down-converted to $13 \mathrm{kHz}$, bandpass filtered ( $3 \mathrm{~dB} b w=8 \mathrm{kHz}$ ) and linearly detected. It had a $45 \mathrm{~dB}$ measurement range. Since the transmitter power was fixed, a step attenuator was used to control the RF input signal level. The data acquisition system consisted of a 12 bit resolution digital storage oscilloscope set to record 2048 samples in the 20 seconds period $(\approx 100$ samples/second). After collection, the data was scaled to convert recorded voltage to dis relative to signal level at a reference location (transmitter 14.2 feet away from the receiver). The scaling took into account the reference location voltage level, the DC offset and the attenuator setting. Scaled data was stored on a desk-top computer. For the cross-polarization coupling measurements, the transmitter was held horizontally and scanned once with the antenna pointing toward the receiver and a second time broadside 
to it. These two values were then averaged to get a horizontal power component. Low values of cross-polarization coupling could not be measured accurately due to the uncertainty of the measurement technique: the horizontal antenna was held within $10^{\circ}$ of the horizontal plane. This misalignment would, by itself, produce a residual cross-polarization of $-15 \mathrm{~dB}$ [13].

The method used by Alexander [24] was designed to provide data on signal strength distribution on a per-room basis, withnut the need for a precise iocation reporting system. A small hand-held transmitter was used as a mobile source. It was moved around each room, : $\mathrm{m}$ above the floor, at a slow walking pace to cover as much area as practically possible. The transmitter had a power output of $30 \mathrm{~mW}$ and was using a vertically polarized $\lambda / 4$ monopole with ground plane. The receiver was stationary and consisted of a $\mathrm{CW}$ receiver (dynamic range $60 \mathrm{~dB}, \mathrm{bw}=120 \mathrm{kHz}$ ) linked to a computer via an 8 bit analog-to-digital converter. The receive antenna was a vertically polarized $\lambda / 2$ dipole, $1.2 \mathrm{~m}$ above the floor. Each room was surveyed for 1 minute, with about $10^{6}$ samples taken (sampling rate $\approx 16500$ samples/second).

Devasirvatham used a $40 \mathrm{Mbits} / \mathrm{s}$ pseudo-noise sequence to modulate (BPSK) a 850 $\mathrm{MHz}$ carrier in the transmitter (as used by Cox in the mobile radio environment [31]). The receiver used the same sequence but running $4 \mathrm{kbit} / \mathrm{s}$ slower. As this sequence swept past the time smeared received signal, it would produce correlation peaks whenever it became in phase with the transmitted signal (4000 times per second), thus producing the power-delay profile of the received signal. The receiver output was sampled by a computer-based data acquisition system every $10 \mu \mathrm{s}$, an effective sampling interval of $1 \mathrm{~ns}$ [31]. The first 2048 points per recording were stored. The transmitter and receiver antennas were both vertically polarized sleeve dipoles. The transmitter astenna was 1.3 $\mathrm{m}$ above the floor. The receiver antenna was on top of a 27 feet mast [11] for indoor to outdoor measurements and $1.8 \mathrm{~m}$ above the floor for indoor to indoor measurements [27]. For each transmitter and receiver location, 8 profiles were recorded with the receiver or transmitter placed on 8 positions on the perimeter of a $1.2 \mathrm{~m}$ square. All recordings were referenced to the power level obtained when the receiver was.. $\mathrm{m}$ away. The 
8 profiles were averaged point by point to form one profile for which the root mean square (rms) time delay spread was calculated.

Bultitude [17] used a synthesizer at $910 \mathrm{MHz}$ and an amplifier connected to a $\lambda / 4$ monopole with ground plane as his transmitter. The receiver, a commercial telemetry receiver, down-converted the signal to an IF of $21.4 \mathrm{MHz}$. This signal was then downconverted to baseband in a quadrature double-balanced mixer circuit. The in-phase and quadrature components were sampled by a 12 bit analog-to-digital converter then stored in a micro-computer. The receiver automatic gain control (AC.C) output was also recorded. This output gave a reading on the signal strength to do attenuation measurements. Each synthesizer in the system (receiver and transmitter) was slaved to a single rubidium frequency standard to maintain phase accuracy. The data sampling rate was 10 samples/second.

The transmitter used by Rappaport and McGillem [5] consisted of a pulse generator modulating a $\mathrm{CW}$ signal at $1282 \mathrm{MHz}$. The mixer output was amplified and fed to a discone antenna through a decade attenuator for power control. The receiver was configured to do attenuation and pulse measurements without reconfiguration. The received signal was amplified and passed through a $15 \mathrm{~dB}$ directional coupler. One output was sent to an envelope detector while the other was fed to a modified commercial receiver whose AGC was inactivated. The DC level of the receiver's last IF stage was proportional to the $\mathrm{CW}$ signal level. A digitizing oscilloscope recorded the detector's output. These recordings were sent to a computer via an HP-IB connection. Path loss, small-scale fading, temporal fading and shadowing measurements were made. The small-scale fading measurements were done by moving the receiver cart along a 1.2 $m$ track, recording 128 IF voltage values in 5 seconds. For each receiver location, 3 runs were made on two paths 1 to $3 \mathrm{~m}$ apart. On the first track, two runs were made with antenna height of $2 \mathrm{~m}$ and $1.5 \mathrm{~m}$. The third run was made on the second track with an antenna height of $2 \mathrm{~m}$. Temporal fading measurements (receiver and transmitter stationary) were made in areas of significant channel motion. They were made in 100 seconds run and data was recorded at 10.24 samples/second. The dynamic range of the 
envelope measurements was limited to the linear scale of the oscilloscope, about $25 \mathrm{~dB}$. The measurement range of the system was $90 \mathrm{~dB}$.

\subsection{Experiment outline}

Our experimental program is aimed at characterizing the indoor channel using frequency, space and polarization diversity for narrowband communications system. Certain parameters are needed tn effectively characterize the channel for diversity. These parameters can be obtained by doing wideband or narrowband measurements. Wideband measurements are more delicate to perform and involve a very large amount of data. The interpretation of this data and the extraction of the parameter needed is always more difficult than vith narrowband measurements. Narrowband communications system do not require any phase measurements because for bandwidth $<200 \mathrm{kHz}$, the information on the phase is not critical to the performance of the system. We will be using tones (very narrow bandwidth) in our experiment and will not make any provision to record any phase information.

The frequency spacing (in frequency diversity) will be within a $25 \mathrm{MHz}$ band $(903$ to $928 \mathrm{MHz}$ ) which is allocated to indoor radio communications. In the space diversity experiment, the antenna separations will remain within a reasonable distance. To keep the conduct of the experiment within a reasonable time frame and to keep the quantity of data to a manageable level, five frequency separations and five antenna separations will be sadied.

To cover the frequency band evenly, the following frequency separations will be selected: $1,5,10,15$ and $20 \mathrm{MHz}$. The maximum distance between antennas is limited to $2 \lambda$. To avoid the risk of having the two receive antennas coincide with two signal nulls simultaneously, the separations will be: $.75 \lambda, 1 \lambda, 1.5 \lambda, 1.66 \lambda$ and $2 \lambda$.

Signal combining will not be implemented in hardware to keep the receiver hardware simple and to allow flexibility in the choice of combining method. A software algorithm will implement the combining using the recorded data as input. By simply changing the algorithm, many combining methods can be tried. Selection combining was chosen 
for analysis purposes because of its good performance and its relative simplicity and practicality.

The experiment area should be as large as possible to allow a good range of LOS and non-LOS locations to be studied. Power limitation of the transmitter will probably be a limiting factor in the actual area covered. Recordings at a large number of locations will improve the statistical validity of our measurements. This will have to be balanced against available storage for the data collected and against processing time. As reported in many of the experiments described in the previous section, floor to floor penetration is poor, therefore our area will be limited to locations on the same floor.

Power levels, attenuation readings and calibration data will be recorded during the measurements to present some results on the attenuation characteristics of the building. 


\section{Chapter 3 \\ Description of experimental installations and procedures}

\subsection{Introduction}

The design and building of the experimental installations was a critical part of the experiment. The calibration of the installations and the actual measurement procedure were also an important aspect. This chapter will describe the details of each.

Before finalizing the design of the transmitter and receiver, we felt that some preliminary measurements were needed to get additional knowledge on the channel and the attenuation of the building and to get some practical experience with the equipment. A transmitter and receiver were built to conduct initial measurements. The information obtained from these initial measurements was used to refine our final design.

Although studying three different types of diversi $1 ;$, there were only two basic configurations for the transmitters and receivers: one for frequency diversity and the other for space and polarization diversity. For these two types, the transmitter remains the same and the receivers differed only in the position and orientation of the second antenna. In all cases the experimental installation can be broken down into four building blocks: transmitter, receiver, antenna and data acquisition. They will be studied in this sequence. The data acquisition block was further divided in two sections for clarity of 
presentation: a section on the analog-to-digital converter and a section on the Vectra personal computer.

To extract meaningful information from the data produced by the analog-to-digital converter, some scaling had to be done. To achieve this, the system had to be calibrated. The calibration procedure and its results will be presented. Finally, the detailed measurement procedure used to collect the daia will be outlined.

The preliminary measurements will be discussed in the next section. Section 3.3, 3.4, 3.5 and 3.6 will cover the transmitters and the receivers. The antenna system will be discussed in section 3.7. The analog-to-digital converter and the Vectra PC will be discussed in section 3.8 and 3.9. The chapter will conclude with a description of the calibration steps and the measurement procedure.

\subsection{Preliminary measurements installation}

\subsubsection{Transmitter and Receiver.}

The preliminary measurements were done only for frequency diversity. The transmitter design used here was very similar to the one used for the final measurements. Figure 3.1 shows the block diagram for the transmitter. A $915 \mathrm{MHz}$ local oscillator signal was fed to a double balanced mixer. The IF (intermediary frequency) signal was provided to the mixer at five different frequencies: $.5,2.5,5.0,7.5$ and $10 \mathrm{MHz}$. The mixer output was two equal amplitude tones equally spaced (by the IF frequency value) above and below the $915 \mathrm{MHz}$ carrier. This cutput was amplified and fed to a simple antenna consisting of a $10 \mathrm{~cm}$ monopole conneited to a female SMA adapter. The transmitter remained static in one room. 


\section{HP 8656B}

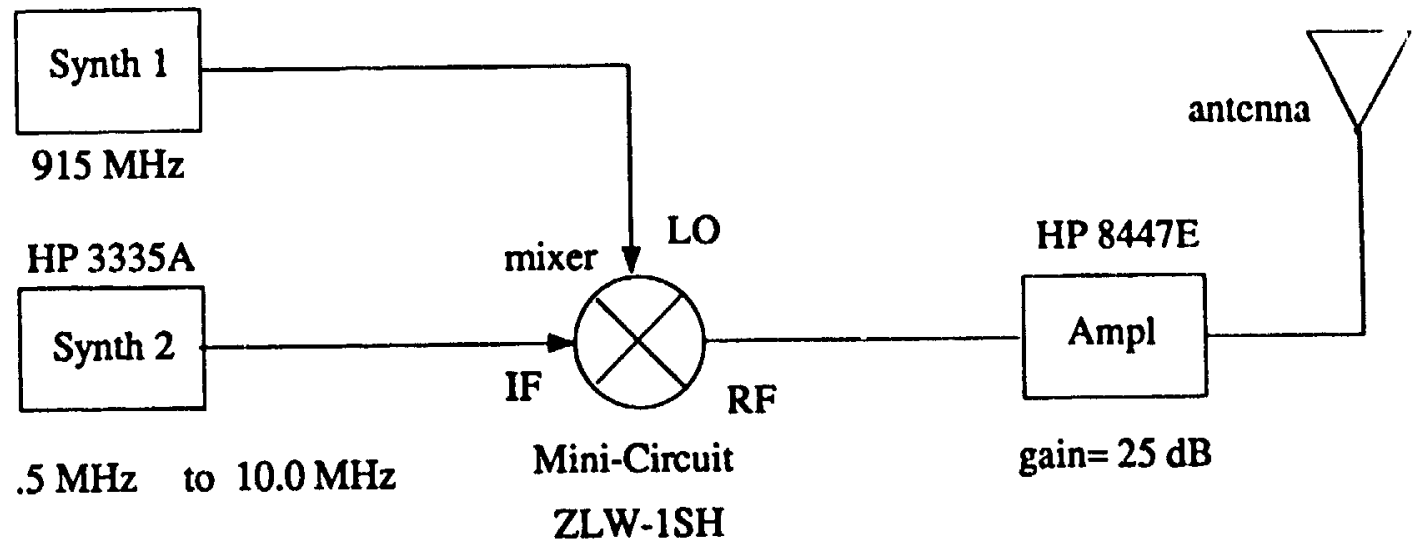

Figure- 3.1: Block diagram of preliminary transmitter

The receiver was based on a Hewlett-Packard $8590 \mathrm{~A}$ spectrum analyzer. Figure 3.2 shows the block diagram for this receiver. The signal from the receive antenna was amplified and then fed to the spectrum analyzer. Through the Hewlett Packard Interface Bus (HP-IB) a HP Vectra PC controlled the functions of the analyzer and recorded the power level of each tone. The receiver was installed on a wheeled cart so it could be moved to various locations.

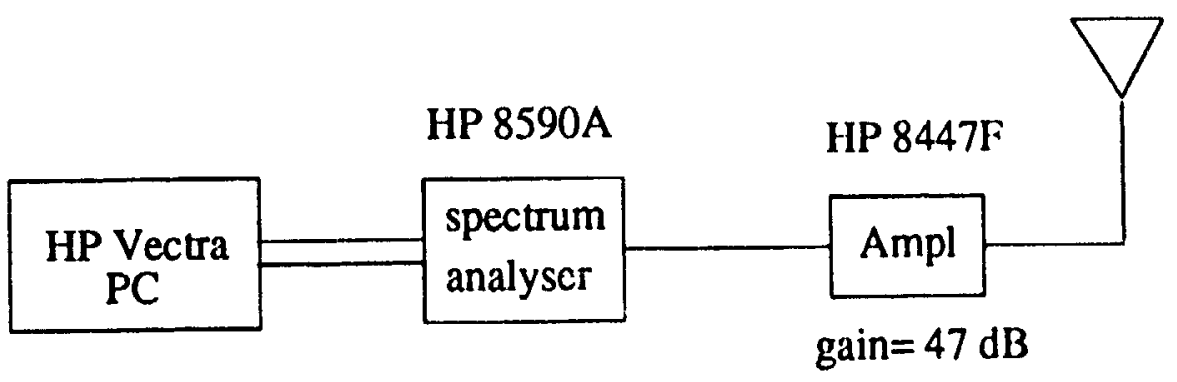

Figure- 3.2: Block diagram of preliminary receiver 


\subsubsection{Lessons learned}

Tiiese measurements were aimed at getting additional knowledge on the channel to help with the final design of the transmitter and receiver and to become familiar with the equipment and its limitations. The spectrum analyzer, with its variable frequensy span and large dynamic range, was a good instrument to use in preliminary measurements but it had drawbacks which ruled out its use for more precise measurements.

With the fastest program loop possible, the PC could take one pair of readings per $420 \mathrm{~ms}(\approx 2.3 \mathrm{samples} / \mathrm{second})$. This was not fast enough to acquire fast fading events. The analyzer's fastest sweep time was $20 \mathrm{~ms}$ : this meant there was about 15 to $18 \mathrm{~ms}$ between the sampling of the first and the second tone. This does not seem like much but ideally each tone should be sampled simultaneously to see how much they differ at a given instant.

The need for some kind of spatial averaging quickly became evident. With the transmitter and the receiver antenna staying static during recordings, the resulting signal amplitudes would not show much variation. One signal was consistently much higher than the other. Some measurements were made with the receiving antenna being moved by hand in a circular fashion. The sajority of the fading events then recorded spanned a dynamic range of about $40 \mathrm{~dB}$. These measurements gave a good indication of the output power needed to cover the area selected for the final measurements.

Some other practical facts became evident during these measurements: SMA rigid or semi-rigid coaxial cables are better suited for use in the $900 \mathrm{MHz}$ frequency range than BNC coaxial cables. They have much better signal attenuation characteristics.

\subsection{Frequency diversity transmitter}

In its final version, the frequency diversity transmitter was not very different from the one used in the preliminary measurements. Figure 3.3 is a block diagram and plate 1 is a photograph of the transmitter. The two frequencies are produced by the same method: a lower frequency F $(.5,2,5.0,7.5$ and $10 \mathrm{MHz})$ is mixed with a carrier to 


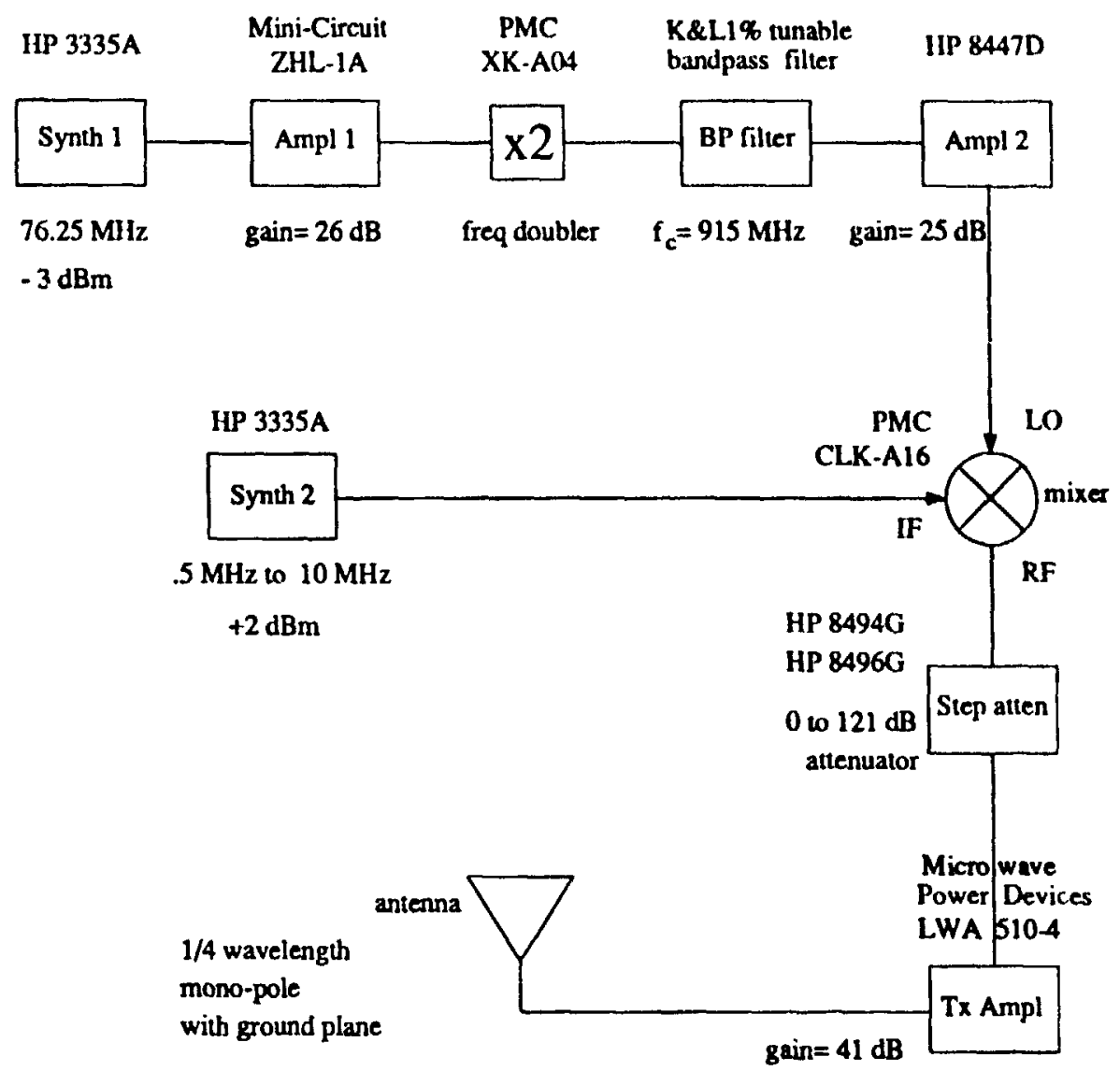

Figure- 3.3: Block diagram Frequency diversity transmitter

produce two tones spaced by $\mathrm{F}(1,5,10,15$ and $20 \mathrm{MHz})$, centered around $915 \mathrm{MHz}$. The actual output frequencies were 914.5 and $915.5 \mathrm{MHz}, 912.5$ and $917.5 \mathrm{MHz}, 910$ and $920 \mathrm{MHz}, 907.5$ and $922.5,905$ and $925 \mathrm{MHz}$. The output of the mixer was fed to an amplifier through a two stage step attenuator. It provided attenuation in $1 \mathrm{~dB}$ steps from 0 to $121 \mathrm{~dB}$. By changing the attenuation at the transmitter, the average power at the receiver could be adjusted to be in the dynamic range of the receiver, no matter where the transmitter was located.

The transmitter power amplifier was in two stages and had a total gain of $41 \mathrm{~dB}$. Two heat sinks were installed on the power amplifier to avoid significant changes in the amplifier gain. From the amplifier, the signal was sent to the transinit antenna 1.6 
$m$ above the floor. The maximum output power of the transmitter (attenuator at $0 \mathrm{~dB}$ ) is listed in table 3.1 .

\begin{tabular}{|c|c|c|c|c|c|}
\hline \multicolumn{6}{|c|}{ OUTPUT POWER } \\
\hline Spacing & $1 \mathrm{MHz}$ & $5 \mathrm{MHz}$ & $\begin{array}{c}10 \\
\mathrm{MHz}\end{array}$ & $\begin{array}{c}15 \\
\mathrm{MHz}\end{array}$ & $\begin{array}{c}20 \\
\mathbf{M H z}\end{array}$ \\
\hline $\begin{array}{c}\text { Power } \\
(\mathrm{dBm})\end{array}$ & 30.4 & 31.0 & 30.3 & 30.5 & 31.0 \\
\hline
\end{tabular}

Table 3.1 Transmilter maximum output power

The upper part of figure 3.3 shows how the $915 \mathrm{MHz}$ carrier was produced with a low frequency synthesizer. A $76.25 \mathrm{MHz}$ tone (from synth 1) was fed to a $26 \mathrm{~dB}$ gain amplifier (amp 1) then to a frequency doubler. Many harmonics of the $76.25 \mathrm{MHz}$ tone were produced at the output of the amplifier. This output was fed to the frequency doubler. At the output of the doubler, all the harmonics, except the one at $915 \mathrm{MHz}$, were filtered out with a K\&L tunable band-pass filter. At the amplifier's output, the lower harmonics were much stronger than the higher ones. Better results were achieved by passing the $457.5 \mathrm{MHz}$ tone (6 $6^{\text {th }}$ harmonics) through a doubler and amplify it rather than trying to directly use the tone at $915 \mathrm{MHz}$ (12 $12^{\text {th }}$ harmonics). This carrier was then amplified to $+6 \mathrm{dBm}$ and fed to the mixer. The carrier produced this way was as accurate in frequency as the initial synthesizer $( \pm 10 \mathrm{~Hz})$.

The fiequency spectrum (for each frequency spacing) at the transmitter output is shown in figure 3.4 to 3.8 . It would not have been practical to try removing the sidebands at the transmitter output. In order to pass the desired tones, the required filter bandwidth would have been so large that many sidebands would get through and the filter would be of minimal use. Since the intermodulation products are much weaker $(<-20 \mathrm{~dB})$ than the desired tones, no attempt was made to remove them. The carrier was also low enough that it did not interfere in the measurements. 


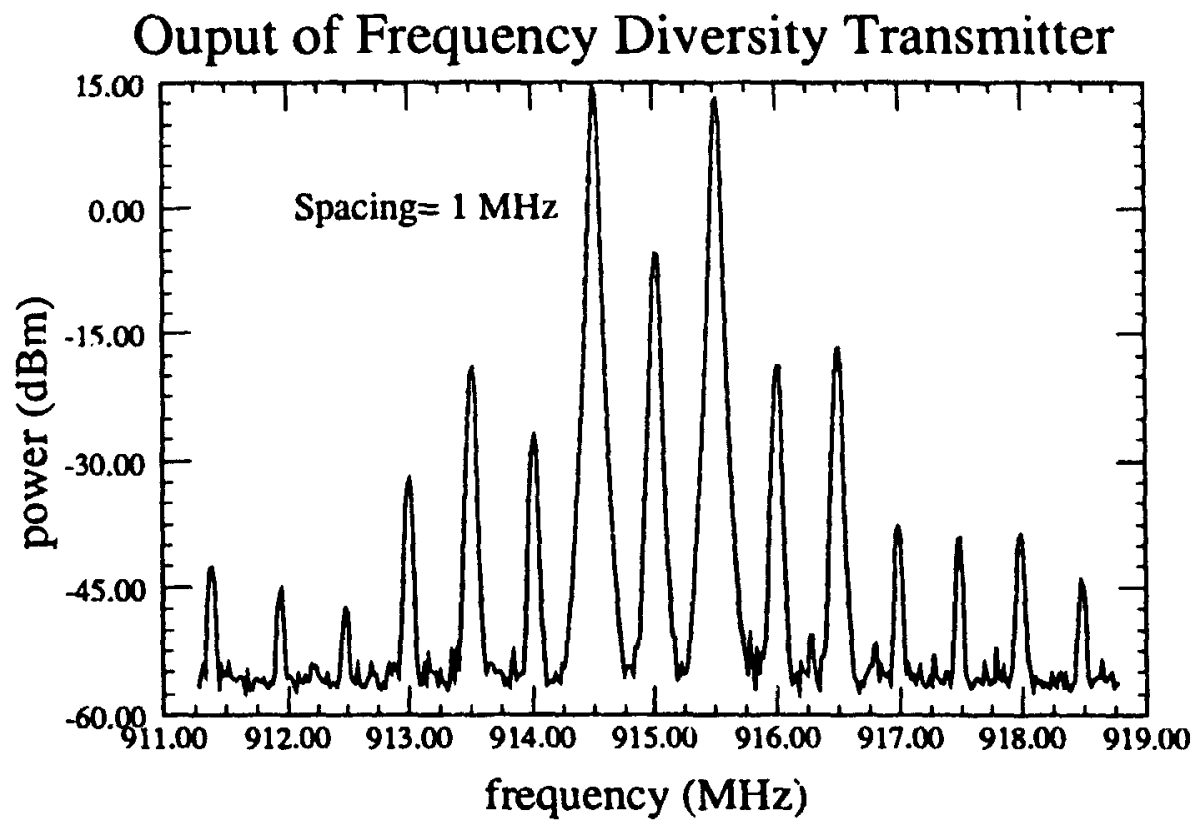

Figure- 3.4: Power spectrum of frequency diversity transmilter output, I MItz spacing

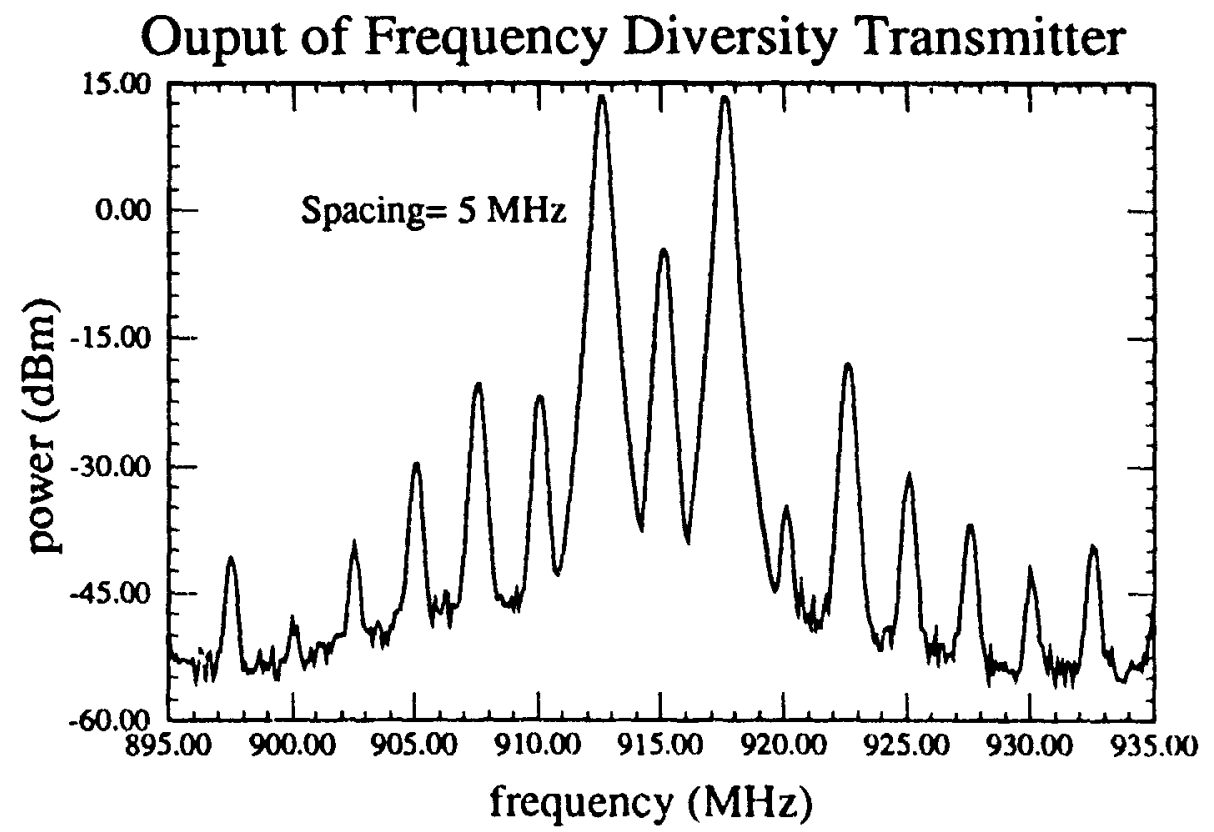

Figure- 3.5: Power spectrum of frequency diversity transmilter oulpul, $5 \mathrm{M} / 1 \mathrm{z}$ spacing 


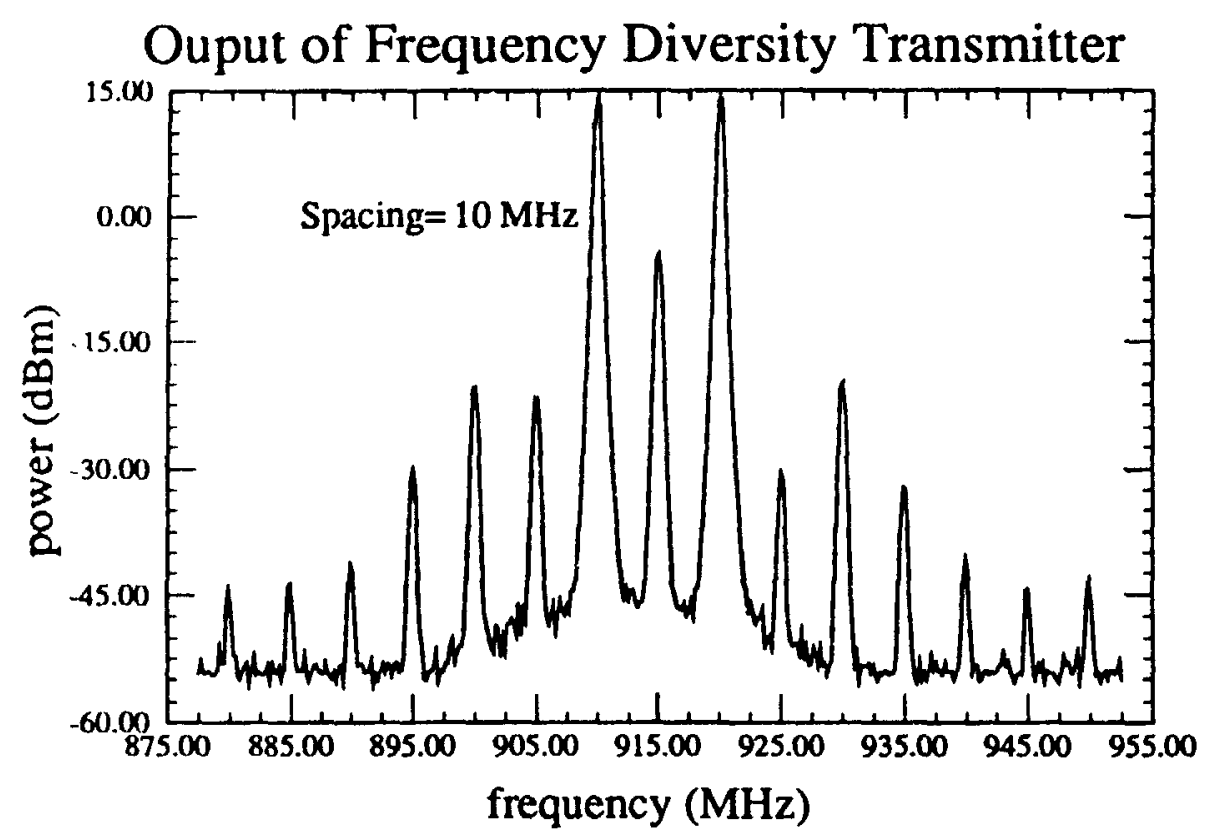

Figure- 3.6: Power spectrum of frequency diversity transmitter output, $10 \mathrm{MHz}$ spacing

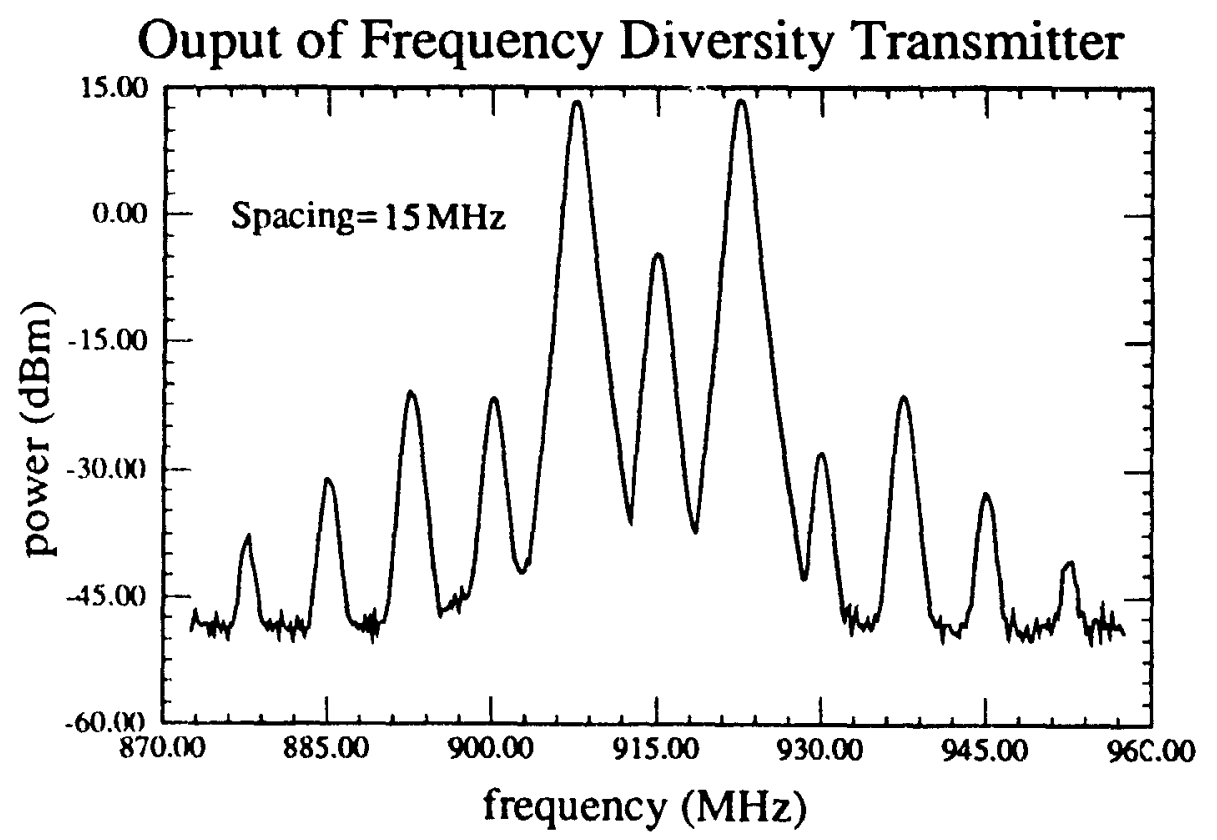

Higu. e- 3.7: Power spectrum of frequency diversity transmilter ouput, 15 MHI2 spacing 


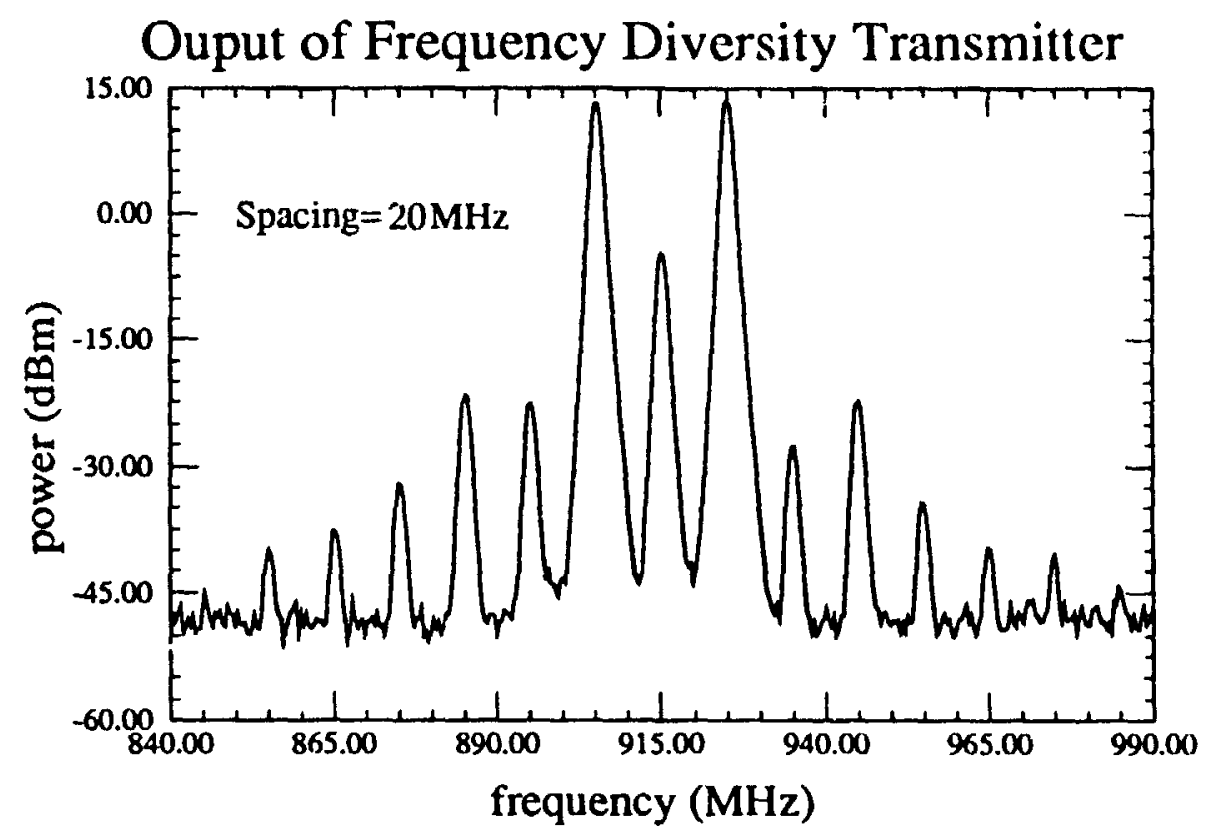

Figure- 3.8: Power spectrum of frequency diversity transmitter ouput, $20 \mathrm{MH} / \mathrm{z}$ spacing

\subsection{Space and polarization diversity transmitter}

This transmitter was very similar to that used for frequency diversity. Figurc 3.9 shows a block diagram of the transmitter. Only one frequency was required so the mixer was removed and the carrier was fed directly to the step attenuator. To compensate for the absence of the mixer's insertion loss, a $10 \mathrm{~dB}$ coaxial attenuator was added after the step attenuator. The maximum output power of this transmitter was $33.8 \mathrm{dBm}$. 


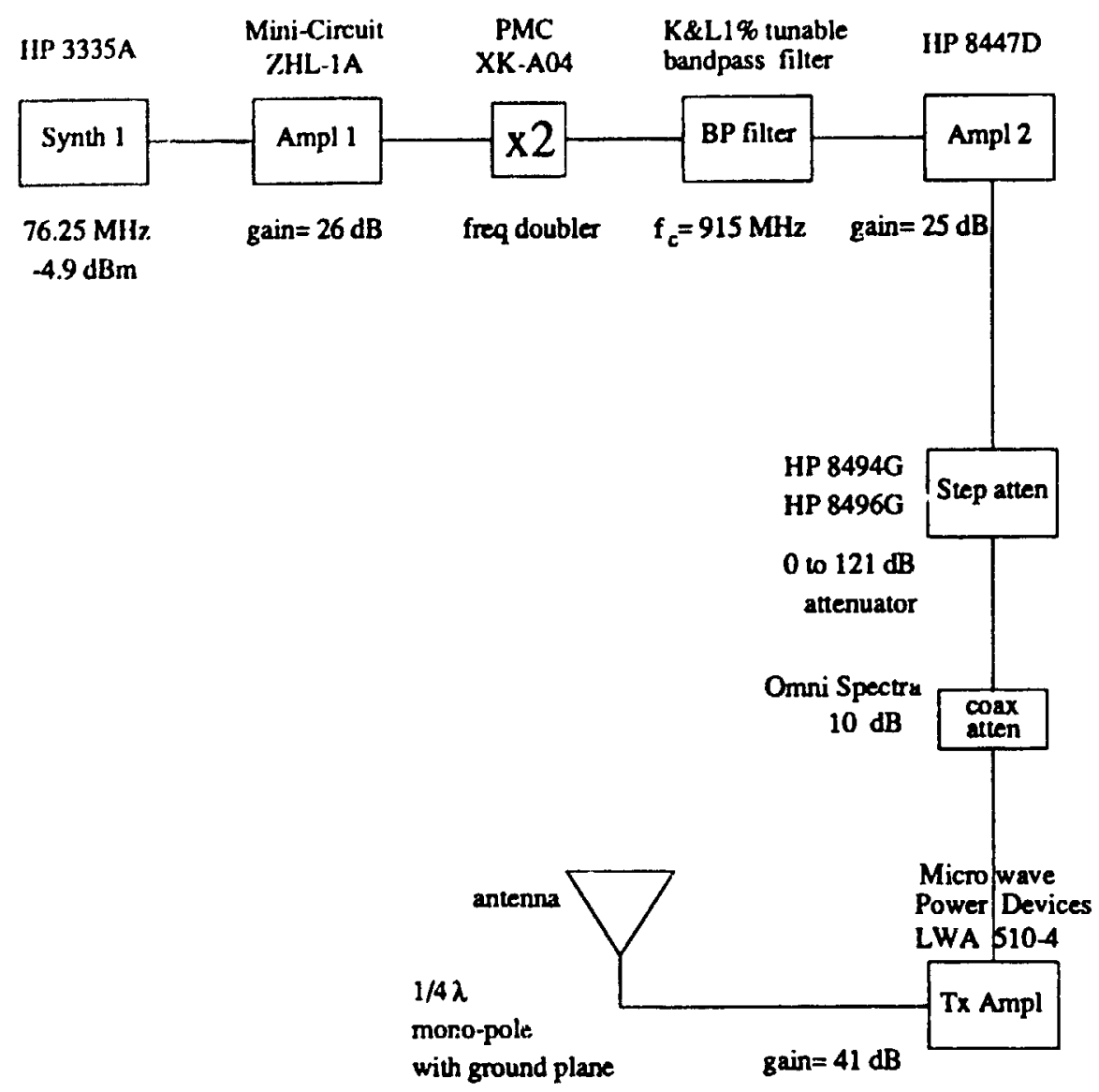

Figure- 3.9: Block diagram of the spacelpolarization diversity transmitter

\subsection{Frequency diversity receiver}

The receiver can be broken down in three main parts: the front end, the IF part and the demodulation part. Figure 3.10 shows a block diagram of the receiver and plate 2 shows a photograph of it.

The receiver was set on a wooden cart. The antenna was on a cardboard frame resting on top of the two synthesizers, about $1.75 \mathrm{~m}$ above the floor. The front end part consisted of the antenna, a preamplifier, an amplifier and a power divider. The antenna was of the same type as the transmit antenna and will be covered in more detail in section 3.7. The preamplifier and amplifier are combined in one module, the HP 


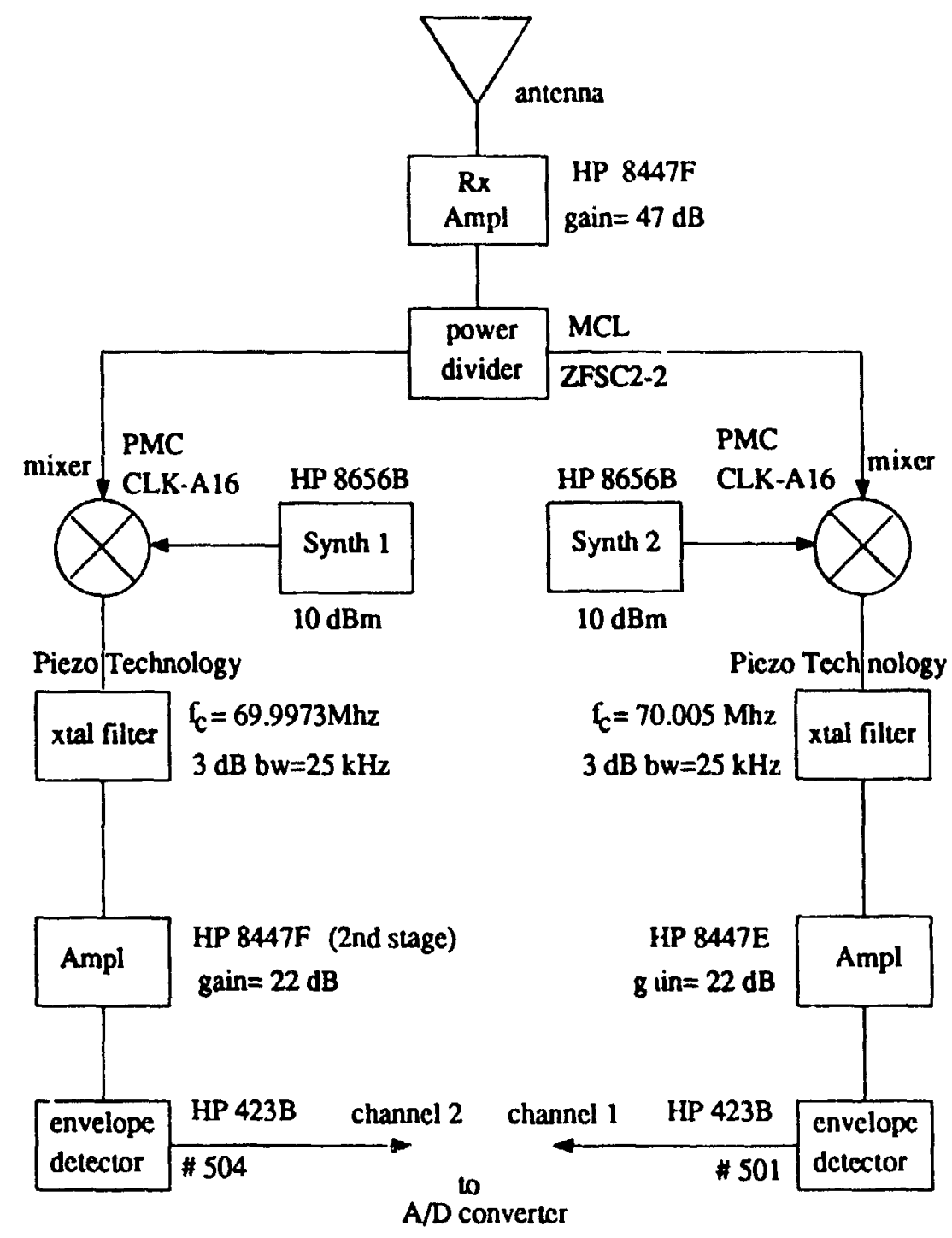

Figure- 3.10: Block diagram of frequency diversity receiver

$8447 \mathrm{~F}$, for a total gain of $47 \mathrm{~dB}$. The power divider was balanced to $\pm .1 \mathrm{~dB}$ with an insertion loss of $3.1 \mathrm{~dB}$.

The IF portion of the receiver had two identical branches in parallel. Each branch consisted of a mixer, fed from the power divider, an HP 8656B signal synthesizer to drive the mixer, a bandpass crystal filter and an amplifier to boost the down-converted signal. The nominal center frequency of the two filters was set as $70 \mathrm{MHz}$. The 
exact frequency was confirmed by slowly sweeping a signal at the input and noting the frequency where the power output was maximum. The center frequencies of the two filters were off from each other by about $3 \mathrm{kHz}$ and their insertion loss by $.6 \mathrm{~dB}(2.1$ and $2.7 \mathrm{~dB}$ ). The transfer function of the filters, as shown in figure 3.11 and 3.12 is good in the region around the center frequency. It has spurious responses a few hundreds of $\mathrm{kHz}$ away which is characteristic of crystal filters.

The synthesizer frequency for each branch was set to down-convert the lower and higher tone to the center frequency of its respective filter. The power spectrum at the output of each filter is shown in figure 3.13 and 3.14 for a spacing of $1 \mathrm{MHz}$. We can see that the undesired tone and the carrier were not completely blocked out but they were weak enough $(<-40 \mathrm{~dB}$ and $<-55 \mathrm{~dB}$ ) that their effect was negligible. The situation for the other spacings is similar but the undesired tone and the carrier were even weaker.

The demodulation portion was straightforward. We were interested only in the envelope and not in the phase of the signal. The demodulator used a crystal detector specifically matched to a $50 \Omega$ load resistor. In addition, the two detectors were a matched pair to ensure that their response would be as close as possible to each other. The crystal detectors and the $50 \Omega$ resistors produced a voltage proportional to the envelope of the input signal. The data acquisition system was connected to these resistors. 
Filter frequency response

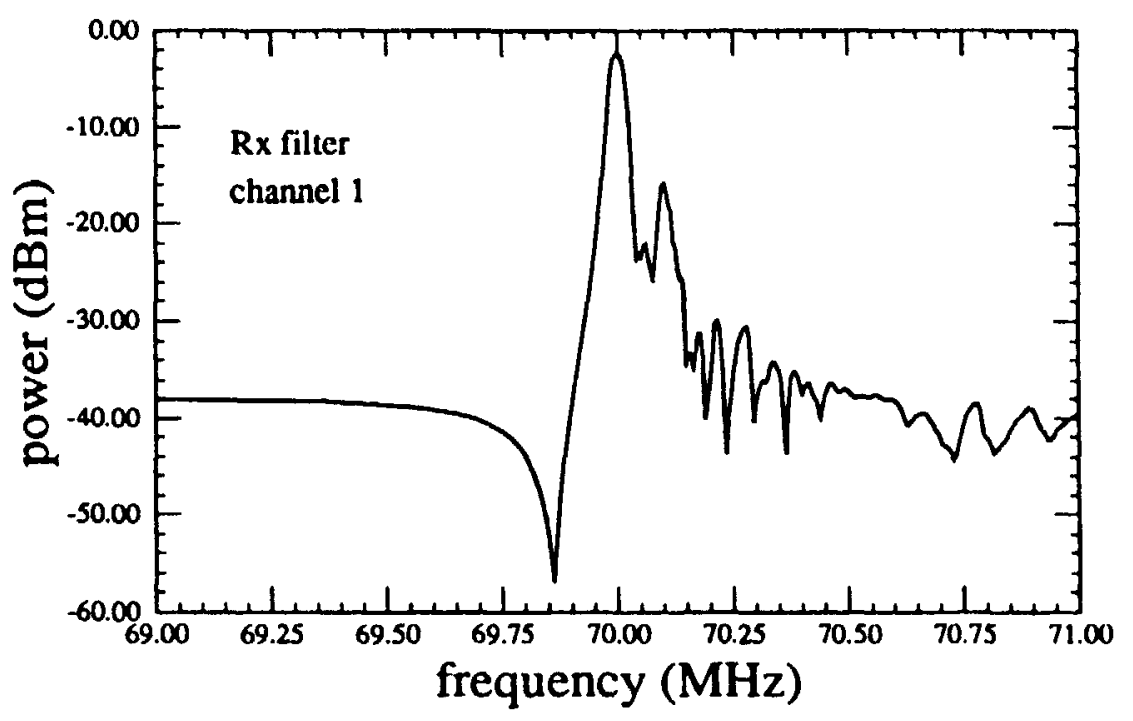

Figure- 3.11: Transfer function of receive crystal filler channel 1

Filter frequency response

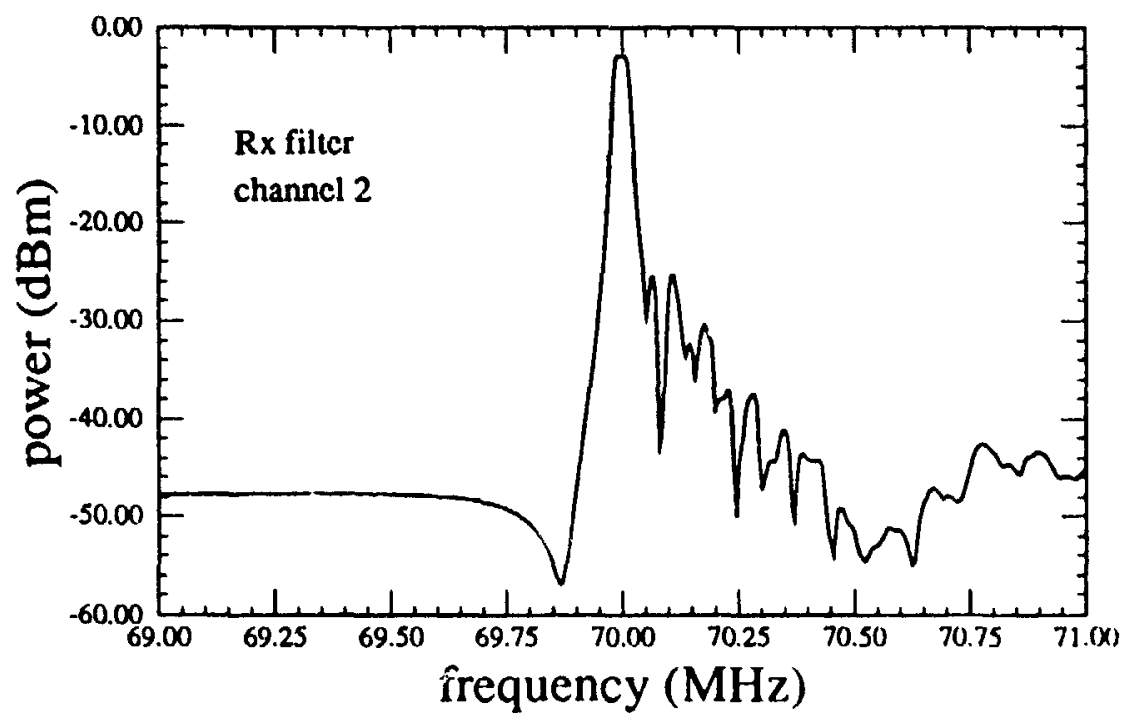

Figure- 3.12: Transfer function of receive crystal filter channel 2 


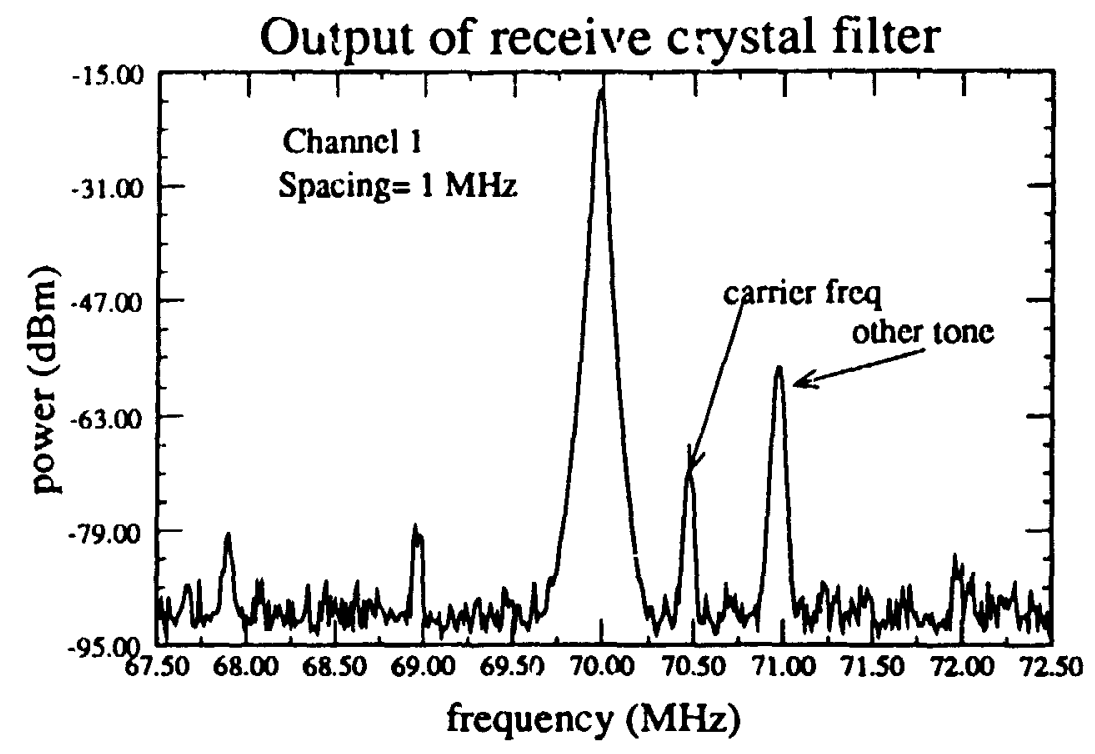

Figure- 3.13: Ouput of crystal filler, frequency diversity receiver,channel 1

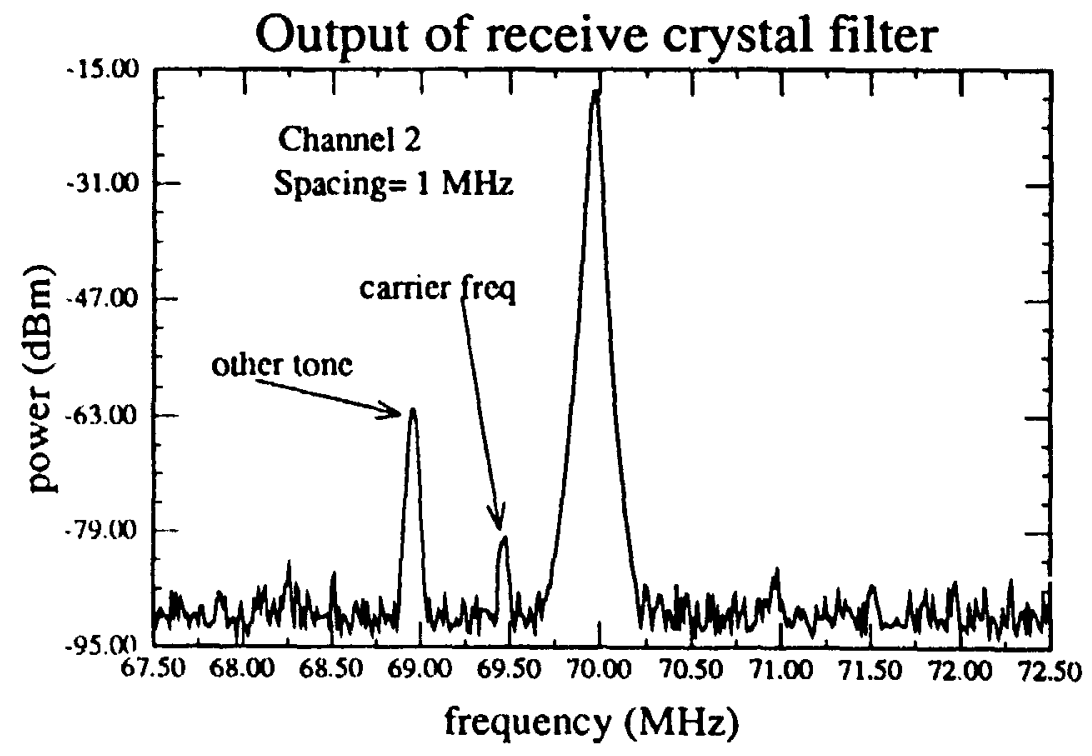

Figure- 3.14: Output of crystal filter, frequency diversity receiver, channel 2 


\subsection{Space and polarization diversity receiver}

The receiver configuration was the same for space and polarization diversity with only the antenna positions differing. The IF and the demodulation sections of this receiver are the same as the frequency diversity receiver but the front end part is different. Figure 3.15 and plate 3 show a block diagram and a photograph of the receiver.

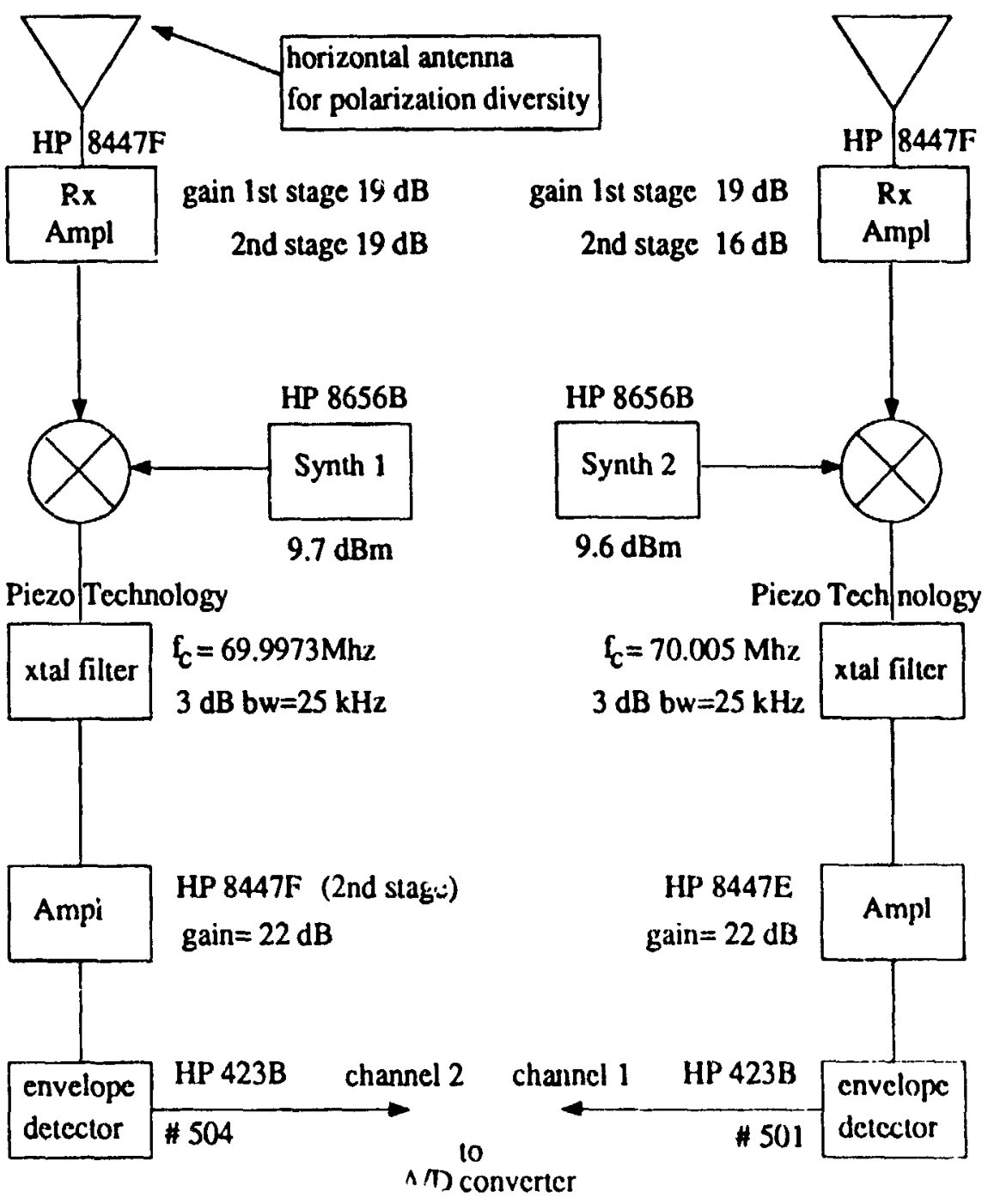

Figure- 3.15: Block diagram of space and polarization diversity recelver 
The front end portion differs from the frequency diversity case in that there are two receiving antennas. Each antenna requires a preamplifier and amplifier before going to the IF section. Although nominally the same, it was discovered that the gain of the two amplifiers were different. They were fine tuned by using some coaxial attenuators at the output of the first and second stage of each amplifiers. This was important in order to situate the output of each branch within the range of the analog-to-digital converter and to insure that both branches were at the same level.

The center frequencies of the two filters were about $3 \mathrm{kHz}$ apart. Contrary to the frequency diversity case, the input frequency of each mixer was the same. Only one synthesizer tuned to the half-way point between the center frequencies of each filter and a power divider could have been used to drive both mixers. This would have given a mixer output frequency slightly off from the center frequencies of their respective filters. The use of two synthesizers allowed for more precision.

\subsection{Antennas}

The three antennas used for the experiments were $\lambda / 4$ monopole with ground plane. Two of them were borrowed from the Department of Communication 's Communication Research Council (CRC) laboratory in Ottawa. The third one was made locally using the CRC's design. Plate 4 shows a photograph of the antenna. They are made of sheet copper and 1/8" copper tubing. The radiating (vertical) element is soldered to the center pin of a fernale BNC flange connector. It is electrically isolated from the radial (angled) elements. The four angled radial arms are electrically equivalent to a ground plane. They are soldered to the star shaped base which is grounded to the flange connector. Instead of being at a $90^{\circ}$ angle with the radiating element, to be closer to an actual plane, they are angled such as to provide a better horizontal radiation pattern. The styrofoam and cardboard bases provide support and facilitate handling. These bases are fastened to the antennas with a silicone compound.

For consistency, always the same antenna was used at the transmitter. The other Iwo were used with the receiver (only one in the case of frequency diversity). The 
space and polarization diversity measurements involved the placing of the two receiver antennas in a specific position in reference to each other. This was accomplished by building two frames holding the antennas in the desired position. The frames could be moved without changing the relative position of the antennas. The frame for space diversity consisted of a piece of wood with a holding handle. Some holes were drilled in the wood at the proper spacing to allow plastic tie-down to hold the antenna bases in place. Plate 5 shows a photograph of the frame with two antennas. The frame for polarization diversity was made using the comer of a cardboard box. This frame was then fastened with plastic tie-down to the previous wooden frame for easier handling. Plate 6 shows a photograph of the cardboard frame.

The radiating element's physical length was calculated based on a frequency of $915 \mathrm{MHz}$. The electrical length was not adjusted for that frequency. To improve the antenna's performance, the length of the radiating element was adjusted by trial and error with a network analyzer to give a low return loss between 903 and $928 \mathrm{MHz}$. The return loss is an indication of how much energy is reflected back to the transmitter due to an impedance mismatch between the transmission line and the load (in this case the antenna). Return loss is given as a ratio of the power reflected over the power supplied to the antenna. This was dcne by looking at the network analyzer, cutting a little piece of the tubing, looking again at the analyzer, etc. When the return loss notch was close to $915 \mathrm{MHz}$, the return loss at each end of the band was between -15 to $-20 \mathrm{~dB}$.

\subsection{Analog-to-digital converter}

\subsubsection{General}

The output provided by the env. 'ope detectors had to be recorded and stored for later retrieval and processing. This was the role of the data acquisition system. The output was first digitized with an analog-to-digital converter and, after some transformations, stored in the computer. The DASH-16F, from Metrabyte Corporation is a multifunction high speed analog/digital I/O expansion board designed for the IBM PC and compatible machines. Its analog-to-digital converter was used to digitize the envelope detectors 
output. It was installed in an expansion slot in the HP Vectra PC, which was the computer used for data storage and processing. The DASH-16F has many features and options that can be easily tailored to a specific application. We will limit ourselves to cover only the modes used in our experiment. More details on this board are available in Appendix C.

The board's installation into the PC consisted of a few steps. After selecting a suitable $\mathrm{I} / \mathrm{O}$ base address (hex 300 in our case), the input channel configuration switch was set to 16 single ended channels, the input voltage switch set to unipolar, $1 \mathrm{~V}$. The DMA priority level was set to 1 . The interrupt priority level was set at 2 . These were proposed as a standard setting.

Utility software was provided with the board and included:

- an assembier language driver subroutine to control the board functions;

- program example of each of the driver modes in BASIC;

- a set-up, a calibration and a test program; and

- various demonstration programs.

\subsubsection{Modes of operatiohi of the analog.to-digital converter}

The control program uses the driver subroutine with up to 7 parameters: mode, data (array of 5 values) and error flag. The mode parameter indicates which function the subroutine is to perform. The error flag provides some information about the status of the operation just performed. There are 21 possible modes, 0 to 20 , that can be used to operate the DASH-16F. We used 6 of these modes. A complete list of modes and their functions is given in Appendix C. Table 3.2 lists, in order of appearance in the data acquisition control program ADSAMP.BAS (listi..g of this program is in Appendix A), the modes used in the experiment. 


\begin{tabular}{|c|c|}
\hline \multicolumn{2}{|c|}{ DASH-16F MODES } \\
\hline Mode & Function \\
\hline Mode 0 & initialize board \\
\hline Mode 1 & set multiplexer scan limits \\
\hline Mode 17 & $\begin{array}{c}\text { set programmable timier } \\
\text { rate }\end{array}$ \\
\hline Mode 20 & data acquisition \\
\hline Mode 8 & $\begin{array}{c}\text { monitor status of data } \\
\text { acquisition }\end{array}$ \\
\hline Mode 9 & data transfer from memory \\
\hline
\end{tabular}

Table 3.2 List o: DASII-I6F modes used in the experiment

The modes will now be explained in more detail and the parameters required will be listed. The parameters are MD\%, DIO\%(0:4) and FLG\%. \% indicates an integer variable ( 2 bytes).

\section{Mode 0}

It initializes the board. The entry parameters are:

$$
\begin{aligned}
& \text { MD\%=0, mode number; } \\
& \text { DIO\%(0)= I/O address; } \\
& \text { DIO\%(1)= interrupt level; } \\
& \text { DIO\%(2)=DMA level; } \\
& \text { DIO\%(3) and DIO\%(4) : not used. }
\end{aligned}
$$

The return parameter is FLAG\%, providing a value indicating if the operation was successful or if something has gone wrong. The entry parameters do not change.

\section{Mode 1}

This mode sets the scan limits of the input channels' multiplexer to the desired values. There is one analog-to-digital converter module and a maximum of 16 input channels. The multiplexer is used to activate in sequence the input channels on which the next 
conversion will be performed. Input channel 0 and 1 were used in our experiment. The entry parameters are:

$$
\begin{aligned}
& \text { MD\%=1, mode number; } \\
& \text { DIO\%(0)= channel } 0 \text {, lower scan limit; } \\
& \text { DIO\%(1)= channel }: \text {, upper scan limit; } \\
& \text { DIO\%(2), DIO\%(3) and DIO\%(4): not used. }
\end{aligned}
$$

On return FLAG\% provides a diagnostic of the operation and the entry parameters are not changed.

\section{Mode 17}

This mode sets the rate of the internal programmable timer. If the option is selected, the timer will trigger the start of a conversion at the selected interval. The rate is selected by specifying two numbers N1 and N2 that will divide the timer's input clock frequency.

$$
\text { Rate }=\frac{\text { clock freq }}{N 1 \times N 2}
$$

The clock is a precision crystal oscillator operating at $1 \mathrm{MHz}$. N1 and N2 can be between 2 and 65535 and must be integer. This allows rates from less than 1 pulse/hour to the maximum possible by the analog-to-digital converter, about 100,000 samples/s. On entry the parameters are:

$\mathrm{MD} \%=17$, mode number,

$\mathrm{DIO} \%(0)=\mathrm{N} 2$;

$\mathrm{DIO} \%(1)=\mathrm{N} 1$;

DIO\%(2),DIO\%(3) and DIO\%(4) : not used.

On return the parameters do not change.

\section{Mode 20}

There vere many modes offered for the actual data acquisition. Different modes allowed much flexibility. We selected mode 20 because it fitted best our needs. An explanation on how it was selected can be found in Appendix C. Mode 20 performs $\mathrm{C}$ conversions (= 1 scan) per irigger, up to $\mathrm{N}$ total conversions, on successive channels. The value of 
$\mathrm{C}$ depends on the channels specified earlier by mode 1 . The trigger is provided by the timer or externally. Data is transferred to memory by interrupt. The number of scan is N/C. The time between a conversion on channel 1 and on channel 2 was is $60 \mu \mathrm{s}$. The calculations to obtain this value are explained in Appendix C. The entry parameters are:

$M D \%=20$, mode number,

DIO\% $(0)=$ total no of conversions, $N, 1$ to 32767 ;

DIO\%(1)= address of memory segment to receive data;

DIO\%(2)= trigger: $1=$ external or $0=$ internal timer;

$\operatorname{DIO} \%(3)=1$; recycle: data continuously written to the same memory segment. $=0$; no recycle: conversions stop when $\mathrm{N}$ is reached;

DIO\%(4)=not used.

On retum the parameters are not changed and FLAG returns any error codes.

\section{Mode 8}

This mode is used to monitor the status of conversions initialized by mode 20 . On entry the parameters are:

$\mathrm{MD} \%=8$, mode number;

The other parameters are not used. On return the 'uarameters are:

DIO\% $(0)=$ operation type, i.e. mode $5,6,18$ or 20 ;

DIO\% $(1)=$ status of operation: active $=1$, done $=0$;

DIO\%(2)= current value count;

DIO\%(3) and DIO\%(4) are not used.

\section{Mode 9}

This mode is used to transfer data from memory to an array defined in the control program. It transfers the specified number of values from the specified memory segment into a dimensioned array. It is also possible to retrieve data at a given offset from the starting memory address. Earn value is an integer i.e. 2 bytes. The analog-to-digital converter produces a 12 bit number. The other 4 bits represent the number of the 
originating channel. There is an option to have this number also pu+ into an array. The entry parameters are:

$M D \%=9$, mode number;

DIO\% $(0)=$ number of values to transfer, 1 to 32767 ;

DIO\% $(1)=$ starting address of memory segment;

DIO\%(2) = offset from beginning of segment, 0 to 32766;

DIO\%(3) = VARPTR(data array), VARPTR is a function pointing to the beginning of the data array;

DIO\%(4)=VARPTR(channel number array).

On return, FLAG\% gives the error code and the other parameters are not changed.

\subsection{HP Vectra PC}

\subsubsection{General}

The Vectra PC is an IBM AT compatible machine running at a clock frequency of $8 \mathrm{MHz}$ with 640 Kbytes of RAM, a 20 Mbytes hard drive and a 1.2 Mbytes floppy drive. It is also equipped with the Language Processor card, a completely separate processor emulating the HP 200/300 BASIC workstation. The processor, a MC68000, has a separate 3 Mbytes of RAM and run HP BASIC 5.0. It accesses the same disk drives and other peripherals. The card has a built-in HP Interface Bus (HP-IB) connector. This is the HP version of the IEEE-488 bus. Having used the Language Processor in the preliminary measurements and having gained experience with it, it was decided to use it for data storage and processing. Unfortunately there were not many communication links between the Language Processor (referred from now on as the HP BASIC environment) and the AT compatible piocessor (the DOS environment). Once in the HP BASIC environment, there was no direct access possible $t:$ any expansion boards in the DOS environment. This precluded using HP BASIC to control the analog-to-digital converter board. GW-BASIC was used to do this function. This version of BASIC u'as not 
nearly as powerful as HP BASIC. Section 3.9.2 will explain the data acquisition control program.

\subsubsection{Data acquisition and conversion programs}

The data acquisition control program was run in GW-BASIC. Using the machine language driver subroutine the measurements were taken and the data stored on disk in ASCII files. This format was selected because it was the simplesi way to store the data files. These files were not very practical in term of disk storage and access time but they were the easiest type of files to import into HP BASIC. Some utility programs were provided with the Vectra and one of them converted ASCII files produced in the DOS environment to HP BASIC ASCII files. The data acquisition program is called ADSAMP.BAS and is listed in appendix A. Before starting the measurements some decisions had to be made on sampling rate and the length of the sampling interval. The product of these two values was the number of samplings, referred earlier as $\mathrm{N}$ in the description of mode 20 .

In the review of previous experiments done in chapter 2, sampling rates for narrowband system experiments varied from about 10 samples/s [17], [4] to 16,000 sample s/s [24]. In our case the measurements would be taken during the day when people are moving in the building. A more significant factor would be the fact that the receiver antenna would be moving to get some spatial averaging. The actual measurement procedure will be discussed in section 3.11. This dictated a rate that should be faster than 10 samples/s but how fast did it need to be? A test run was made at 2000 samples/s for 5 to 10 seconds. The output of the envelope detector was plotted against time, using all the samples. The plot was then redone using every second point, every fourth point and every eight point. This was equivalent to using a rate of 1000,500 and 250 samples/s. There were no noticeable difference using 1000 samples/s. The 500 samples/s plot was very close to the original one. The last plot showed a large difference with the first one. A 500 samples/s rate was fast enough to satisfy our requircment. 
The duration of the sampling interval had to be long enough to reflect the status of the channel at that time. An interval too short might reflect only a transition of the channel and would diminish the statistical value of the measurements. 10000 readings would give a minimum probability of $10^{4}$. Twice that amount would give a minimum probability of $5 \times 10^{-5}$. The file size was also a factor to keep in mind so that file management and storage would not be too complex. In double precision arithmetic, $2 \times 30000$ values was about the maximum practical number of values that could be processed at the same time.

With a total number of samples of 60000 (30000 per channel), a sampling rate of 1000 samples/s would give a 30 seconds sampling interval. At 500 samples $/ \mathrm{s}$, the interval would be 60 seconds. It was estimated that there would be enough fading events in 1 minute to make the measurements worthwhile.

Before explaining the data acquisition program, we will deal with some of the limitations of the driver subroutine and GW-BASIC. The maximum number of conversions (referred earlier as $\mathrm{N}$ ) allowed by the driver subroutine in one run was 32 767. The 60000 conversions were taken in 2 blocks of 30000 samples by using a loop repeated twice. There was a gap of 10 to $20 \mathrm{~ms}$ between the each iteration of the lnop.

GW-BASIC has a total work space of $64 \mathrm{~Kb}$. There had to be a trade-off between program space and space allocated for arrays. A 25000 integer array was about the maximum array size that could be included in the workspace with the data acquisition program. The 60000 readings were therefore handled in blocks of 15000 values.

The data acquisition program ADSAMP.BAS using the driver subroutine took 60 000 readings in 60 seconds and wrote the data on disk. The listing in included in appendix A. The program structure is listed in the following statements.

1. the machine language driver is loaded in memory at address hex 8000 ;

2. program parameters are initialized;

3. board is initialized using mode 0 ;

4. channel scan limits are set using mode 1;

5. programmable timer rate is set using mode 17; 
6. start first 30000 conversions using mode 20 . data to be written in memory starting at address hex 3000 ;

7. start second 30000 conversions using mode 20 . data to be written in memory starting at address hex 4000;

8. transfer first block of 15000 values from memory to array in program using mode 9. write data from array to disk;

9. repeat with second block;

10. repeat with third block;

11. repeat with fourth block.

\subsection{Calibration}

The raw data produced by the analog-to-digital converter did not have much significance by itself. It needed some sort of scaling to represent something meaningful. It was decided to relate the values produced by the analog-to-digital converter to the power received at the antenna. The power input at the receiver's front end amplifier is a known parameter in many systems and it means something tangible: how much energy was picked up by the antenna.

To do this calibration effectively the input power into the receiver's amplifier had to be measured and controlled. It was not practical to try using the transmitter as the source due to the variations that would be introduced, even by transmitting over a shon distance. A signal synthesizer directly connected to the receive amplifier was used instead. Its output power was eas..y controlled by changing the front panel settings. For each power level of the synthesizer, 200 readings from the analog-to-digital converter were averaged. After each set of reading, the synthesizer power level was incremented by $1 \mathrm{dBm}$. That process was repeated on both channels and for each frequency. This produced six sets of measurements, five for frequency diversity and one for space and polarization diversity. These were the backbone of the scaling files. Figure 3.16 shows an example of these values for frequency diversity and figure 3.17 shows the values for space and polarization diversity (combined because they use the same transmitter). 


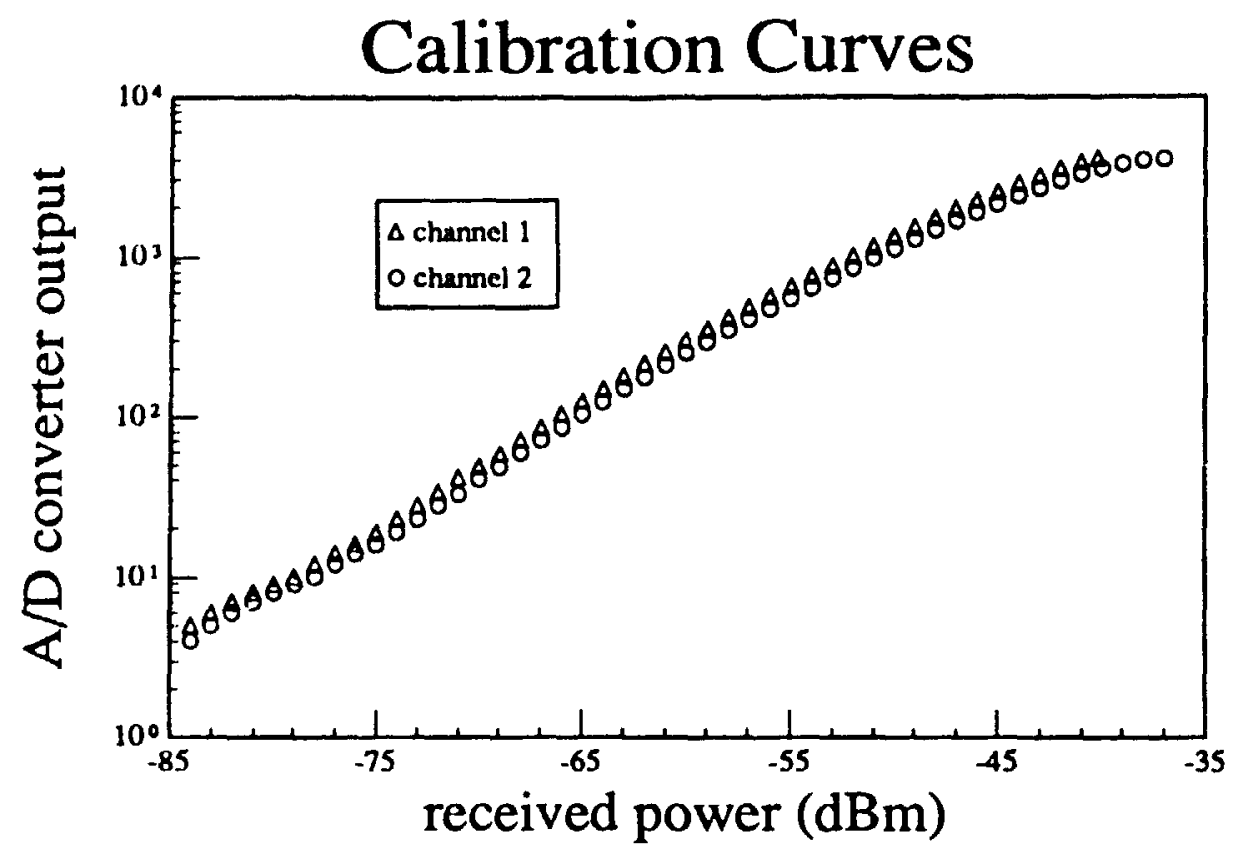

Figure- 3.16: Calibration curve, $1 \mathrm{MHz}$ spacing frequency diversity

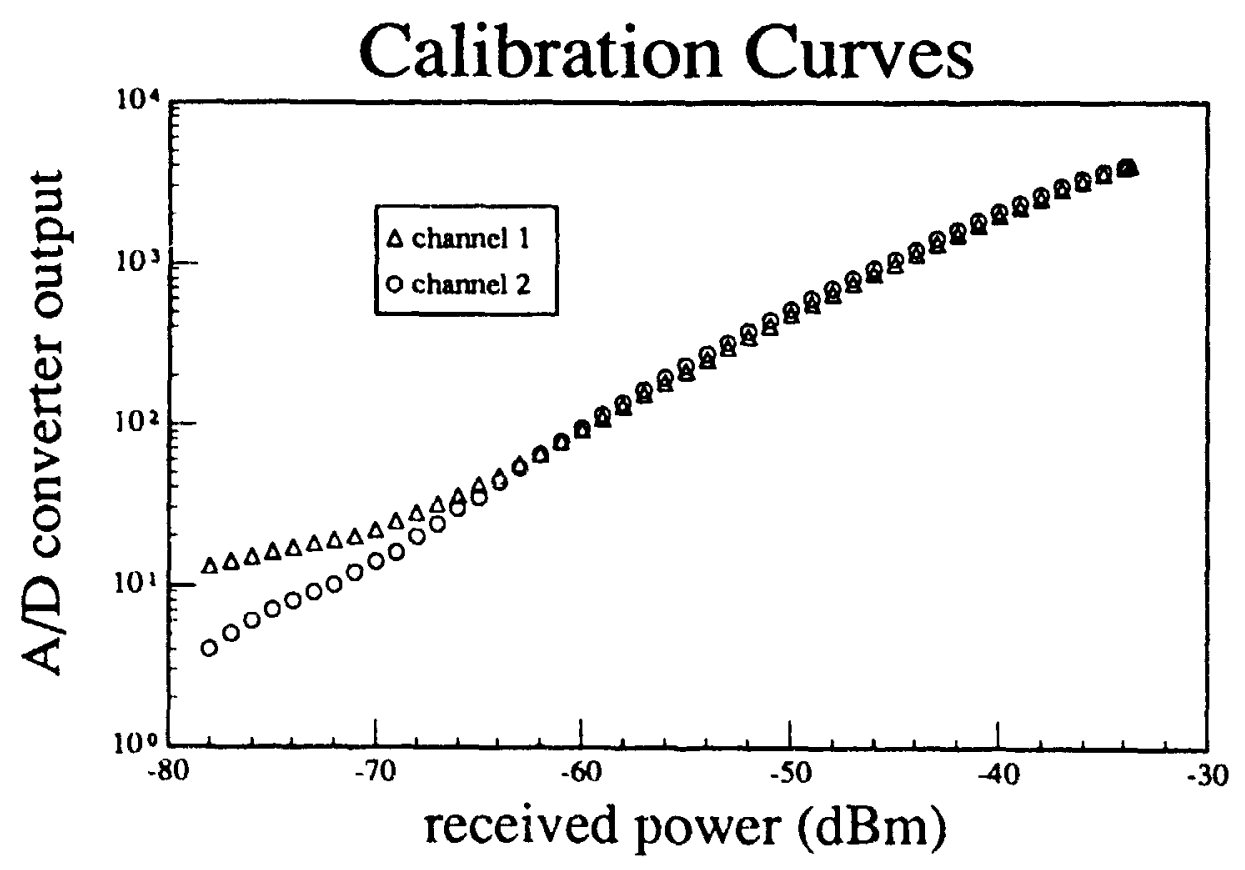

Figure- 3.17: Calibration curve, space and polarization diversity 
The analog-to-digital converter readings went from 0 to 4095 . The measurements explained earlier covered this range with power levels from about $-83 \mathrm{dBm}$ to -33 $\mathrm{dBm}$. The aim of the calibration was to generate a $\mathrm{dBm}$ value for each analog-todigita! converter reading. Taking the values already measured as reference, we used linear interpolation to generate a dBm value for all remaining analog-to-digital converter readings. That was done for all six sets of measurements and the results were stored in six files. Each file consisted of $4096 \mathrm{dBm}$ values for channel 1 and 4096 more for channel 2. Once this was done for $\mathrm{dBm}$ values, they were converted in milliwatt and stored these results in six other files.

During data processing, the first operation performed was to read the proper scaling file and to replace each raw analog-to-digital converter reading by the corresponding power ( $\mathrm{dBm}$ or $\mathrm{mW}$ ) value. This could be done quickly and allowed the data files to be stored in the raw form, as integer numbers using two bytes per value instead of in the scaled form as real numbers using 8 bytes per value.

\subsection{Measurements procedure}

\subsubsection{General}

Before taking measurements, 20 locations were selected on the same floor around the laboratory. We were on the top floor of the School of Architecture building at Carleton University, Ottawa, Ontario. The other rooms on this floor consisted of two drafting rooms, one other lab, a computer terminals room, five study rooms for students, a number of offices and two mechanical rooms. Since this floor was an addition on top of an existing building, the two mechanical rooms contained ventilation equipment that was originally on the roof. The drafting rooms were furnished with about 40 wooden drafting tables. The two laboratories had wooden cabinets and tabies but only a small quantity of equipment. There were six computer terminals in the terminal room. The offices and the study rooms had wood and metal furniture. The hallways were laid out in a $\mathrm{H}$ form. In the ccntei of that $\mathrm{H}$ was a large skylight going right through to the floors below. The windows in the lab, the skylight, in some study rooms and on some outside windows 
had wire mesh in their center. The walls were mainly made of plasterboard with wood studs and some metal pillars. The floors were made of cement covered with tiles. The ceilings were all cement with soundproofing panel held by a metal frame about .5 met:from the, ceiling. In this inter-space, many cables were routed. Appendix E shows a floor plan of the area and a table of locations and distances The conditions (rooms full or empty, hall busy...) at the time of measurements are also listed in appendix E. The receiver was located in one of the labs. The transmitter was moved to various locations on the floor. At each location, the exact antenna location was recorded by measuring the distance from the antenna to an easily recognizable building feature. With these measurements, the transmitter could be brought again to the exact same location for the other diversity measurements. This location was reported on a scaled floor plan and the exact distance from the transmitter to the receiver was calculated.

\subsubsection{Procedures}

After bringing the transmitter to th: desired location, the anrenna position was recorded. This was usually done by aligaing it with the floor tiles (12 " by 12 ") and counting the number of tiles to the next wall corner or angle. After a warm-up period the transmitter attenuation would be set to a given level. A short test run was done to confirm the attenuator setting. If the signal level was not fully within the receiver's dynamic range, the attenuator would be adjusted and another test run would be done. The conditions around the transmitter were recorded (full, half full or empty room etc). Using the frame shown in plate 7 as a guide, the receive antenna was moved by hand along the circumference of a circle cut inside the frame. The circle had a diameter of 1 $\lambda$ for the lowest used frequency (in our case $905 \mathrm{MHz}, \lambda=33 \mathrm{~cm}$ ). The antenna was moved along that circle for two reasons. First it was to get a average received power that would be a local average and second, to create fading events. This occurred for 1 minute then the program wrote the data on disk wiile the antenna spacing or the frequency settings were adjusted. The five sets of measurements for a given type of diversity (one set in the case of polarization diversity) were done within 30 minutes of each other at one location without moving the transmitter. 
The frequency diversity measurements were made first. The transmitter and receiver were then modified to conduct the space diversity measurements. The same sequence was repeated at all the locations. The antennas at the receiver were then adjusted for $90^{\circ}$ polarization separation and again the same sequence of events was repeated. 


\section{Chapter 4 Analysis and results}

\subsection{Introduction}

The analysis of the data collected was done keeping in mind the purpose of the experiments. We wanted to establish the effect of increased spacing on diversity improvement in indoor radio communication. Data was recorded for 20 transmitter locations for each ts pe of diversity. For frequency and space diversity, 5 frequency and antenna spacings were tested. There was only one configuration for polarization diversity: $90^{\circ}$ separation. Each data file was processed to produce a cumulative distribution of the envelope level for channel 1 and 2 separately and for a set of points which were the result of selection combining done on both channels. Average path loss was calculated for each location using the average received power values, the transmitter power output and the attenuator setting at the transmitter. With this information, scatter diagrams were produced and the distance-power law for each set of measurements (total of 11) was calculated.

The next section will explain the steps required to produce the distributions and the atte idation scatter diagrams. Section 4.3 will present tables of improvements obtained with diversity. It will also show samples of the received signal envelope and the various cumulative distributions. Section 4.4 will show the attenuation scatter diagrams and present the table of distance-power laws. Section 4.5 will summarize the results obtained in the previous sections. 


\subsection{Analysis}

\subsubsection{General}

The initial data recorded was ASCII files produced by ine sampling progiam ADSAMP.BAS (listing in Appendix A) in GW-BASIC. The files contained some miscellaneous information and 30000 pairs of readings from the analog-to-digital converter. Since they were read in sequence channel 1 then channel 2 , they were stored in the same way. Before processing the files in the HP BASIC environment, a 2 step conversion was performed. The files were initially stored as ASCII files to make storage simpler and to facilitate their conversion to HP BASIC format. A utility program provided with HP BASIC was used to convert the files to HP BASIC ASCII format. This was the first step of the conversion. The HP BASIC ASCII files were also very cumbersome: not only did they use 1 byte per digit (up to 4 digits per value) but each value had a 4 byte header. This was a waste of disk space and very slow to access. The second step of the conversion was to change the files from ASCII to binary format. A program read the HP BASIC ASCII file, re-ordered the data to bring each channel's data in sequence (instead of having them alternating) and re-wrote the data on fluppy disk as HP BASIC binary files. Each value was now stored as an integer at 2 bytes per value. Appendix B describes the structure of the binary data files.

\subsubsection{Processing for cumulative distribution}

The first calculation task was to obtain the average received power of each channel for all files. The steps to do that were to read in the raw data, read in the proper scaling file, scale the data and calculate the average. At this poirit, a results file was created for each data file and the average received power was stored in it. Appendix B describes the structure of the results files. In order to have an arithmetic ave:age and not a geometric average, the scaling files used were the ones in milliwatts, as discussed previously in section 3.10

The next step was the calculation of cumulative distribution for each channel individually and for a set of po. its obtained by doing selection combining with both 
channels. Calculating the distributions involved three operations which will be listed here and descrioed later.

1. establishing sub-range boundaries for the histogram;

2. producing the nistogram by finding in which sub-range each data point will be counted in; and

3. normalizing the histogram and summing its values to obtain the cumulative distribution.

Diversity will bring the greatest improvement when the two channels have the same average received power.

Histogram boundaries.

The histogram boundaries (or thresholds) were based on the receiver dynamic range. It was a litt:: above $40 \mathrm{~dB}$ so it was decided to partition this range into sub-ranges of 1 $\mathrm{dBm}$ each from $30 \mathrm{dBm}$ below average power to $10 \mathrm{dBm}$ above average power. These 41 thresholds were calculated for each channel based on its average power value. 41 thresholds gave us 40 sub-ranges of $1 \mathrm{dBin}$ plus one that included all events below the first threshold (average power - $30 \mathrm{dBm}$ ) and another one that included all events above the last threshold (average power $+10 \mathrm{dBm}$ ), for a total of 42 sub-ranges. The thresholds values were placed in two 41 value vectôs, X! and X2. X1 for example, was:

$$
\begin{aligned}
& X I(1)=\text { average power of channel } 1-30 \mathrm{dBm} \\
& X 1(41)=\text { average power of ciannel } 1+10 \mathrm{dBm}
\end{aligned}
$$

Associated with the 42 sub-ranges was a counting vector of 42 values. There was one vector per channel plus one for the selection combining. They were called Pdl, Pd2 and Pdjt. Pd1 for example, was:

$$
\begin{aligned}
& \operatorname{Pdl}(1)=\text { counter for first sub-range (all values } \leq X 1(1) \text { ) } \\
& \operatorname{Pdl}(41)=\text { counter for second last sub-range (all values }>X 1(40) \text { and } \leq X 1(41) \text { ) } \\
& \operatorname{Pdl}(42)=\text { counter for last sub-range (all values }>X 1(41) \text { ) }
\end{aligned}
$$

Prolucing the histogram. 
This step took the most time. Two similar subroutines were used to do the histugrams. One subroutine carried out the computations for channels 1 and 2 while the other was intended for the compuations related to the selection combining. The algorithm w'as designed to make maximum use of the built-in matrix and vector manipulation commands of HP BASIC. P1(1:30 000) and P2(1:30 000) contained the power values (scaled) of channel 1 and 2. The algorithm consisted of taking each value of $\mathrm{Pl}$ and searching . which sub-range it belonged by comparing it in sequence to each threshold value in $\mathrm{X} 1$. The sub-range was found at the first instance where the threshold value was greater than the P1 value. Once found, the cell (in the counting vector Pól) associated with that threshold value (i.e. $\mathrm{Pd1}(3)$ and $\mathrm{X1}(3)$ ) is increriented by one. If the value of P1 is not smaller than any threshold values, it belongs to the last sub-range. So as not to waste time during each search, the search was started from St1, the first threshold $\geq$ the minimum value of $\mathrm{Pl}$.

M1 - Minimum of P1

Search $\mathrm{X} 1$ for first value $\geq M 1 ;$ St 1 -index $\mathrm{XI}$

Search X1, starting at St 1 , for value $\geq P 1(I)$; D1 -index of X1

increment $\mathrm{Pd} 1(\mathrm{D} 1)$

if $\mathrm{Xl}$ (41) not $\geq \mathrm{Pl}(\mathrm{I})$ then increment $\mathrm{Pdl}(42)$

Siait over with next I

The same process was repeated for channel 2.

Selection of the best of both channels was done at each time point. The aim of the search was to find the threshold which was greater or equal to the value in both channels at this time point. The sub-range associated with that threshold had its cell incremented in the counting vector. The absolute power values were not used to do the selection. Instead the difference between a value and the average power for that channel was used. The selected channel was the one whose value for that time puint 'as the highest comparad ic its average value. This meant the indexes of vector X1, X2 (not the values in the vectors) were used as a selection factor. The minimum values of $\mathrm{Pl}$ and P2 and St1, St 2 were found earlier. The selected channel's value will always be 
greater than St, the largest of Stl and St2. The first part of the search is the same as for the individual channels. We started by searching $X 1$ from $S t$ to find the first value $\geq P 1$. The index D1 for that sub-range was used as the starting index of a loop comparing P2 with the value of $X 2$. When the threshold in $X 2$ became $\geq P 2$, the appropriate counter was incremented.This counter was Pdjt(), for the joint distribution.

search $\mathrm{Xl}$, starting at St, for value $\geq \mathrm{P} 1(\mathrm{I}) ; \mathrm{Dl} \leftarrow$ index of $\mathrm{Xl}$

for $J=D 1$ to 41

find if $P 2 \leq X 2(J)$

if yes, $\operatorname{Pdjt}(\mathrm{J})$ is incremented; start over with next I

if no, $\mathrm{J}$ incremented, comparison redone

if $X 2(41)$ not $\geq P 2(J)$ then $\operatorname{Pdjt}(42)$ is incremented

start over with next I

Summing the histogram

With the histogram values in Pd1(1:42), Pd2(1:42) and Pdjt(1:42), these numbers were normalized by dividing by 30000 , the total number of points. In these individual distributions, the minirnum probability that could be measured will 1/30 $000 \cong 3.33$ $\times 10^{-5}$. The cumulative distribution was done by adding the next $\mathrm{Pd}$ value to the previous cumulative sum.

$$
\begin{aligned}
& \operatorname{Cd} 1(1)=\operatorname{Pdl}(1) \\
& \operatorname{Cdl}(1) \leftarrow \operatorname{Cd} 1(I-1)+\operatorname{Pd} 1(1)
\end{aligned}
$$

The three $\mathrm{Cd}(.$.$) vectors were stored in the results files created earlier.$

Once these three steps were completed for all files, the distributions for each type of diversity were read into three $20 \times 5 \times 42$ matrices (location, spacing, number of points), for channel 1, channel 2 and the joint distribution. The matrix was $20 \times 1 \times 42$ for polarization diversity. For each type of diversity, a new file containing the three matrices was created. The distributions were now stored together by diversity type. Each spacing was then averaged over all locations: the 20 sets of 42 points were averaged together to give one distribution ( 42 points) representing all locations for that spacing. This 
operation was done on each matrix. In addition to the average, the maximum value and the minimum value of each point in the distribution were recorded. All these values were stored in a second set of matrix $5 \times 42 \times 3$ (spacing, number of points, average/max/min value). The minimum probability of the averaged distribution could be lower than $\mathbf{3 . 3 3}$ $\times 10^{-5}$ because each point is added up with the other 19 and then the sum is divided by 20 .

\subsubsection{Channel 1 correction}

After calculating the cumulative distribution function for all files and averaging them for all locations, it became evidenc that something was wrong with all the channel 1's distributions. They were supposed to be more or less the same as channel 2's distributions since they were taken at the same time and in the same conditions but they were diverging from the channel 2's distributions, especially at low power values. It was as if the analog-to-digital converter would never give a low power reading even if the signal was very weak. A DC offset present during the measurements would have this effect: the constant voltage would cause the analog-to-digital converter to always give a reading even when the signal level we being measured was very low.

In order to correct this problem, a numerical solution was tested and proved to be satisfactory in correcting the problem. A correction factor was subtracted from all channel 1's readings. This factor was such that it brought the lowest value in channel 1 in line with the lowest in channel 2. The histogram for channel 1 and for the selection combining points were re-calculated. The corrected distributions for channel 1 became much closer to channel 2's distributions. Figure 4.6 to 4.16 show all the distributions.

\subsubsection{Processing for attenuation results}

The individual results files mentioned earlier contained the average power of channel 1 , channel 2 and the corresponding transmitter attenuator value. As for the cumulative distributions, these values were collected in one matrix per diversity type. The matrices $(20 \times 5 \times 3)$ contained the above mentioned values for each location and spacing. The transmitter output power was then recorded for both transmitter configurations at different 
attenuator settings. For the space/polarization diversity configuration, there was only one tone at the transmitter's output: the measurements were done directly with a power meter. For the frequency diversity configuration, at least three tones were present at the transmitter output: to get an accurate figure of each tone's power the measurements wese taken with a spectrum analyzer. Under computer control, the spectrum analyzer took 80 power readings of each tone. The readings were averaged to produce the power output. These power values were stored in a vector using the attenuator value as the index.

With all the above values available, the average path attenuation was calculated for both channels for all 20 locations and 5 spacings. Using the attenuator value as an index, the average path loss was computed simply by doing the subtraction

Path loss $=$ Tx power (attenuator) - Average received power

To get the transmitter-receiver separation for all locations, a scaled fioor plan was used $\left(1 / 8^{\prime \prime}=1^{\prime}\right)$. During measurements, the transmitter location had been recorded precisely by measuring distances from the antenna to an obvious building feature. These distances were reported on the floor plan, the locations marked and the transmitterreceiver separation measured. To get the distance-power law, the first step was to plot relative attenuation against distance (on a $\log$ scale). A plot (or scatter diagram) was produced for each spacing (total of 11). Three of these scatter diagrams are shown in section 4.4. The absolute path attenuation calculated above was scaled to a relative attenuation using as the reference the lowest attenuation value (from the location closest to the receiver, $1.72 \mathrm{~m}$ ). The scatter diagrams obtained were fitted using a log fit. Channel 1 and channel 2 were fitted separately. The gradient of these fitted curves was the value representing the exponent of the distance-power law. The calculation to obtain the gradient were as follows:

$$
\text { gradicnt }=\frac{\Delta A t t(d B)}{\Delta D i s t(d B)}
$$

where $\triangle D i s t$ in $\mathrm{dB}$ is $10 \log ($ Dist2) $-10 \log ($ Dist1). Dist2 and Dist1 are two arbitrary distances used to calculate the $\mathrm{x}$ axis variation. $\Delta \mathrm{Alt}$ is the corresponding attenuation variation. 


\subsection{Diversity systems measurements}

\subsubsection{General}

To calculate the diversity improvement, a plot of the averaged distribution for channel 1 , channel 2 and the selection combining distribution was produced for each separation. Figure 4.6 to 4.16 show the plots for frequency diversity separations of $1 \mathrm{MHz}$ to $20 \mathrm{MHz}$, space diversity separations of $.75 \lambda$ in $2 \lambda$ and polarization diversity. Two other curves were plotted, for comparison purposes only. The first curve is a Rayleigh cumulative distribution (identified as 1 Rayleigh in figure 4.6 to 4.16). The second curve (identified as 2 independ. Rayleigh in figure 4.6 to 4.16) represents the distribution resulting from selection combining with 2 independent Rayleigh channels. The Rayleigh distribution was obtained by calculating the cumulative distribution function

$$
P(x<A)=1-e^{-10 \text { 娄 }}
$$

for $A$ values ranging from -31 to $+11 \mathrm{~dB}$. $A$ is the value of a given threshold relative to the average power. The 2 independ. Rayleigh curve was obtained by squaring the Rayleigh distribution. With two independent, uncorrelated channels the probability becomes $P(A, B)=P(A) \times P(B)$. By presenting these two curves, we are not implying that channel 1 and channel 2 should be Rayleigh or that the selection combining curve should be close to the other curve. We want to use them to show the maximum diversity improvement that could be obtained wher two channels had a correlation of exactly 0 .

The improvement calculations were done at the $.1 \%\left(10^{-3}\right), .5 \%\left(5 \times 10^{3}\right), 1 \%\left(10^{-2}\right)$ and $5 \%\left(5 \times 10^{-2}\right)$ probability level, that is to say $10^{-3}$ is the probability that the received signal is lower than a given value below average power. The improvement values were obtained graphically be measuring the relative improvement between the two sets of curves. This was done by reporting on the $x$ axis the position of each curve (the individual channels and of the joint channel) at the four probability levels. We used a feature of the graphic software allowing the tracking and reporting of the $x-y$ coordinates 
of a mouse controlled marker. The mid-point between channel 1 and 2 was used to compute the improvement value.

The following five figures, 4.1 to 4.5 , show a sample of the envelope detector's output for each type of diversity and for various spacings. We can deduce intuitively by looking at the fades in figure 4.1 and in figure 4.2 that the diversity advantage could be greater at $20 \mathrm{MHz}$ than at $1 \mathrm{MHz}$.

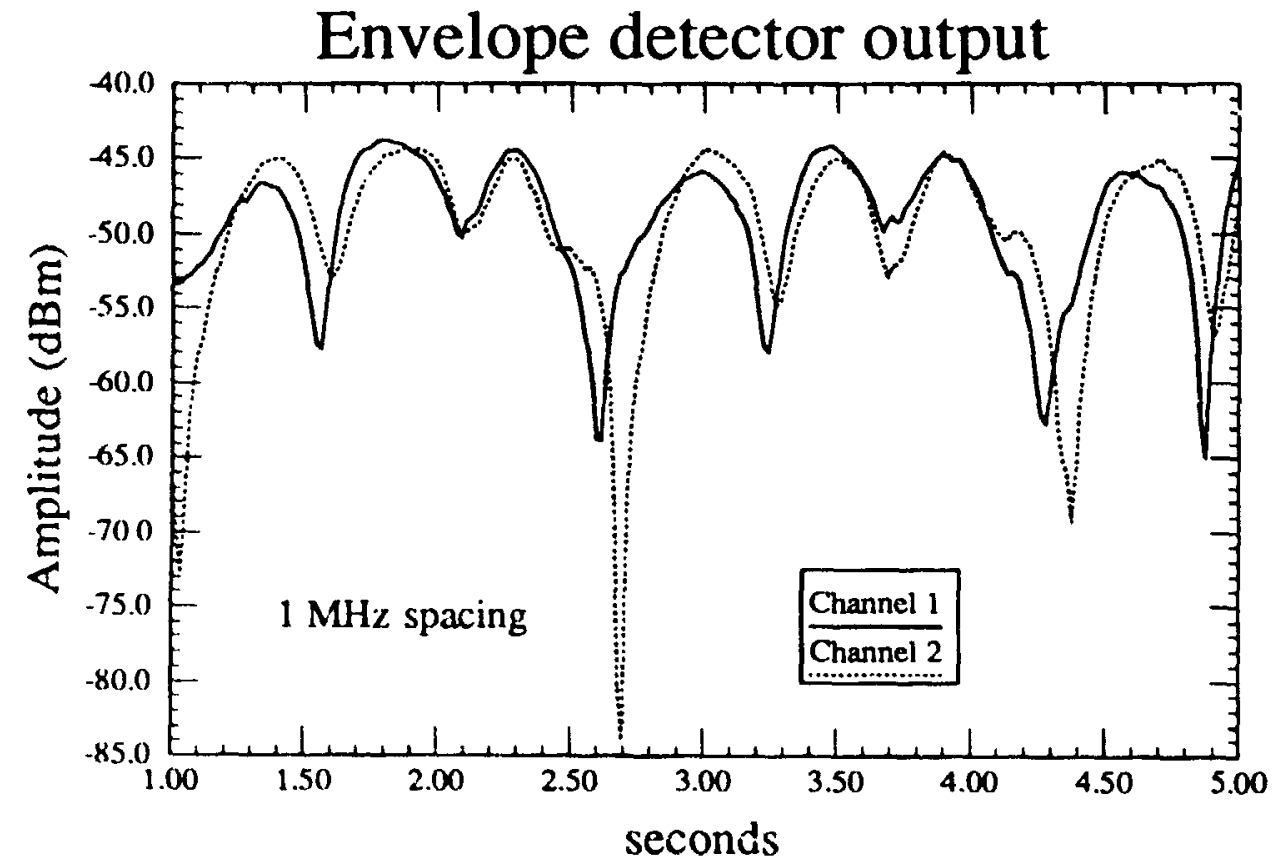

Figure- 4.1: Envelope detector ouput, frequency diversity, l MHz 
Envelope detector output

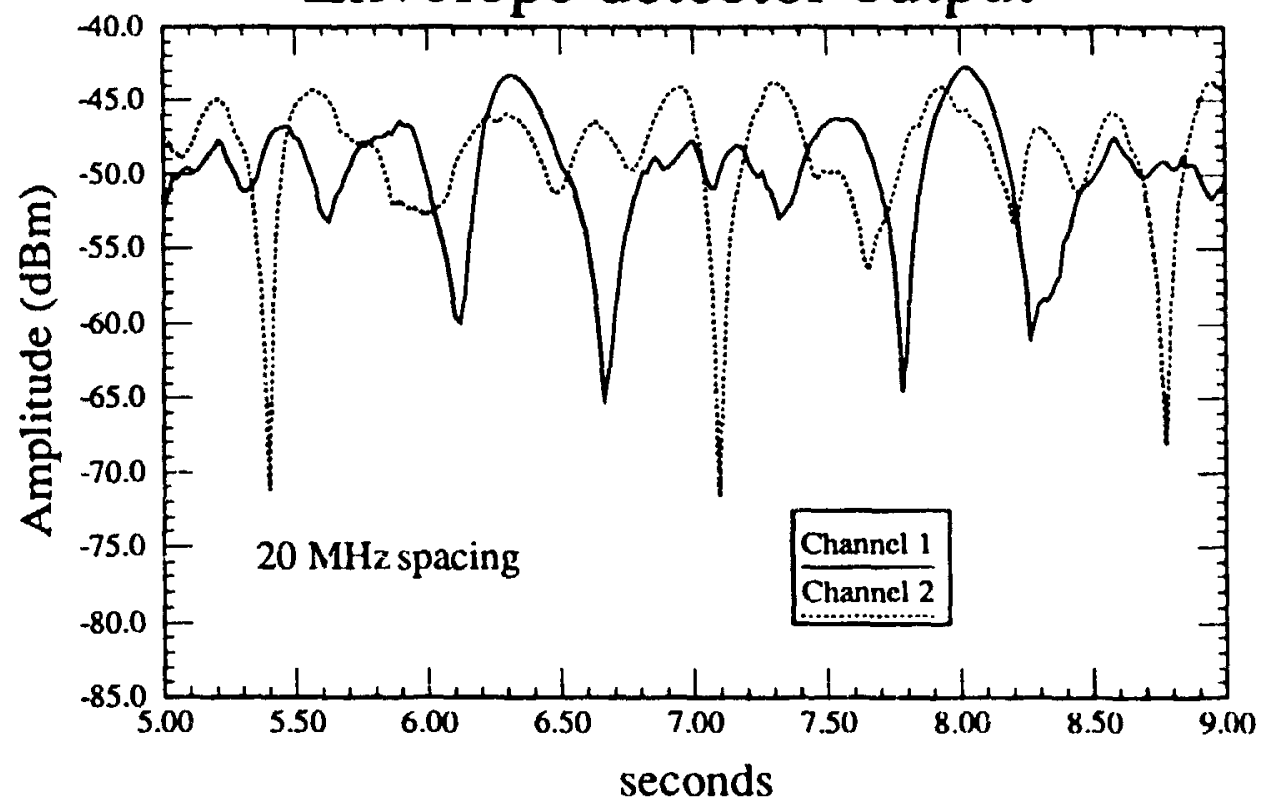

Figure- 4.2: Envelope detector output, frequency diversity, $20 \mathrm{MIIz}$ spacing

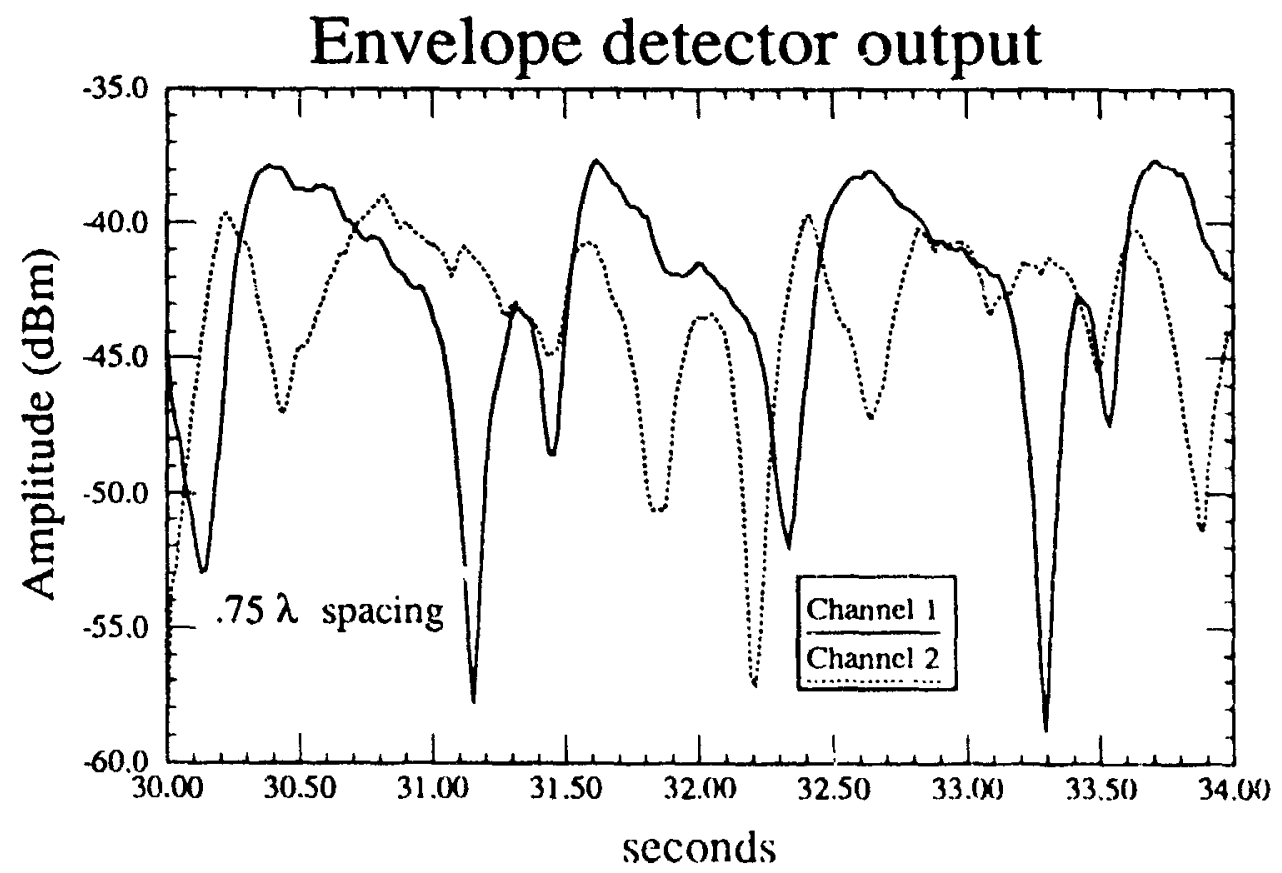

Figure- 4.3: Envelope detector output, space diverstly, $75 \lambda$ spat ing 
Envelope detector output

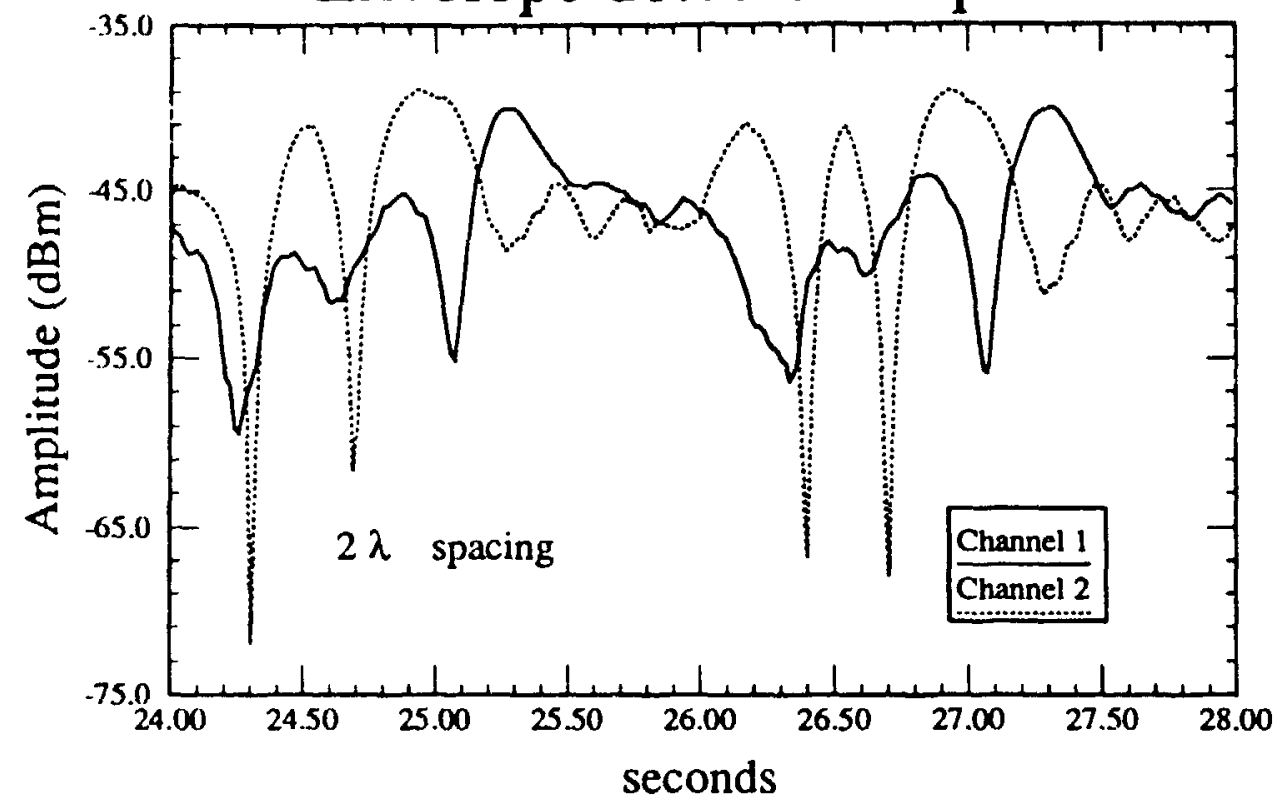

Figure- 4.4: Envelope detector oupue, space diversity, $2 \lambda$ spacing

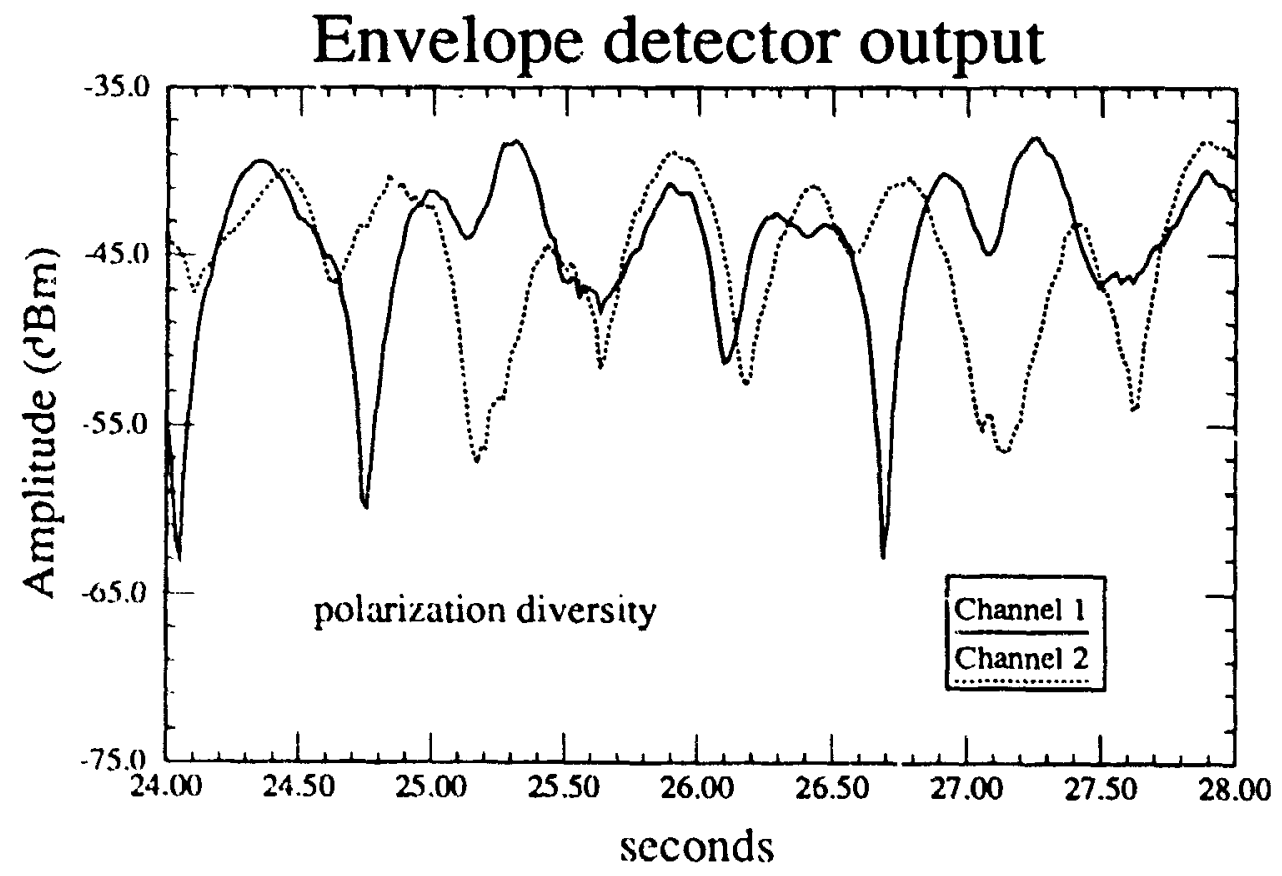

Figure- 4.5: Envrlope detector output, polarization diversity 


\subsubsection{Frequency diversity}

The graphs of average cumulative distribution functions for frequency diversity cases are shown in figures 4.6 to 4.10 . From table 4.1 we can see that the improvement is monotonically increasing with frequency separation. A three step progression can be seen in the improvement values: $1 \mathrm{MHz}, 5 \mathrm{MHz}$ and 10 to $20 \mathrm{MHz}$. This progression is well defined at the $5 \%, 1 \%$ and $.5 \%$ level. At the .1\% level, the progression is not as well defined: the 5 and $20 \mathrm{MHz}$ improvements are almost the same. The range of improvement values for the 10 to $20 \mathrm{MHz}$ spacings is very tight at the $5 \%, 1 \%$ and $.5 \%$ level: within $.7,1.1$ and $1.1 \mathrm{~dB}$ of each other and within $.6, .9$ and $1.7 \mathrm{~dB}$ of the Rayleigh value respectively. At the $.1 \%$ level this range is more than twice these numbers. The larger spread of improvement values at the $.1 \%$ level may be due to the fact that computations near the lower end of the curves are based on fewer samples and therefore do not present accurate statistics of the received signal.

\begin{tabular}{|c|c|c|c|c|}
\hline \multicolumn{5}{|c|}{ FREQUENCY IMPROVEMENT TABLE } \\
\hline & 5 \% Level & $\mathbf{1} \%$ Level & $\mathbf{5 \%}$ Level & $.1 \%$ Level \\
\hline $1 \mathrm{MHz}$ & 2.7 & 3.9 & 4.8 & 7.4 \\
\hline $5 \mathrm{MHz}$ & 5.5 & 7.4 & 8.7 & 11.8 \\
\hline $10 \mathrm{MHz}$ & 6.3 & 9.3 & 10.2 & 13.4 \\
\hline $15 \mathrm{MHz}$ & 7.0 & 10.4 & 11.2 & 13.6 \\
\hline $20 \mathrm{MHz}$ & 6.3 & 10.0 & 10.1 & 11.3 \\
\hline \hline Rayleigh & 6.9 & 10.2 & 11.8 & 15 \\
\hline & \multicolumn{5}{|c|}{ all values in $\mathbf{d B}$} \\
\hline
\end{tabular}

Table 4.1 Frequency diversity improvement values

The improvements calculated between the Rayleigh and the theoretical combining distribution were $6.9 \mathrm{~dB}$ at $5 \%, 10.2 \mathrm{~dB}$ at $1 \%, 11.8 \mathrm{~dB}$ at $.5 \%$ and $15 \mathrm{~dB}$ at $.1 \%$.

Reference [12] shows a channel availabïity cu. .e (this curve is complementary to ours: i.e. our $1 \%$ level= their $99 \%$ level) tor selection combining using a different $\mathrm{X}$ axis variable: the $(\mathrm{S}+\mathrm{N}) / \mathrm{N}$ ratio is used instead of absolute power. The diversity 
improvements obisined from their curve is about $10 \mathrm{~dB}$ at the $1 \%$ level and $14 \mathrm{~dB}$ at the $.1 \%$ level. These numbers are close to ours for the same levels.

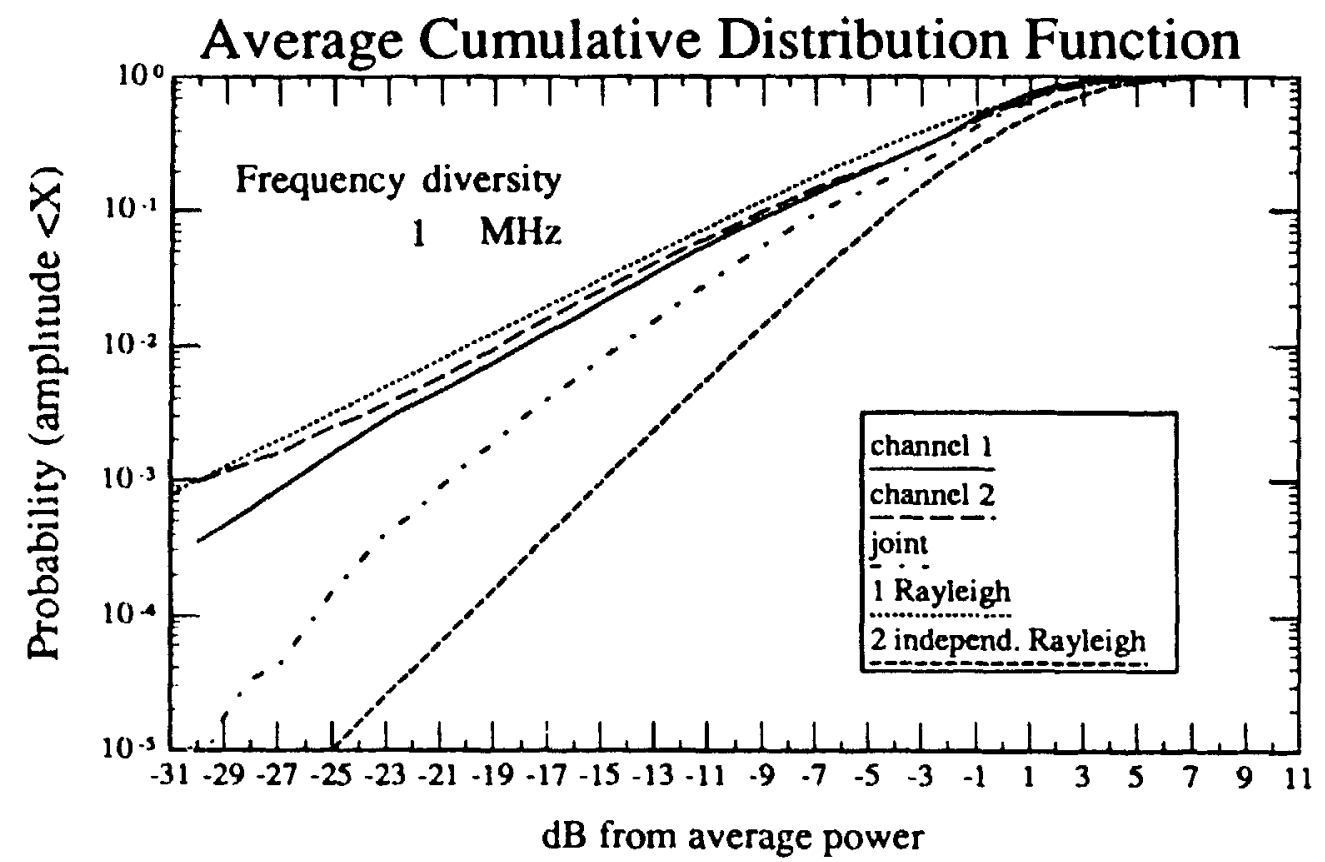

Figure- 4.6: Curnulative distribution function for I MHz spacing 


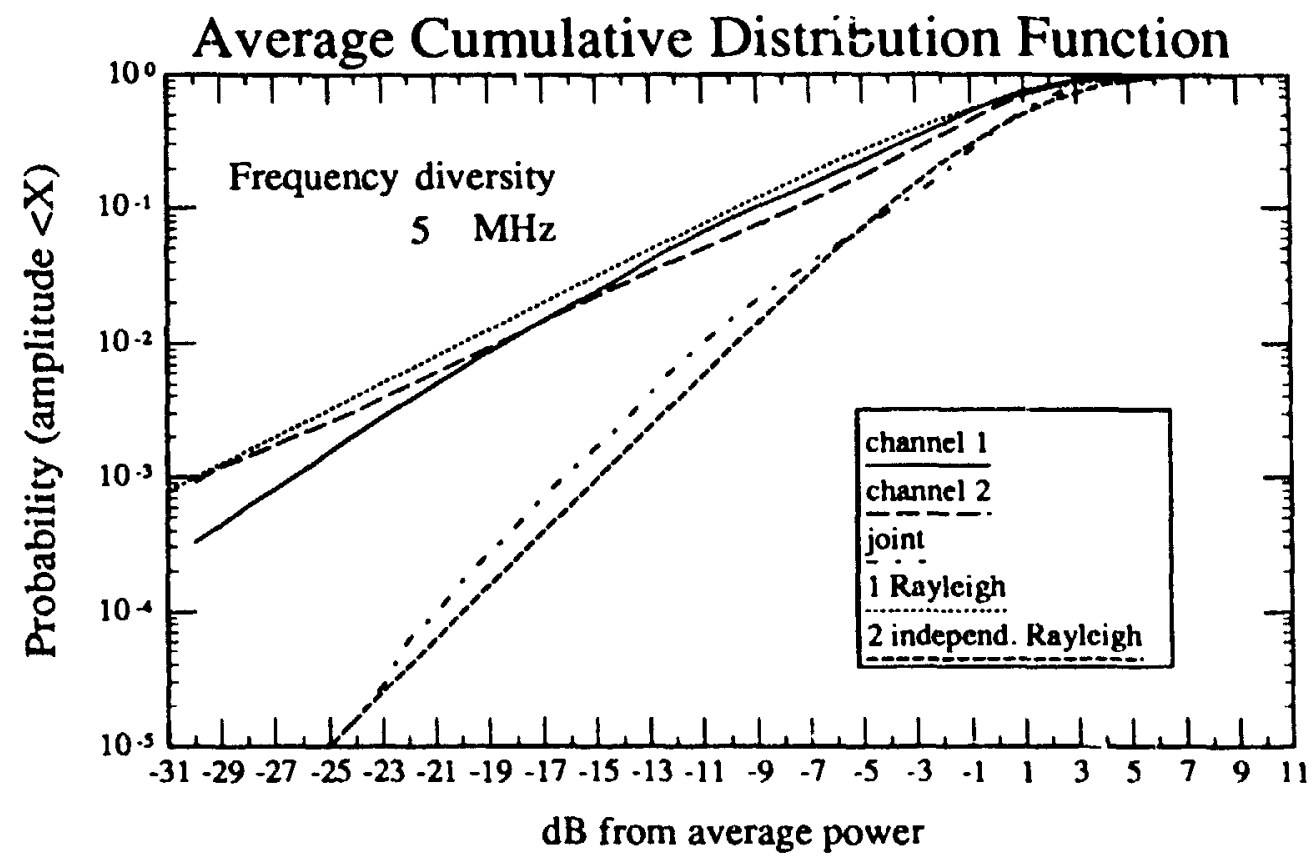

Figure- 4.7: Cumulative distribution function for $5 \mathrm{MHz}$ spacing

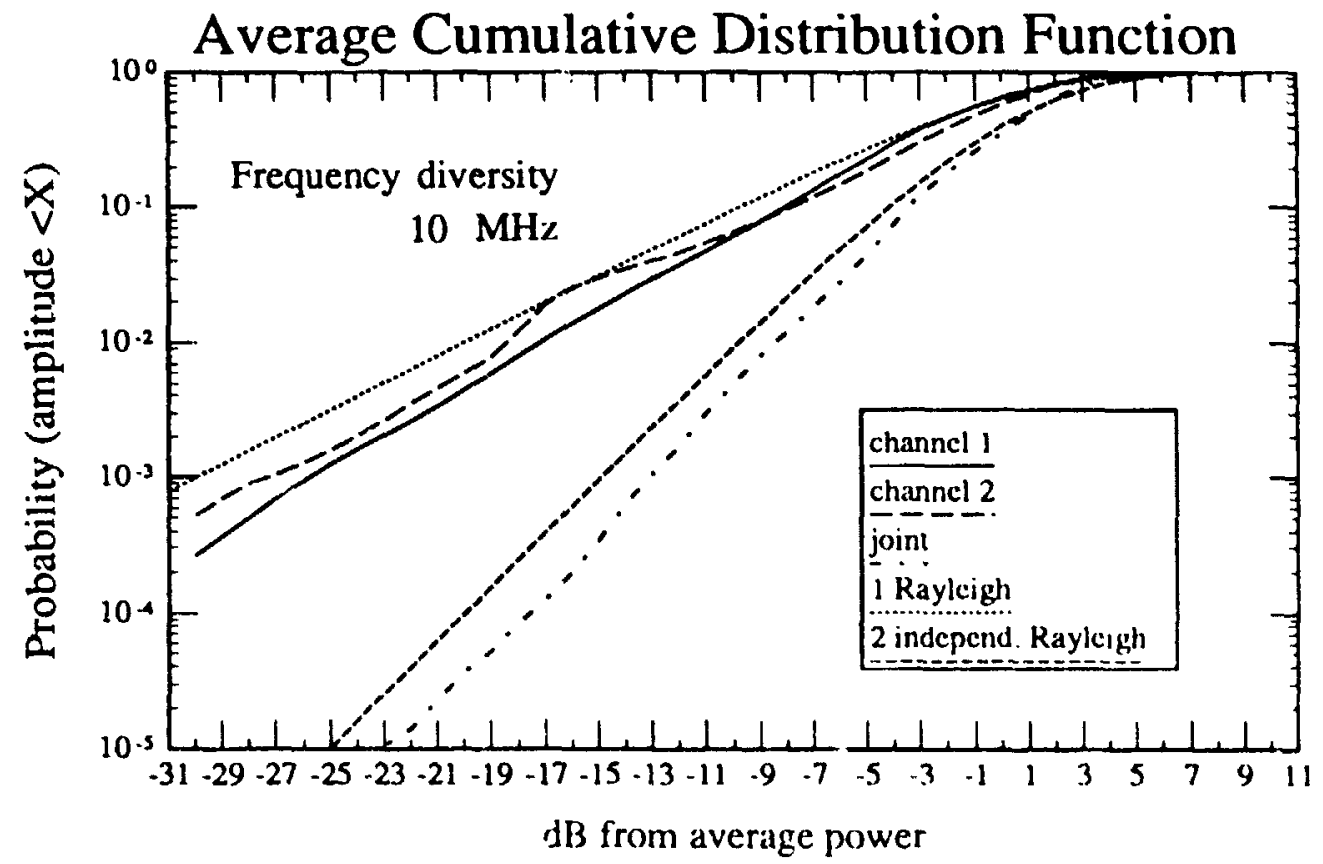

Figure- 4.8: Cumulattve distribution function for $10 \mathrm{MI/2}$ sparing 


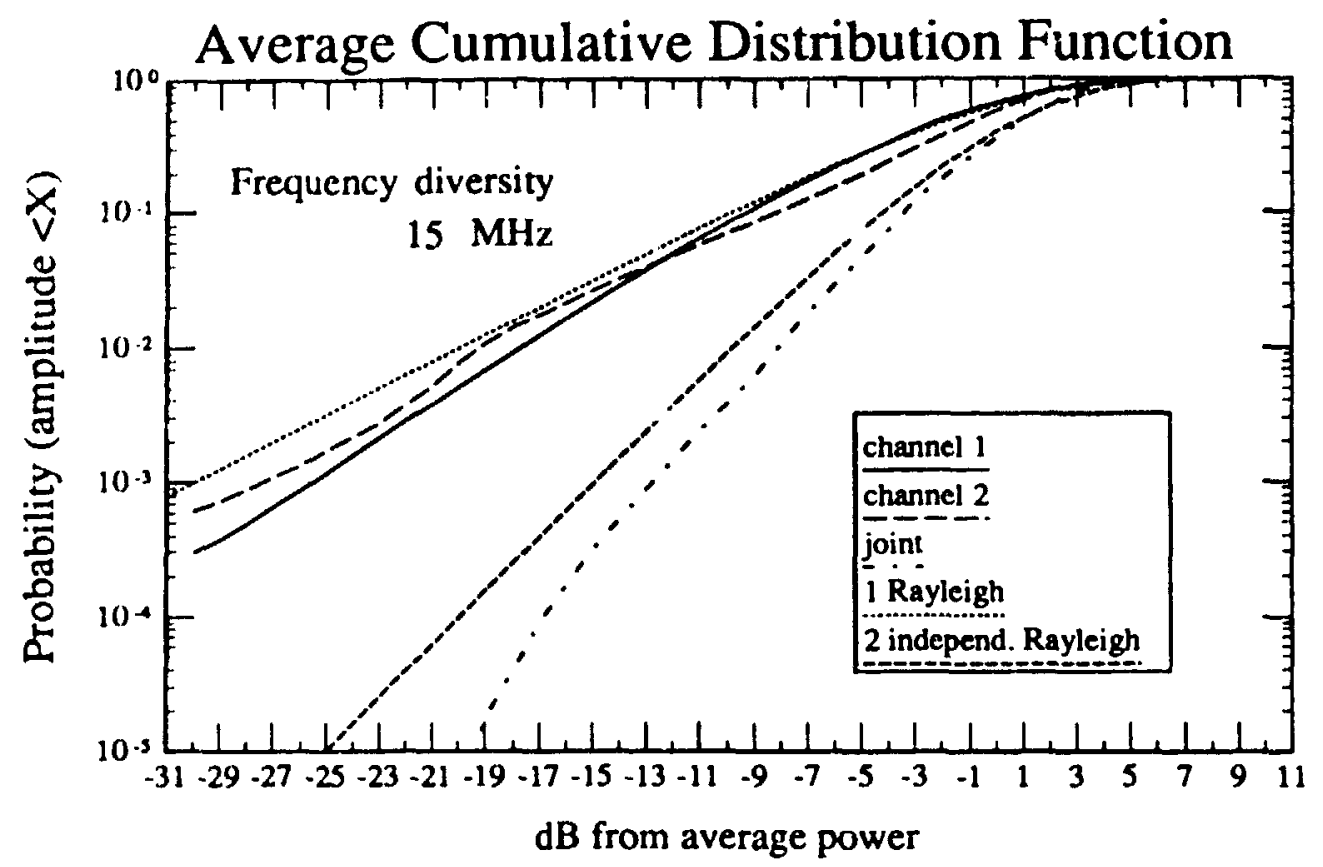

Figure- 4.9: Cumulative distribution functicn for $15 \mathrm{MHz}$ spacing

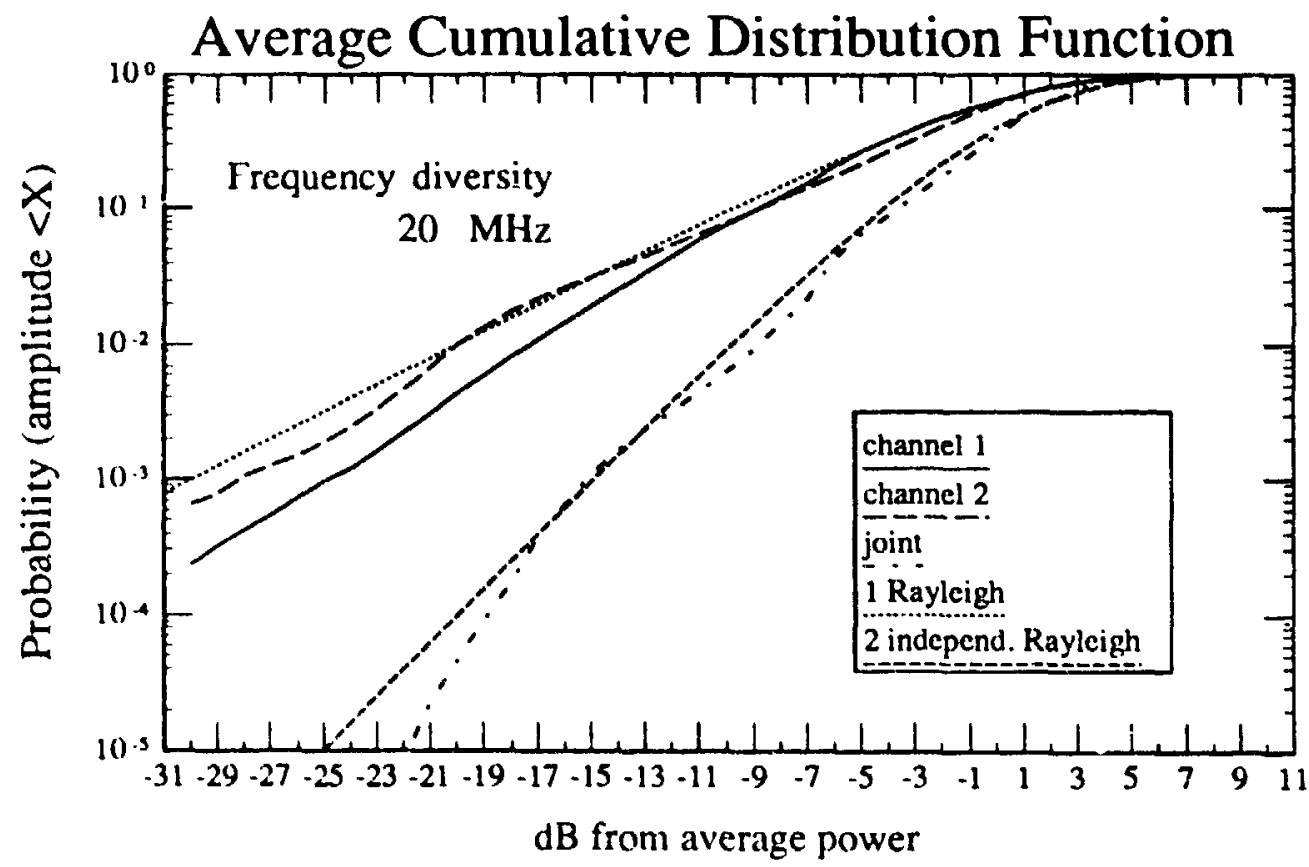

Figure- 4.10: Cumulative distribution function for $20 \mathrm{MHz}$ spacing 


\subsubsection{Space diversity}

The graphs of cumulative distribution functions for the space diversity cases are shown in figures 4.11 to 4.15 . The diversity improvement values for the five spacings are displayed in table 4.2. By examining the table, we can see that, contrary to the frequency diversity case, the improvement is not increasing with separation. At the $5 \%$, $1 \%$ and $.5 \%$ probability level, all the improvement values are basically the same. Their range is again very tight: within $1.2,1.2$ and $.8 \mathrm{~dB}$ respectively from each other and within $1.1, .9$ and .8 from the Rayleigh value. The range of improvement values for $.1 \%$ is again much larger. The explanation to this is also the same: the small number on samples reducing their value as accurate representation of the the received signal's statistics. These results indicate that there is no advantage to separate the antennas by more than $.75 \lambda$. This is in agreement with theory [12] and previous experiments [25].

\begin{tabular}{|c|c|c|c|c|}
\hline \multicolumn{5}{|c|}{ SPACE IMPROVEMENT TABLE } \\
\hline $\begin{array}{c}\text { wave } \\
\text { length }\end{array}$ & $5 \%$ Level & $1 \%$ Level & $.5 \%$ Level & $.1 \%$ Level \\
\hline .75 & 7.0 & 10.5 & 11.8 & 12.5 \\
\hline 1 & 7.1 & 10.1 & 11.6 & 16.3 \\
\hline 1.5 & 6.4 & 9.9 & 11.3 & 15.7 \\
\hline 1.66 & 6.0 & 9.5 & 11.0 & 14.8 \\
\hline 2 & 5.8 & 9.3 & 11.0 & 16.6 \\
\hline \hline Rayleigh & 6.9 & 10.2 & 11.8 & 15 \\
\hline & \multicolumn{5}{|c|}{ all values in $\mathrm{dB}$} \\
\hline
\end{tabular}

Table 4.2 Space diversity improvement valucs 


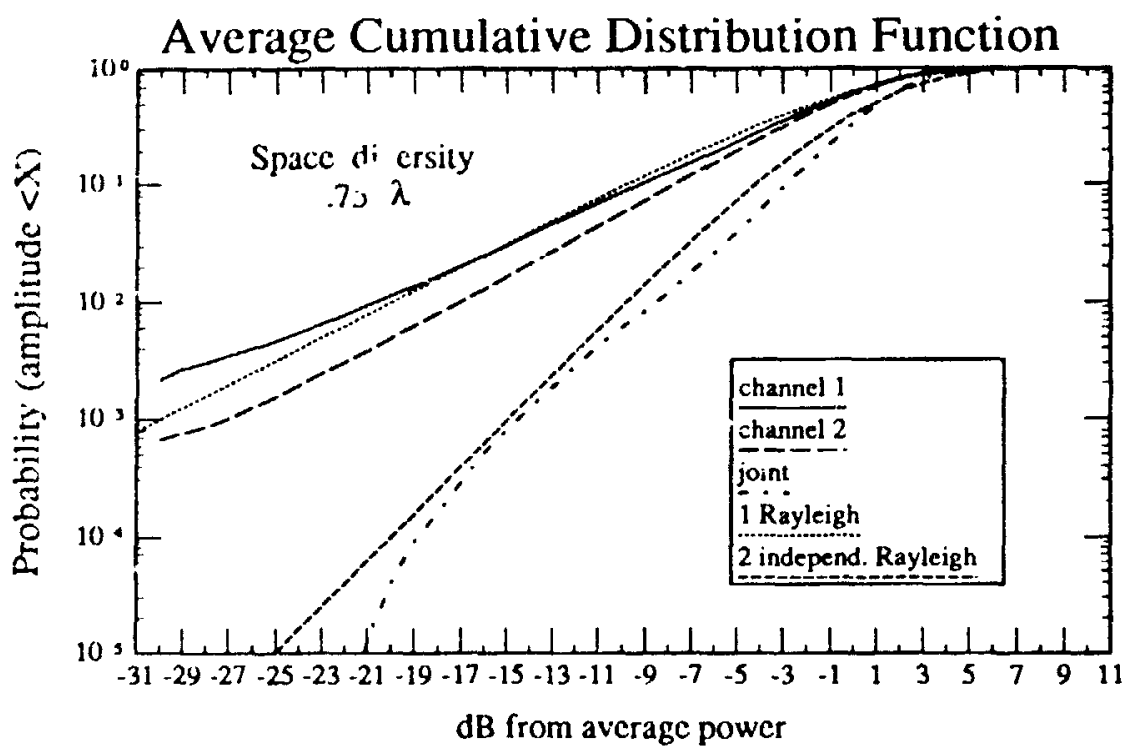

Figure- 4.11: Cumulative distribution function for .75 $\lambda$ spacing

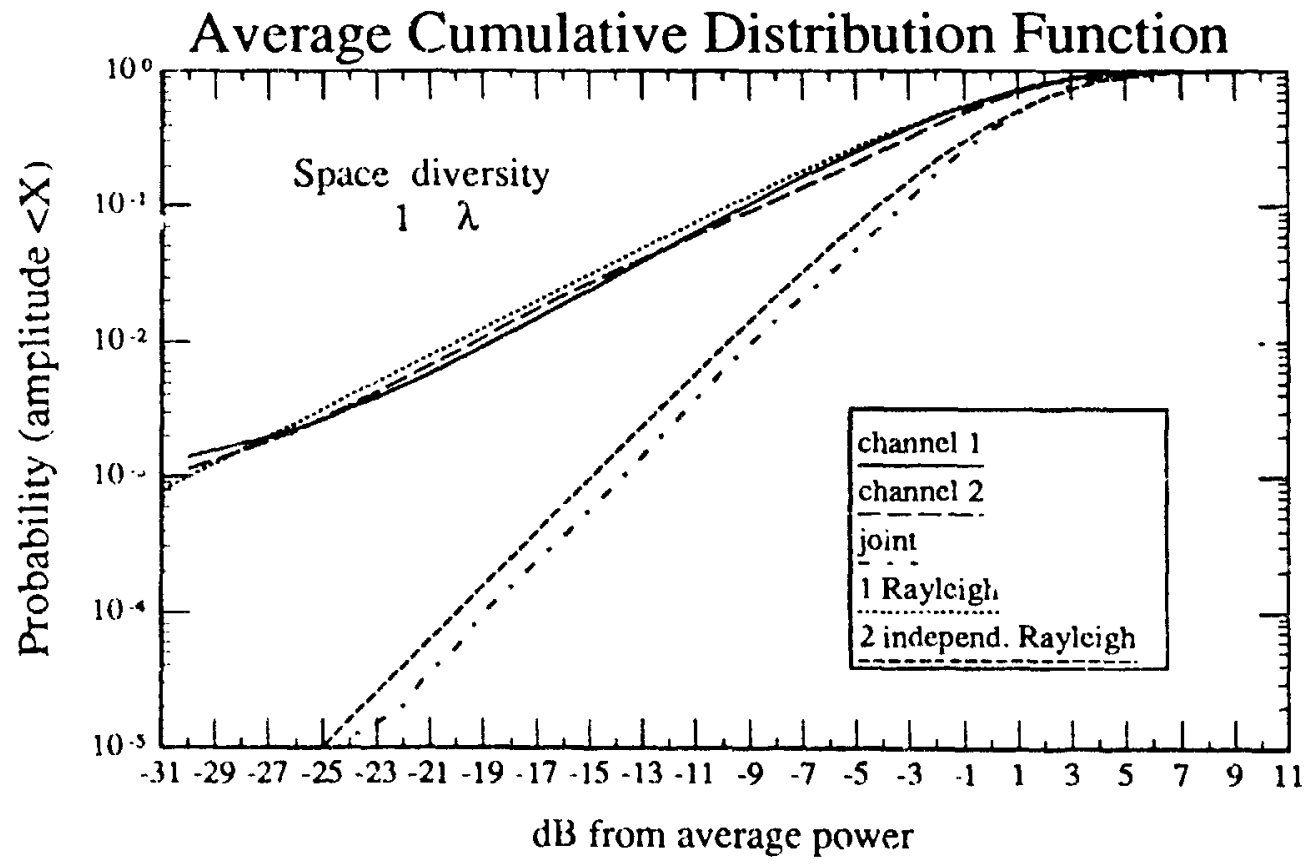

Figure- 4.12: Cumulative disiribution function for $1 \lambda$ spacing 


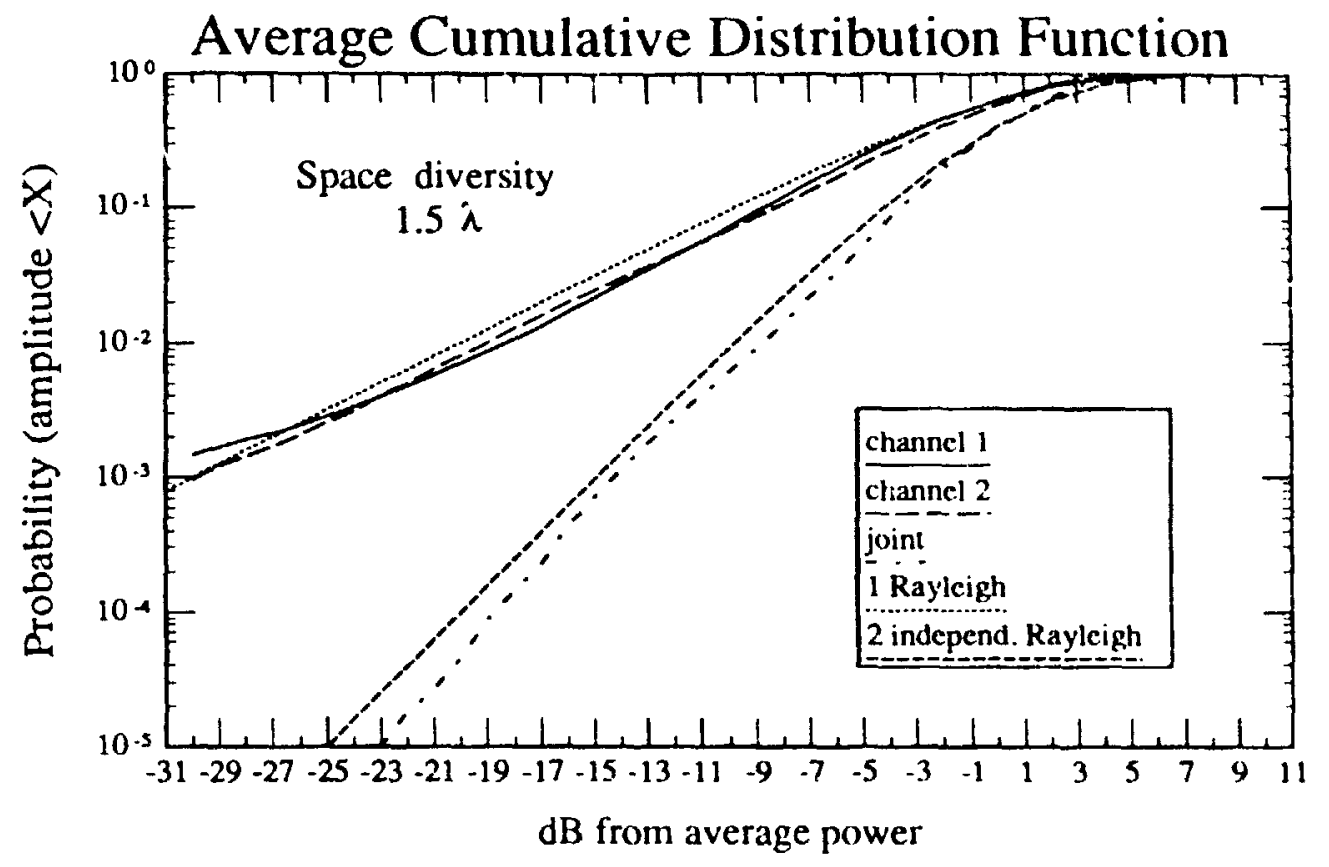

Figure- 4.13: Cumulative distribution function for $1.5 \lambda$ spacing

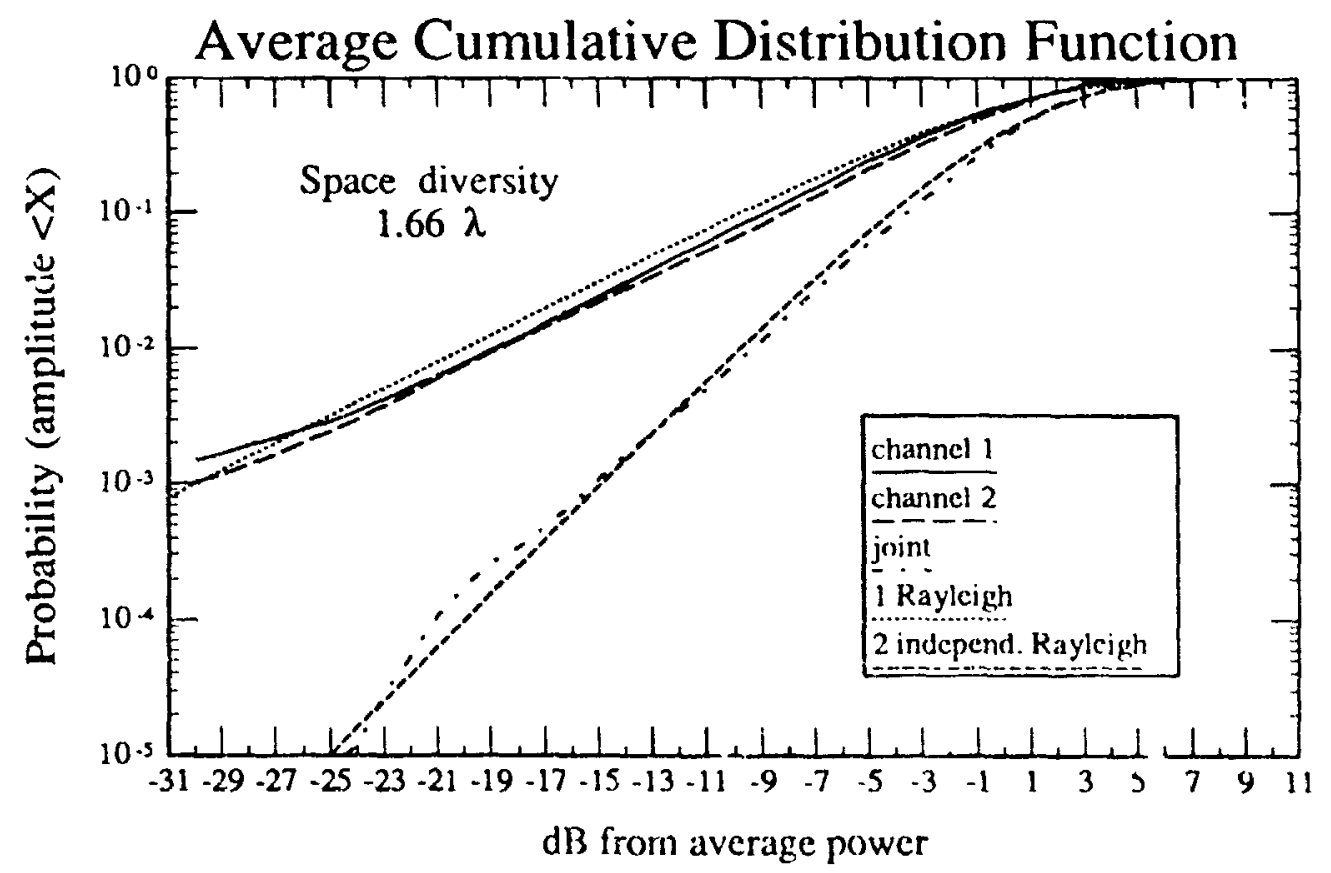

Figure- 4.14: Cumulative distribution funcuon for $1.60 \lambda$ spacting 


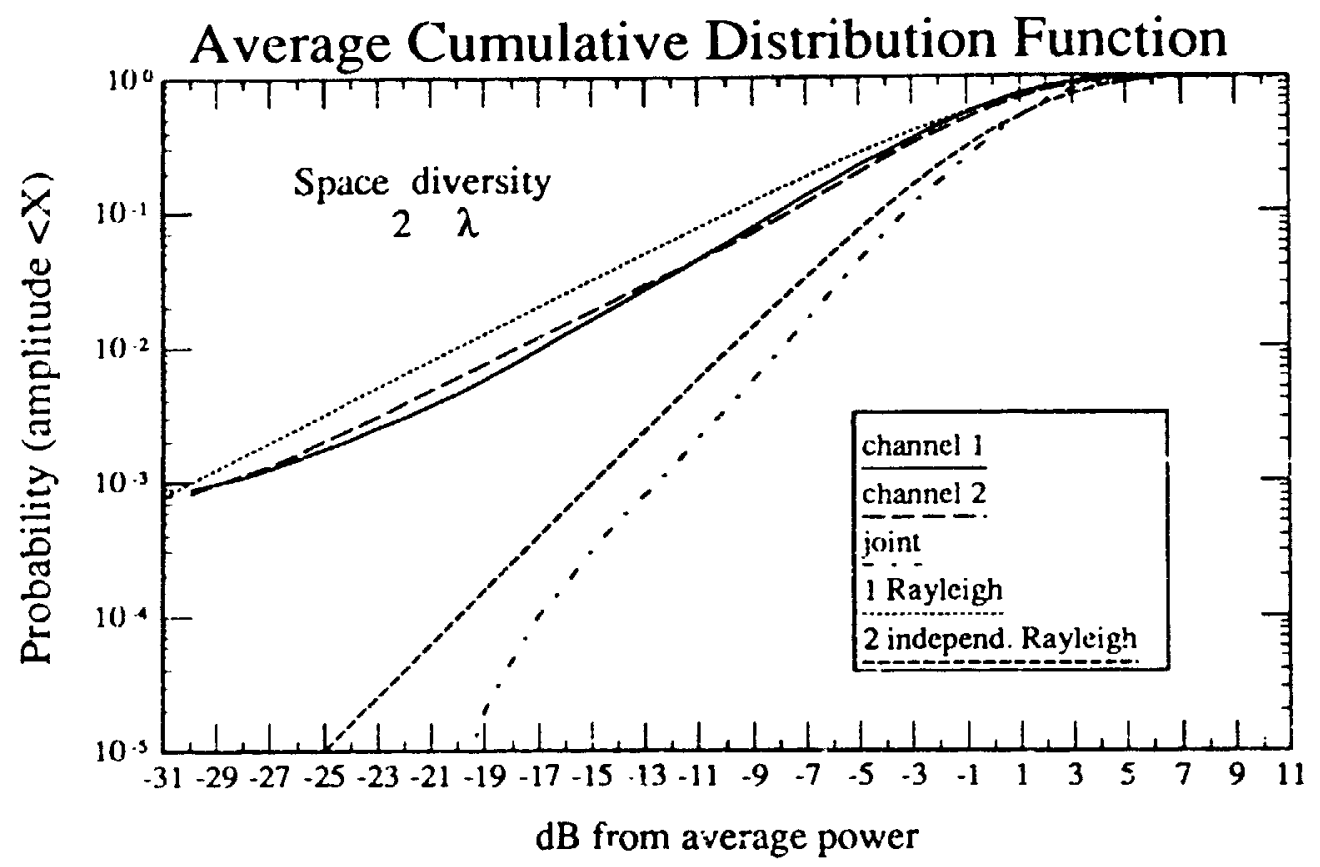

Figure- 4.15: Cumulative distribution function for $2 \lambda$ spacing

\subsubsection{Polarization diversity}

The graph of cumulative distribution functions for polarization diversity is shown in figure 4.16 . The diversity improvement values for level $5 \%$ to $.1 \%$ are displayed in table 4.3. It is interesting to see that polarization diversity yields an improvement over no diversity. The improvement values are lower than for frequency and space diversity but they are still significant. The lower value would indicate a higher correlation between each channel. These values were obtained from cumulative distributions calculated assuming the same average received power in both channels. The horizontal antenna has a $3 \mathrm{~dB}$ disadvantage due to the nulis in its radiation pattern (see section 2.2 ). With an antenna design without this inconvenience, full advantage could be taken of these improvements.

Although great care was taken to have the two antennas very close, it is possible that part of the improvement could be due to a space diversity effect. It can be seen in plate 6 that the antenna were not exactly at the same iocation. The two radiating clements were separated by about $1 \mathrm{~cm}(\approx \lambda / 30)$ at their center point. 


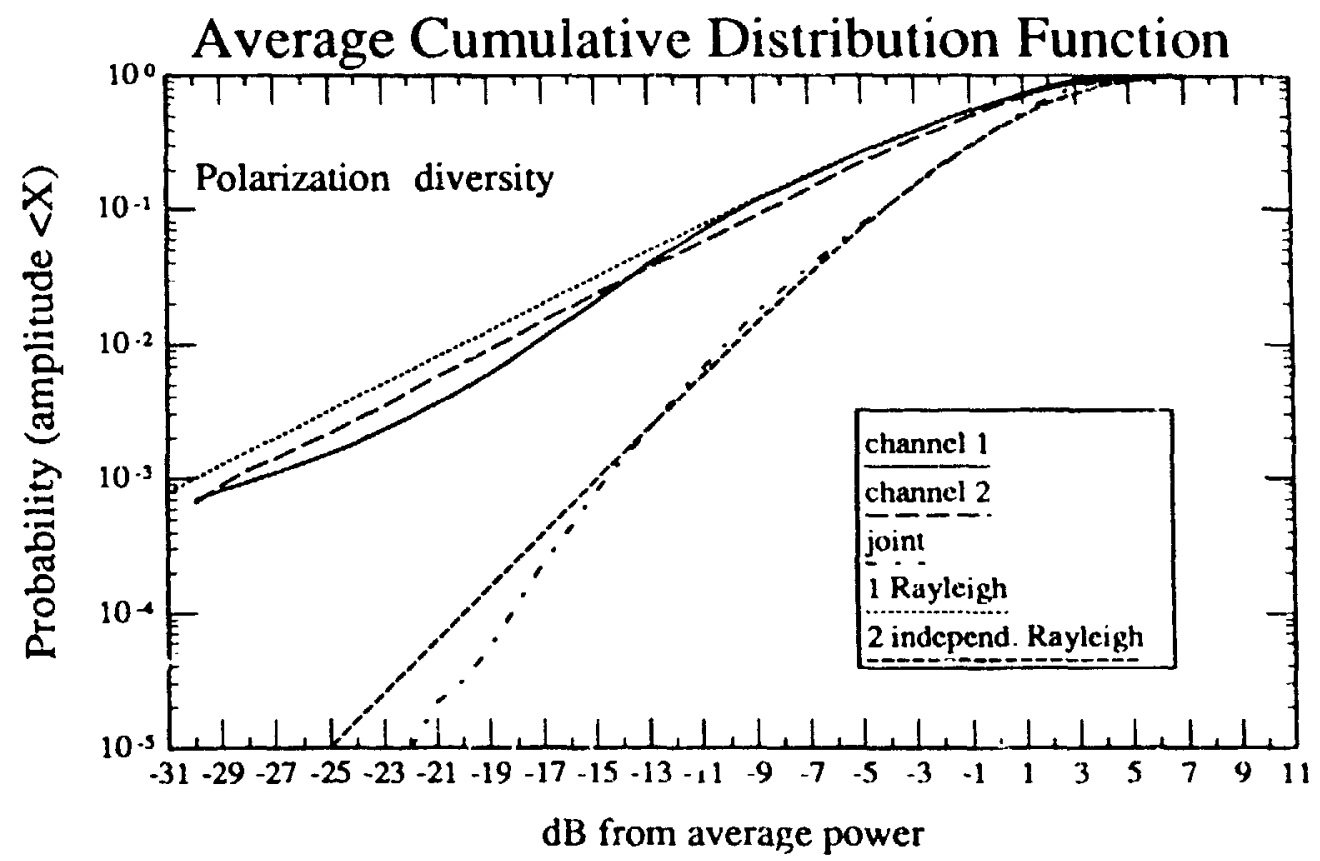

Figure- 4.16: Cumulative distribution function for $90^{\circ}$ spacing

\begin{tabular}{|c|c|c|c|c|}
\hline \multicolumn{5}{|c|}{ POLARIZATION IMPROVEMENT TABLE } \\
\hline 90 & 5\% Level & 1\% Level & $\mathbf{5 \%}$ Level & $\mathbf{. 1 \%}$ Level \\
degrees & 5.8 & 7.8 & 9.0 & 13.5 \\
\hline \hline Rayleigh & 6.9 & 10.2 & 11.8 & 15 \\
\hline & \multicolumn{4}{|c|}{ all values in $\mathrm{dB}$} \\
\hline
\end{tabular}

Tabie 4.3 Polarization diversity improvement 


\subsection{Atten'uation measurements}

The values of the exponenis in the distance-power law calculated from the scatter diagrams, as explained in section 4.2 .4 , are listed in table 4.4. Figures $4.17,4.18$ and 4.19 show three diagrams as examples for each diversity type. The remainder of the diagrams (8) are in appendix D. Except for one value in channel 1 (4.13, for spacing of $1.5 \lambda$ ), they all are between 3.17 and 3.71. The averages values are 3.62 and 3.49 for channel 1 and channel 2 respectively. If we remove the high value in channel 1 , its average becomes 3.56 , even closer to channel 2 's.

\begin{tabular}{|c|c|c|c|c|}
\hline & SCATTER DIAGRAM GRADIENT & correlation \\
\hline & channel 1 & channel 2 & $\begin{array}{c}\text { coeff } \\
\text { channel 1 }\end{array}$ & $\begin{array}{c}\text { coeff } \\
\text { channel 2 }\end{array}$ \\
\hline $1 \mathrm{MHz}$ & 3.69 & 3.60 & .945 & .942 \\
\hline $\mathbf{5} \mathbf{~ M H z}$ & 3.60 & 3.47 & .959 & .928 \\
\hline $10 \mathrm{MHz}$ & 3.65 & 3.43 & .940 & .940 \\
\hline $15 \mathrm{MHz}$ & 3.71 & 3.54 & .929 & .925 \\
\hline $20 \mathrm{MHz}$ & 3.67 & 3.59 & .940 & .908 \\
\hline .75 wavel. & 3.49 & 3.53 & .971 & .967 \\
\hline 1 wavel. & 3.43 & 3.51 & .973 & .965 \\
\hline 1.5 wavel. & 4.13 & 3.47 & .982 & .966 \\
\hline 1.66 wavel. & 3.34 & 3.17 & .947 & .941 \\
\hline 2 wavel. & 3.65 & 3.48 & .972 & .958 \\
\hline polarization & 3.40 & 3.56 & .942 & .958 \\
\hline average & 3.62 & 3.49 & & \\
\hline
\end{tabular}

Table 4.4 Exponents of distance-power law for all diversity cases

These tw- values are in agreement with other reported distance sower law measurements [15]. In one office building with plasterboard walls, Alexander measured a gradient of 3.7. In all scatter diagrams, the data for the 20 locations was used to do 
the fitting. The floor plan and the list of locations presented in Appendix E must be used to relate the distances to the attenuation values. There was no attempt to separate the line-of-sight (LOS) locations (usually short distances) from the non-LOS locations (usually longer distances). As mentioned in [26], the attenuation rate is not the same for the two cases. The LOS locations usually give exponent values closer to the free-space value of 2 .

For frequency and space diversity, all curves (except figure D.6, space diversity, 1.5 d) are almost one over the other. This is an indication that the relative attenuation is consistently the same for both channels and that the overall rate of attenuation (distancepower law exponent) is also very close for all spacings and all frequencies.

The polarization diversity scatter diagram shows the two curves almost parallel but well separated. Channel 2's relative attenuation is consistently higher than channel I's. Channel 2 had the horizontal antenna. From this curve, the horizontal dipole has a relative attenuation about $3.4 \mathrm{~dB}$ worse than the vertical antenna. The difference is due to the non-uniform radiation pattern of an horizontal dipole, as discussed in section 2.2. The difference mentioned by Cox et al in [13] is $3 \mathrm{~dB}$.

The correlation coefficients calculated when the fitting was done are also presented in the table. These coefficients give an indication of the degree of fit of the curve. 


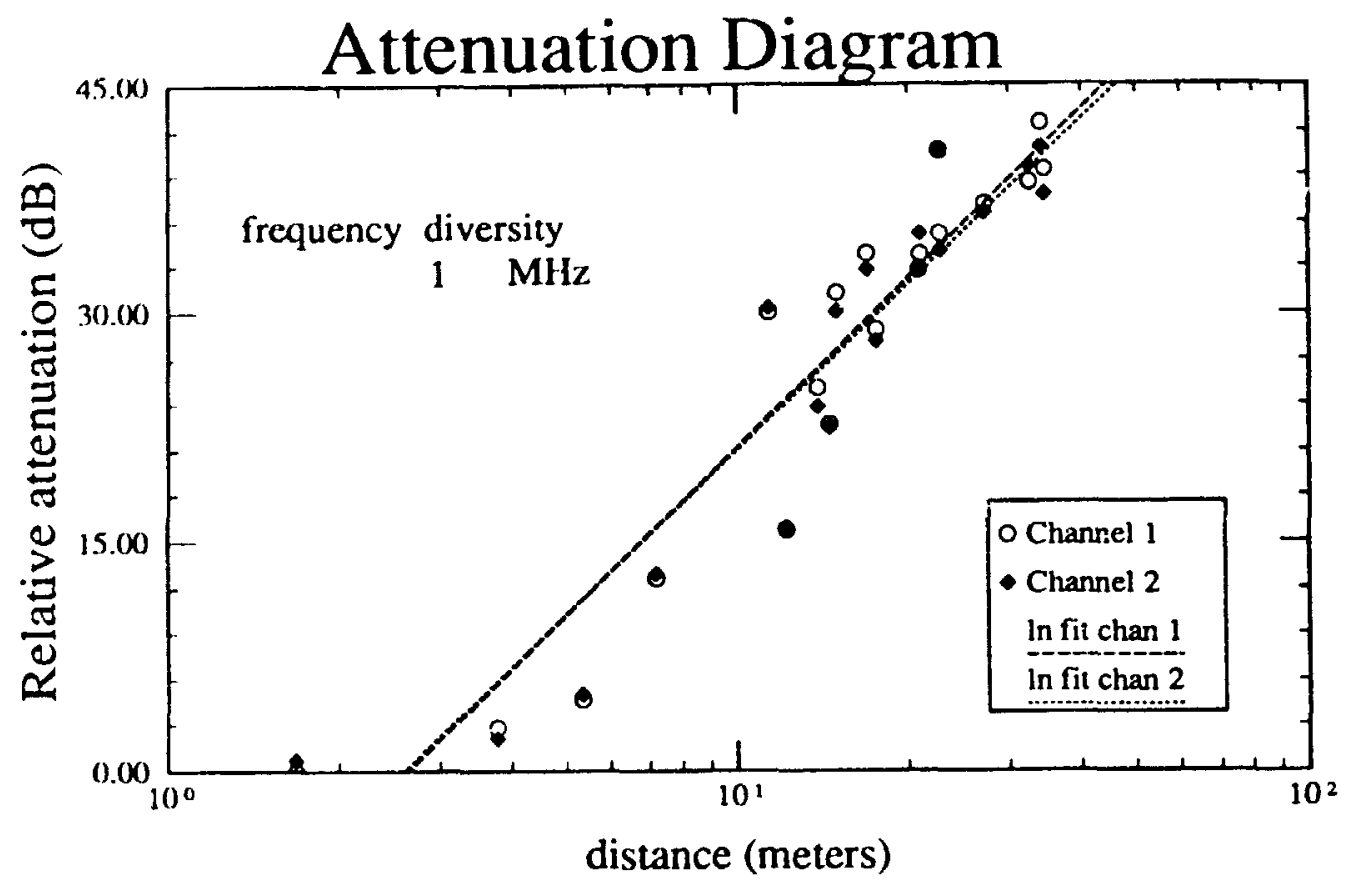

Figure- 4.17: Scatter diagram for frequency diversity $I \mathrm{MHz}$, all locations

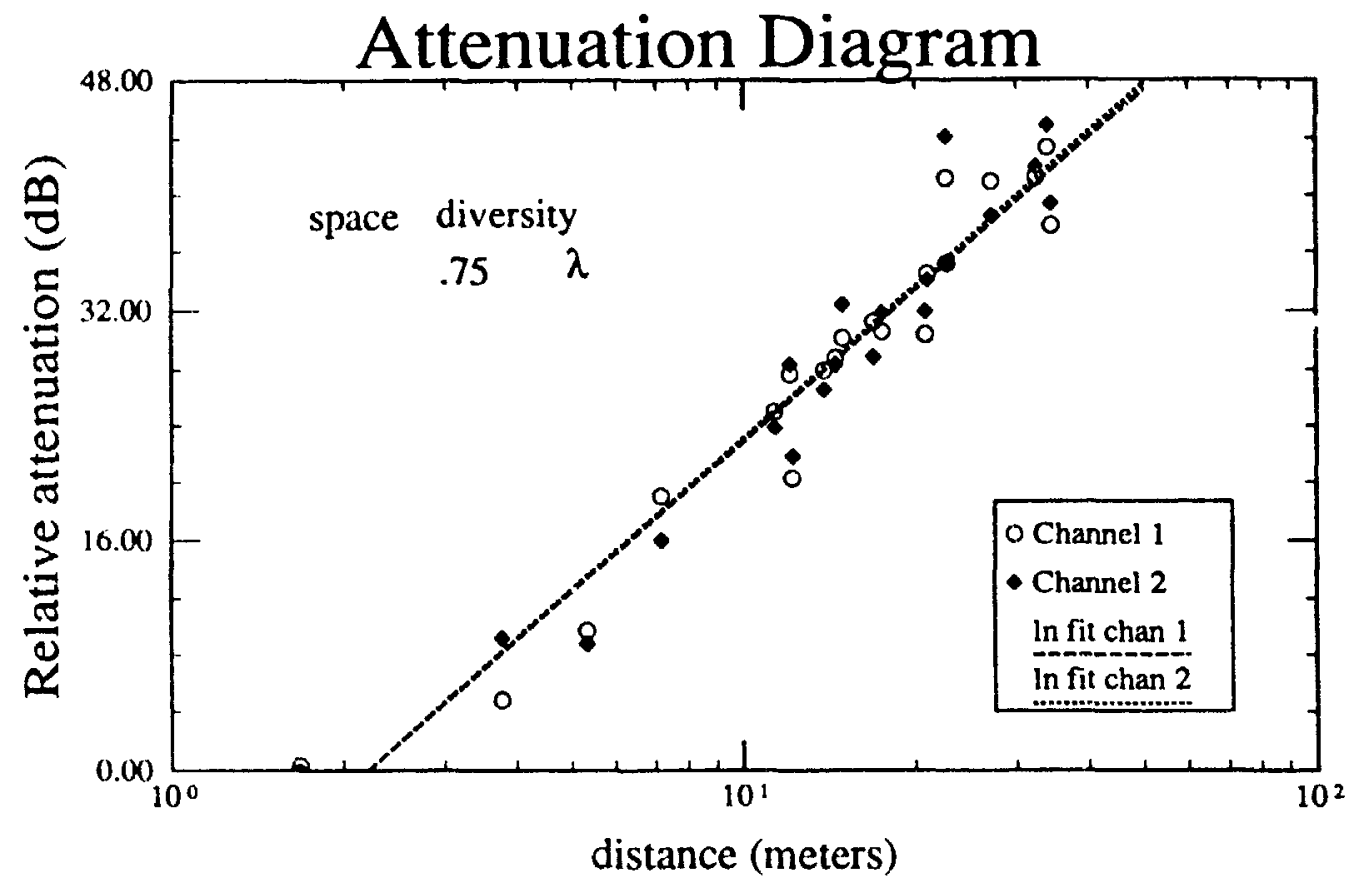

Figure- 4.18: Scalter diagram for space diversity $.75 \lambda$, all locations 


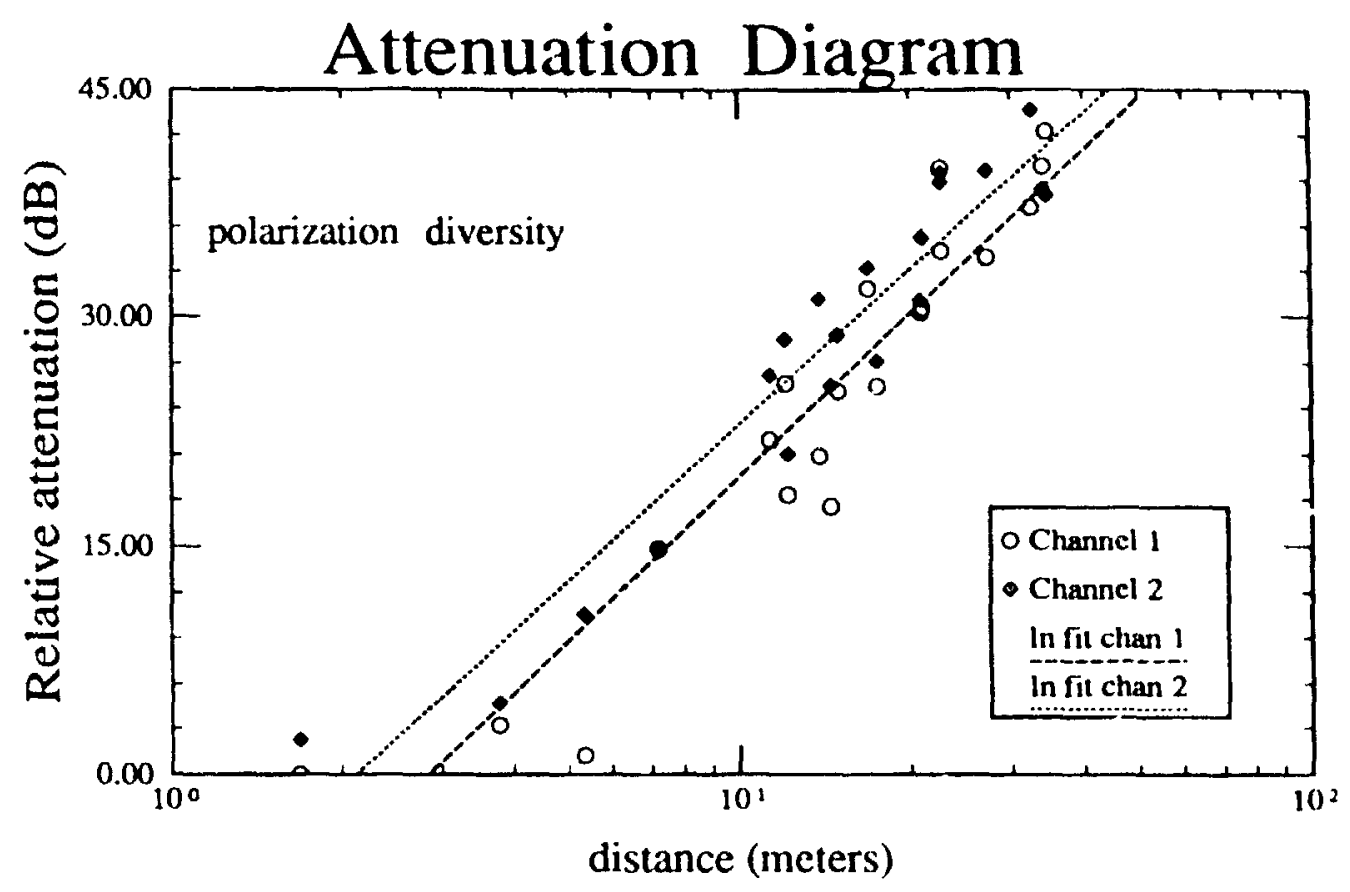

Figure- 4.19: Scatter diagram for polarization diversity, all locations

\subsection{Summary}

This section will summarize and regroup the results obtained in section 4.3 and 4.4.

For frequency diversity, at all but the $.1 \%$ level, the largest diversity improvement were achieved for spacings of 10 to $20 \mathrm{MHz}$. Lower improvement was achieved with a $5 \mathrm{MHz}$ spacing and minimal improvement was achieved with a $1 \mathrm{MHz}$ spacing. The results at the $.1 \%$ level were less definite as they were based on fewer numbers of samples.

For space diversity, the results for the $5 \%, 1 \%$ and $.5 \%$ levels indicated that all five spacings are good to get the maximum improvement. As for frequency diversity, the $.1 \%$ level results were less definite for the same reason.

The polarization diversity results showed that the two channels were partially correlated but significant improvement was measured using polarization diversity. An space diversity effect could be the cause of some of the improvement because there was still a small physical separation between the two antennas $(\approx \lambda / 30)$. 
The overall exponent of the distance-power law was 3.56 for channel 1 and 3.49 for channel 2 . These values are well within previously reported values for similar types of building construction. The attenuation results for frequency and space diversity were very consistent except for one curve. The attenuation results for polarization diversity showed that the channel with the horizontal antenna had a relative attenuation $3.4 \mathrm{~dB}$ higher than that of the vertical antenna: a normal characteristics of horizontally polarized dipole antenna. 


\section{Chapter 5}

\section{Conclusion and Recommendations}

\subsection{Conclusion}

We started by reviewing theory on the fading channel and on diversity. Currently published experiments were then reviewed in order to study proven experimental installations, procedures and results. The design and implementation of our transmitters, receivers, antennas and data acquisition system were presented and explained in detail. The analysis and data processing steps conducted to obtain our results were also explained.

Three types of diversity were used: frequency, space and polarization. Frequency and antenna spacings were used as a parameter to study diversity improvement. Polarization diversity used one horizontally and one vertically polarized dipole.

The overall results are in agreement with theoiy and previously published results. Frequency diversity results showed that maximum improvement is achieved for spacings of $10 \mathrm{MHz}$ or more. Lower improvement is achieved with a spacing of $5 \mathrm{MHz}$. As a comparison, the maximum improvement figures were within $6, .9$ and $1.7 \mathrm{~dB}$ from the improvement obtained with 2 uncorrelated Rayleigh distributions.

Space diversity results showed that any antenna spacing above $.75 \lambda$ will give the maximum improvement. This confirms theory and previously reported results which 
indicated that a spacing of $.5 \lambda$ would get the maximum improvement. The improvement figures obtained were within $1.1, .9$ and $.8 \mathrm{~dB}$ from the improvement obtained with 2 uncorrelated Rayleigh distributions..

Polarization diversity results showed that significant improvement is achieved. The improvement obtained ranged from $5.8 \mathrm{~dB}$ at the $5 \%$ level to $13.5 \mathrm{~dB}$ at the $.1 \%$ level. The lower value of the improvement (cor. pared to frequency and space diversity) would mean that our two channels were somewhat correlated. The physical separation of the two antennas might have had a small space diversity effect.

Attenuation results in the form of the distance-power law's exponents were calculated using data from all 20 locations. The average value of the distance-power law's exponent was 3.49 and 3.56. This matches previously reported results for similar types of building construction. The scatter diagram for polarization diversity showed that overall attenuation for the horizontal dipole was $3.4 \mathrm{~dB}$ higher than for the vertical one, as expected for that type of anterna.

\subsection{Contribution}

The indoor radio channel is still a new channel. Some results are reported in the literature about attenuation, the type of envelope level distribution and difference between static and dynamic measurements. Except for one case of space diversity, there were no direct results on the use and effectiveness of diversity in the indoor channel. This thesis provides such results on diversity and the relative merit of frequency, space and polarization diversity. Results on frequency diversity showed what minimum spacing is good to get the most improvement for that type of building construction. It was shown that polarization diversity could be used effectively as method to obtain a second partially uncorrelated channel.

\subsection{Recommendations for future research}

The raw data collected in the course of this work was gathered in such a way as to allow a great flexibility in studying other aspects of the indoor portable channel. 
Some analysis on the distribution of fade durations and time between fides could be performed. Studies on the difference between the attenuation and envelope distribution characteristics of LOS and non-LOS locations could be done. As done in many previous work, the cumulative distribution or the probability density function could be studied to find out more precisely what type they match (e.g. Rayleigh, Rician or log-normal).

Since the combining was done by a software algorithm, other combining method such as equal gain, maximal ratio or switched combining could be easily implemented. The data gathered could be used to refine or produce a channel model. Channel error performance could be calculated for different types of modulation to see their relative performance in this environment. 


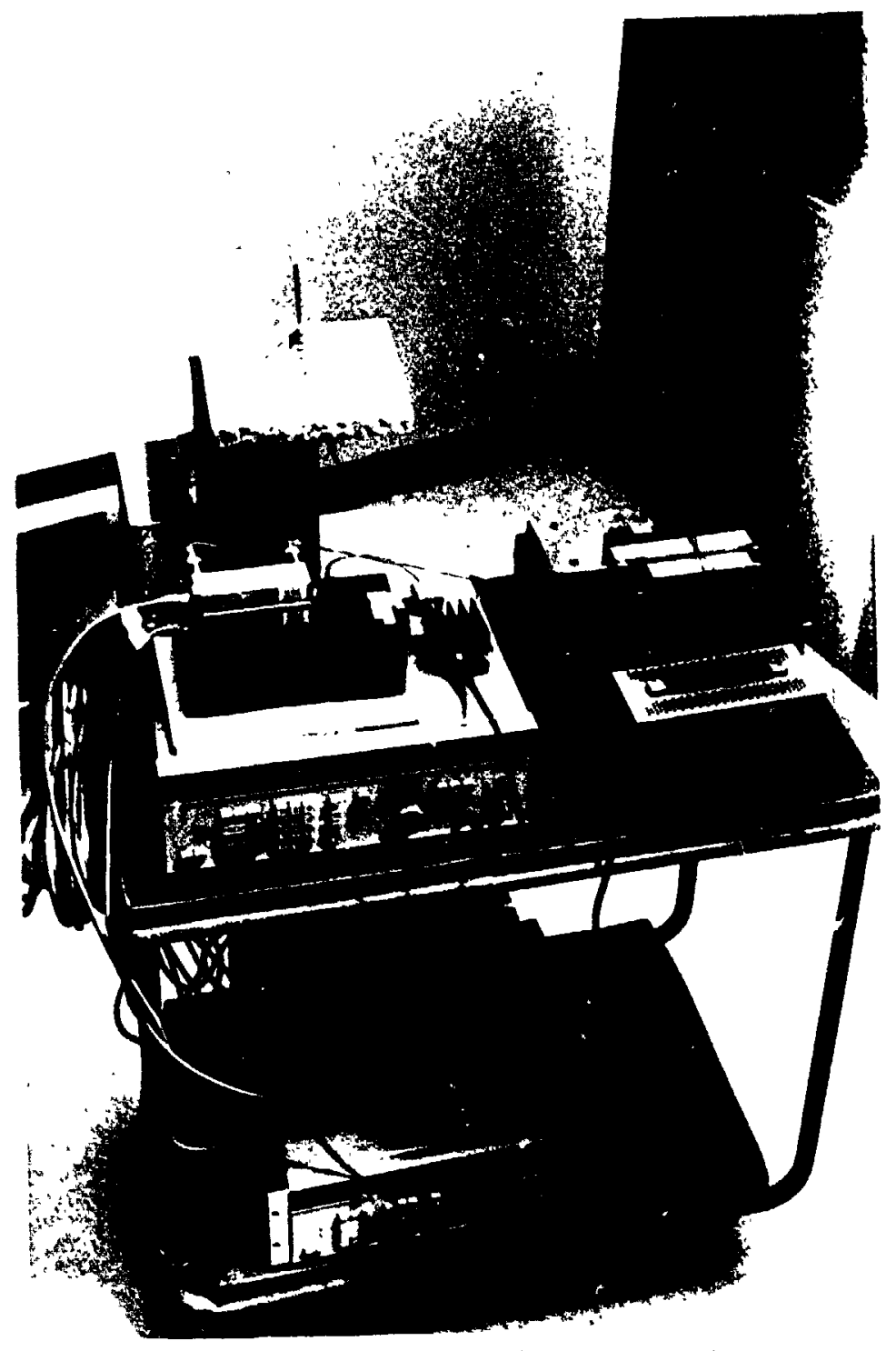

Plate 1 Frequency diversity transmitter 


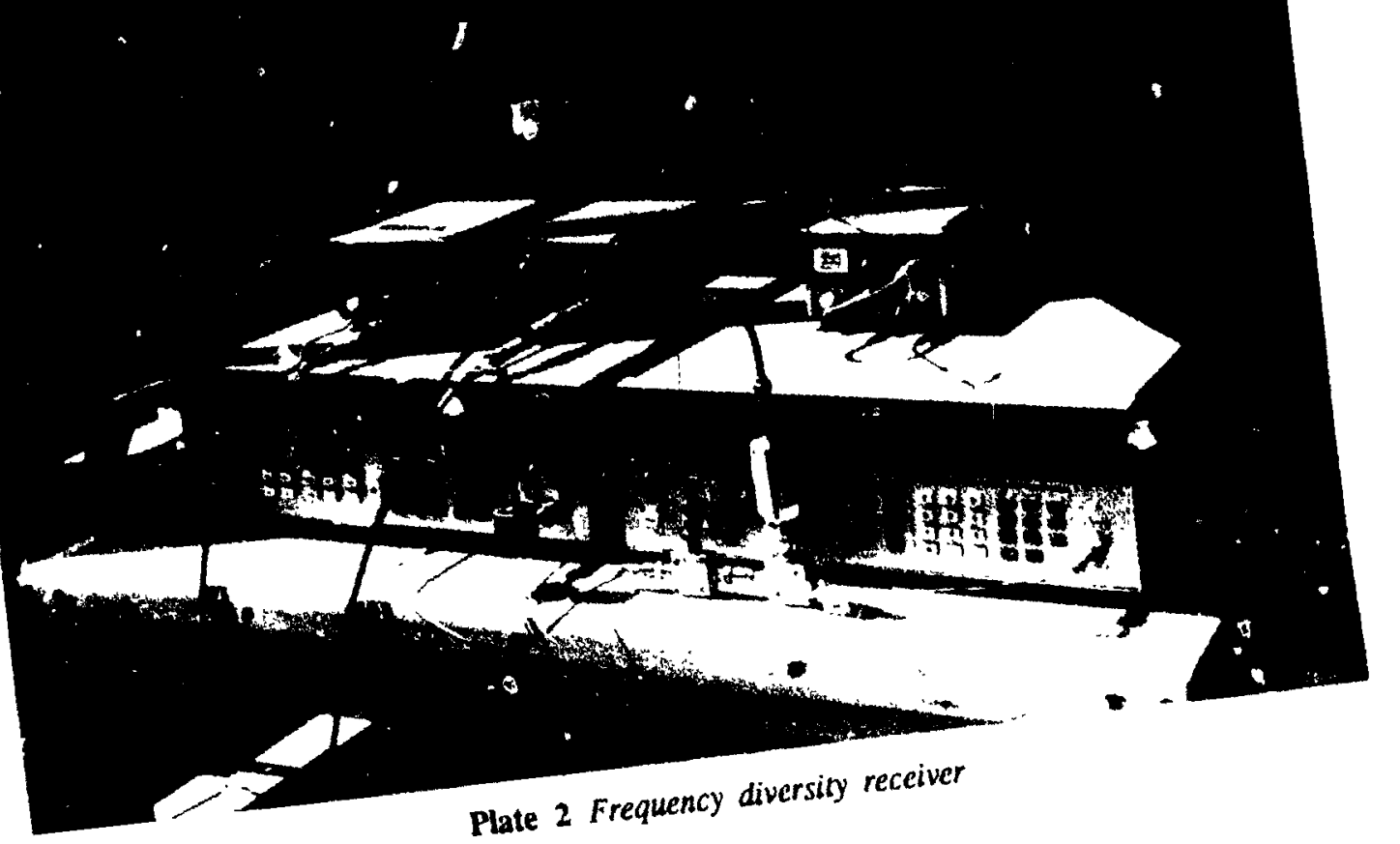



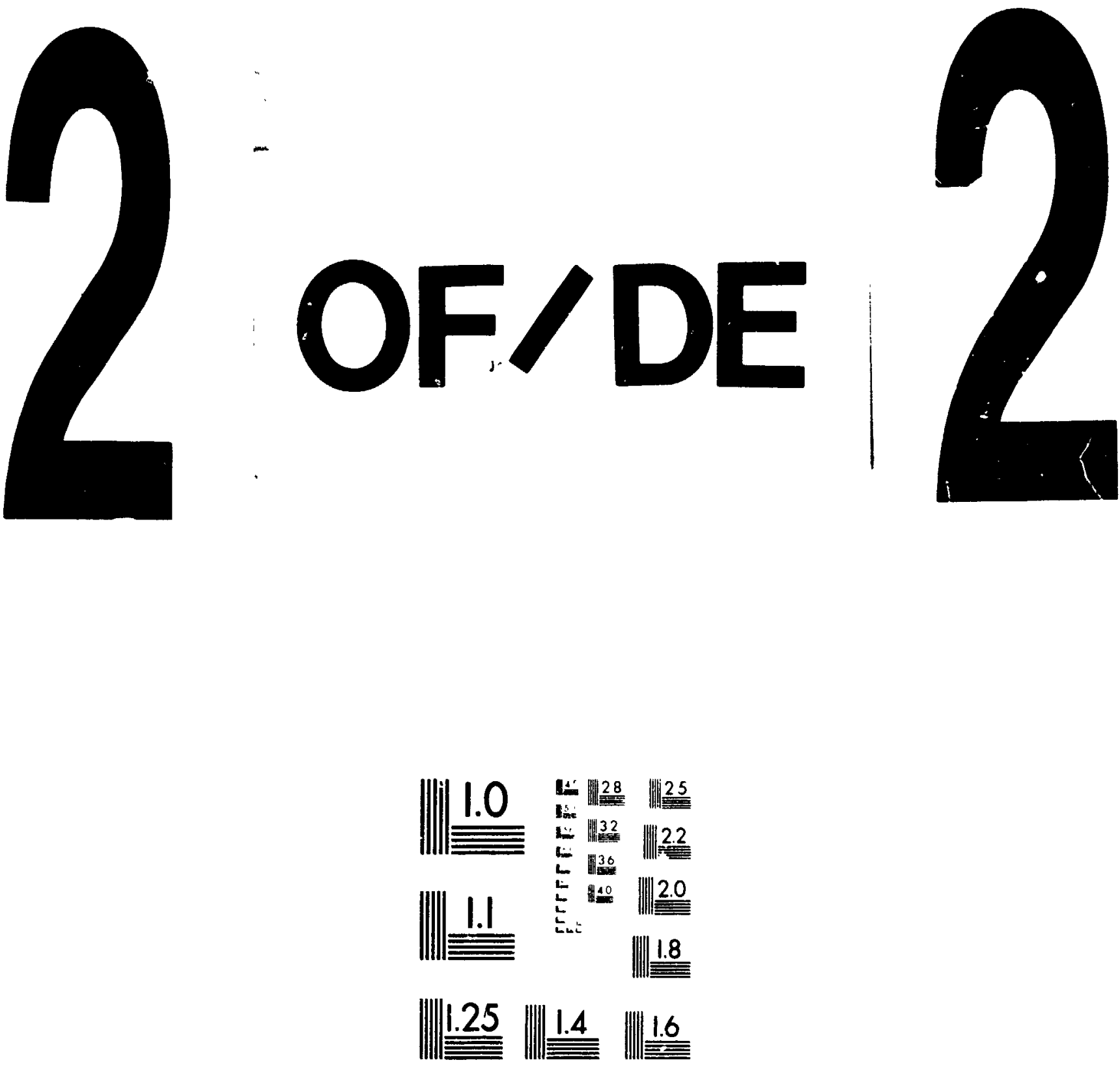

MICROCOPY RESOLUTION TEST CHART NATIONAL BUREAU OF STANDARDS STANDARD REFERENCE MATERIAL 1010a (ANSI and ISO TEST CHART NO 21 


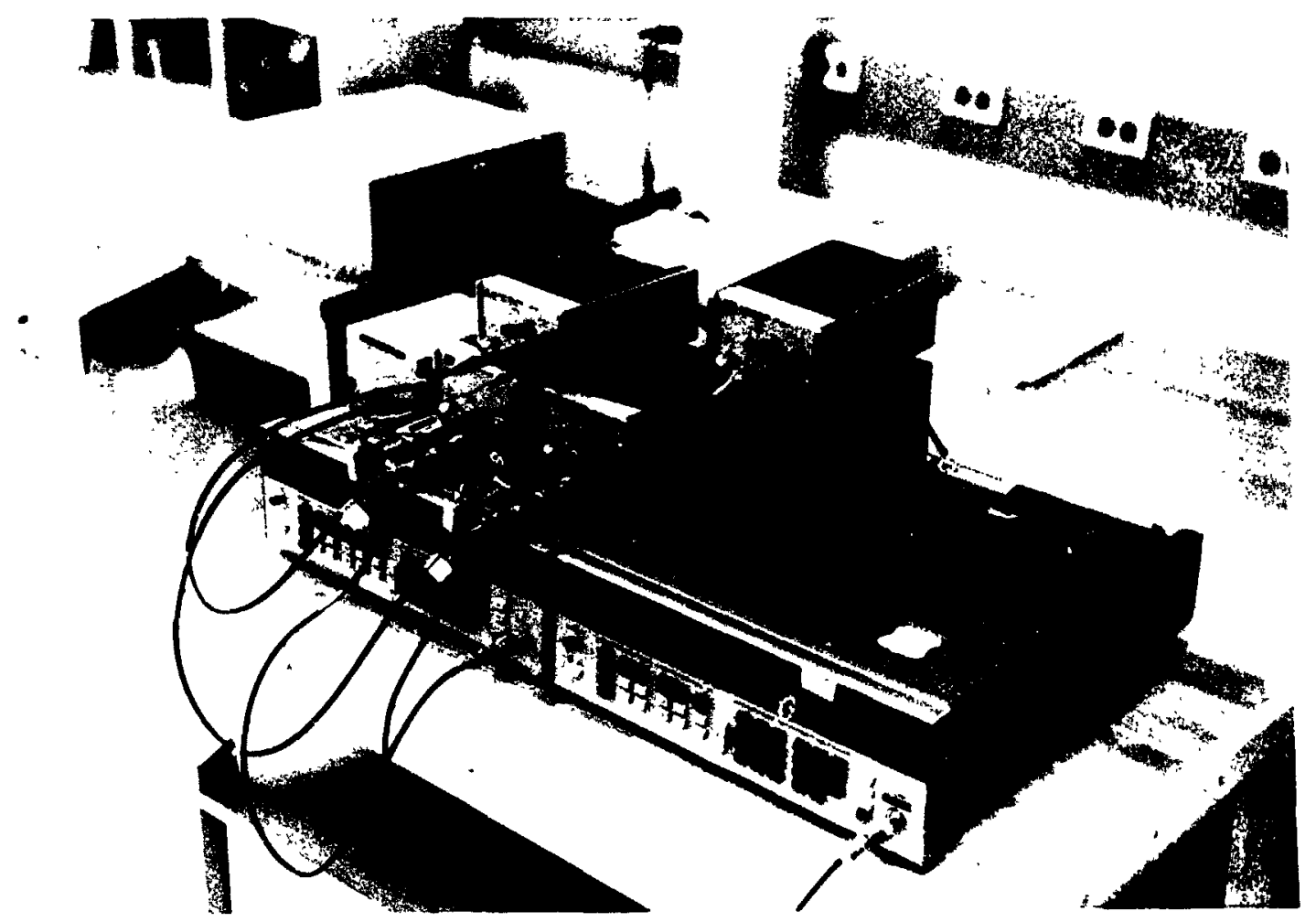

Plate 3 Space and polarization diversity receiver 


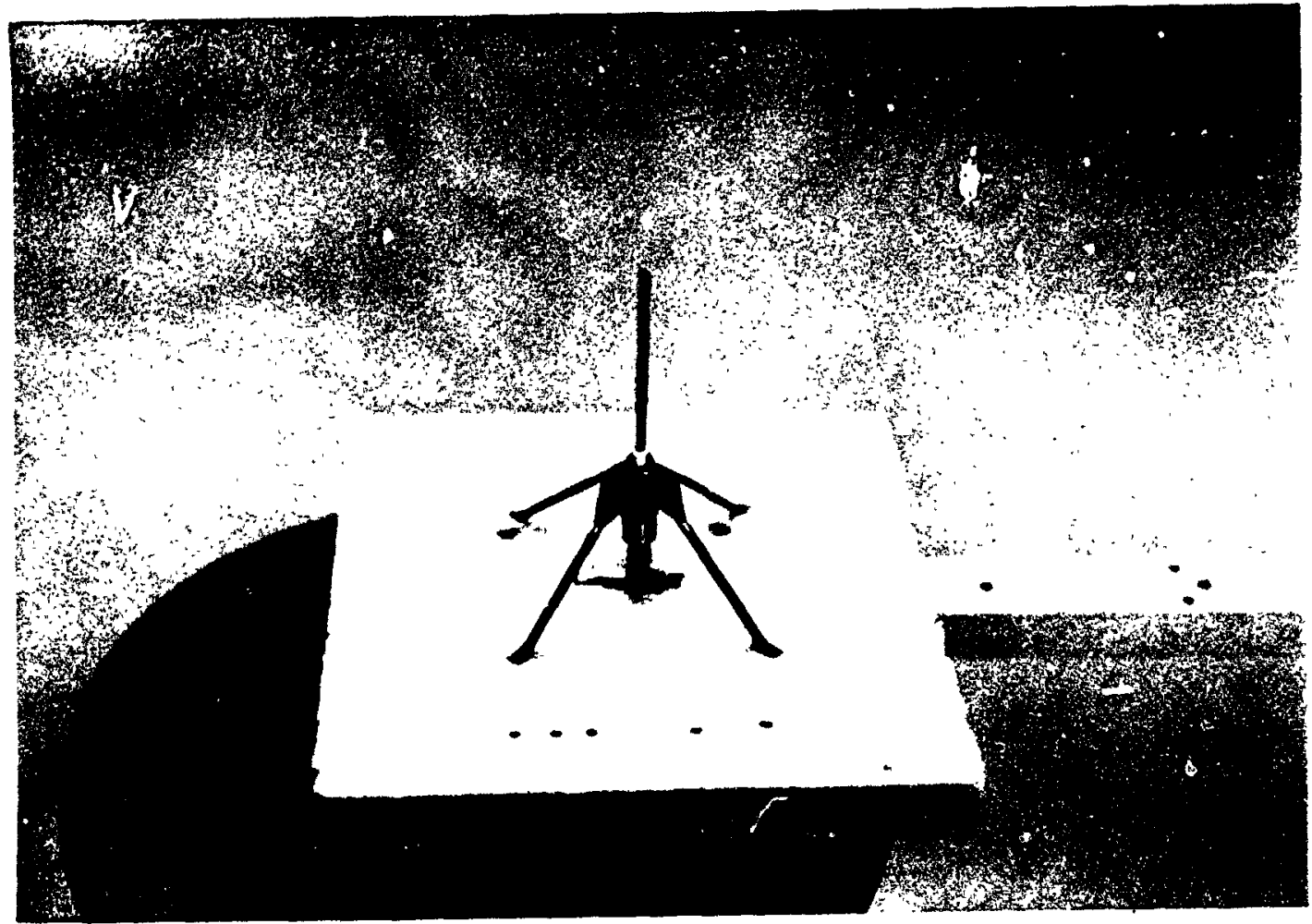

Plate 4 Example of antenna used 


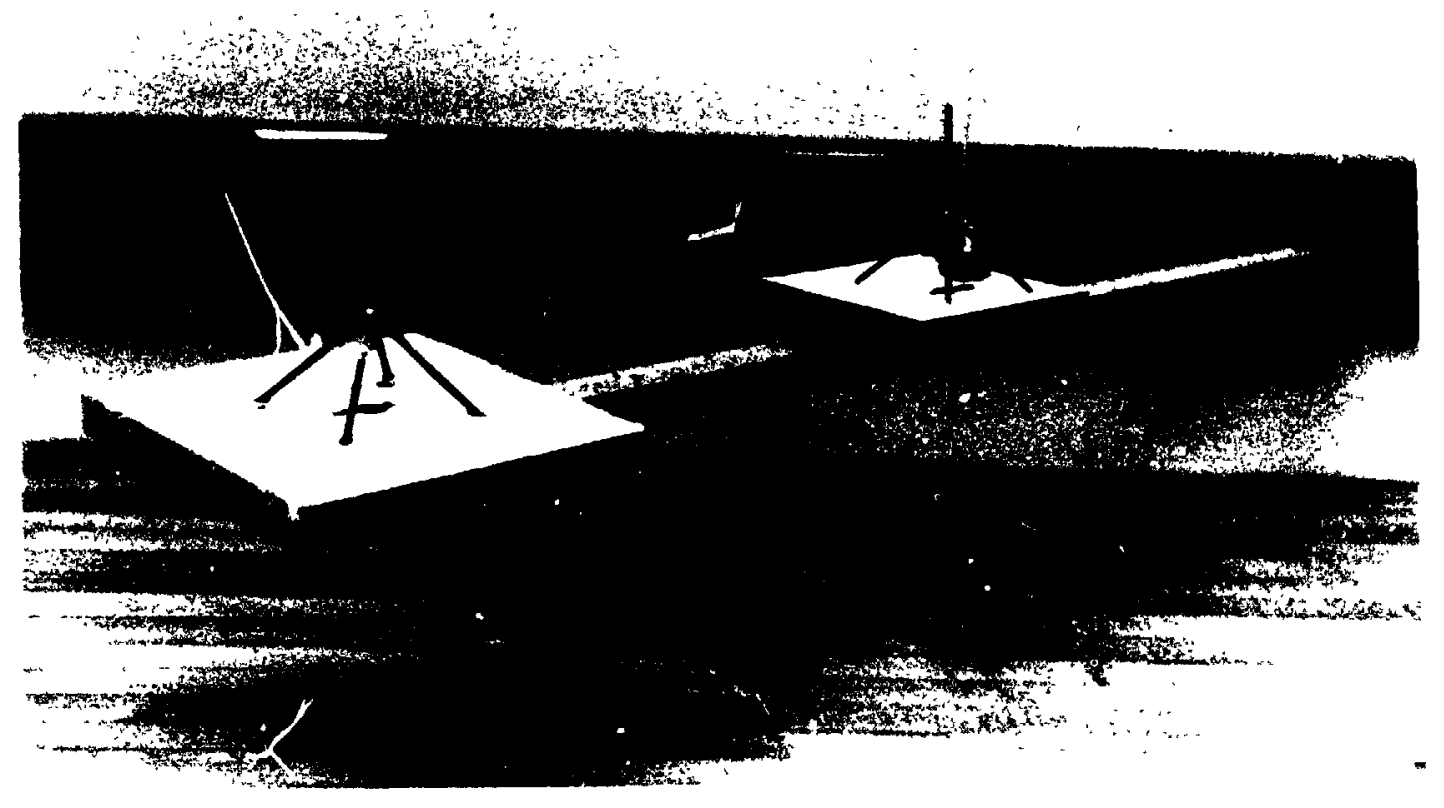

Plate 5 Holding frame for space diversity antenna 


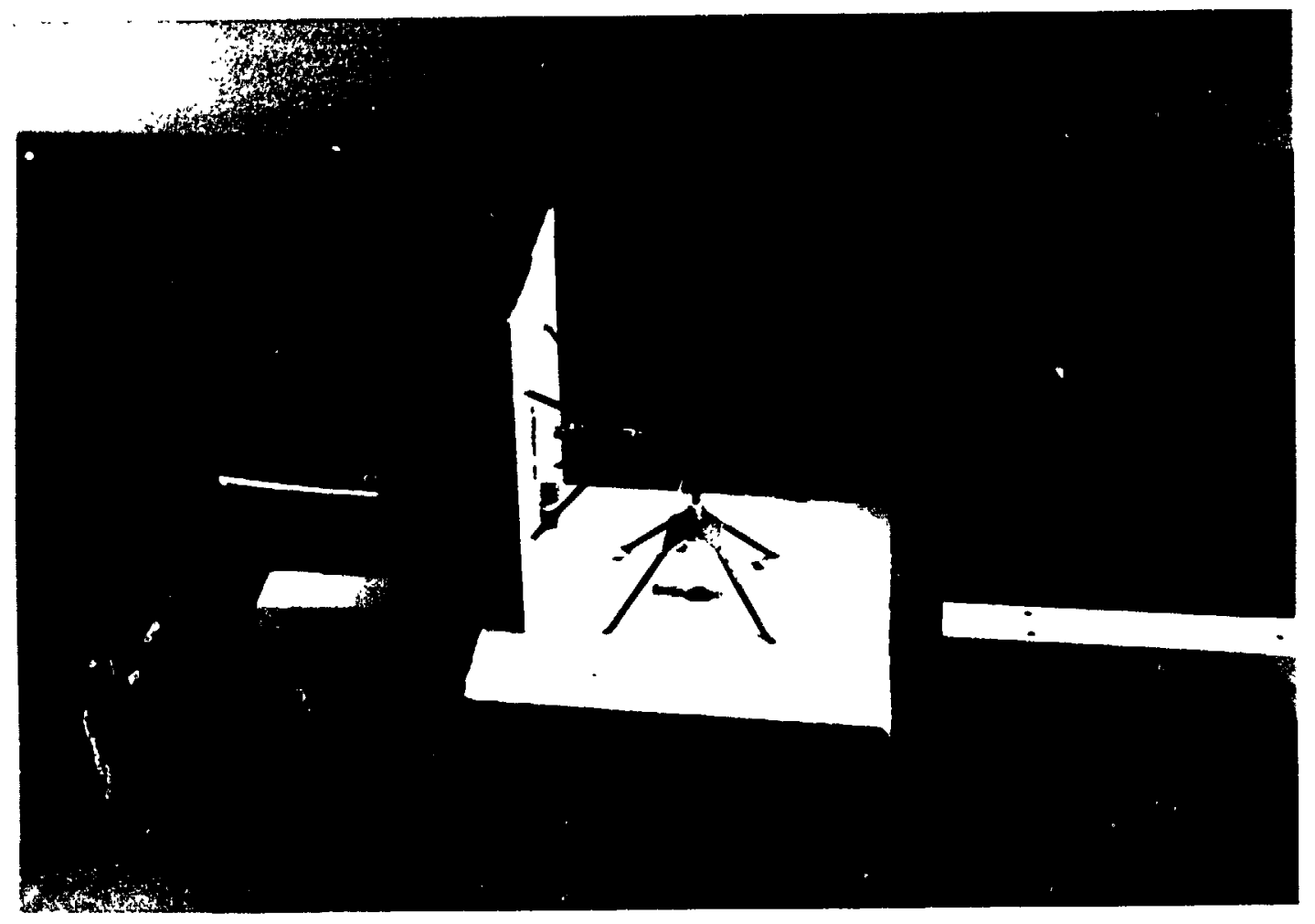

Plate 6 Holding frame for polarization diversity antenna 


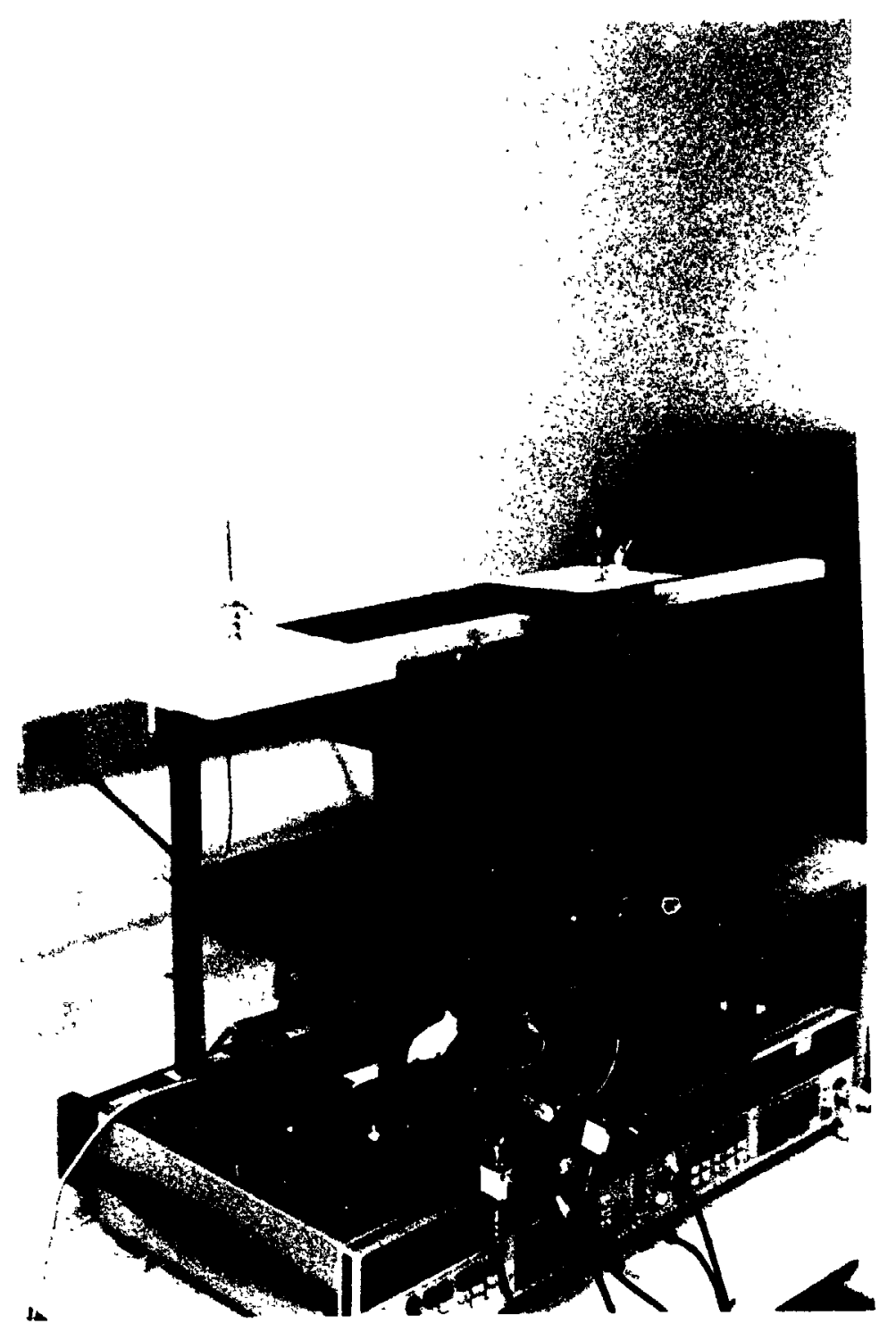

Plate 7 Guiding frame used to take measurement 


\section{Bibliography}

[1] A. Motley, "Advanced Cordless Telecommunications Service," I.E.E.E. Journal on Selected Areas in Communications, vol. SAC-5, no. 5, pp. 774-782, June 1987.

[2] D.C.Cox, "Universal Portable Radio Communications," I.E.E.E. Transactions on vehicular technology, vol. VT-34, no. 3, pp. 117-121, August 1985.

[3] M. Kavehrad and G. Bodeep, "Design and Experimental Results for a DirectSequence Spread-Spectrum Radio Usir̈g Differential Phase-Shift Keying Modulation for Indoor, Wireless Communications," I.E.E.E. Journal on Selected Areas in Communications, vol. SAC-5, no. 5, pp. 815-823, June 1987.

[4] T. Rappaport and C. McGillem, "UHF Fading in Factories," I.E.E.E. Journal on Selected Areas in Communications, vol. 7, no. 1, pp. 40-48, January 1989.

[5] T. Rappaport and C. McGillem, "Charaterizing the UHF Factory Radio Channel," Electronics Letters, vol. 23, July 1987.

[6] T. Rappaport, "Characterization of UHF Multipath Radio Channels in Factory Buildings," I.E.E.E. Transactions on Antennas and Propagation, vol. 37, no. 8, pp. 1058-1069, August 1989.

[7] A. Acampora and J. Winters, "System Applications for Wireless Indoor Communications," I.E.E.E. Communications Magazine, vol. 25, no. 8, pp. 11-20, August 1987.

18.) S. Stein, "Fading Channel Issues in System Engineering," I.E.E.E. Journal on Selected Areas in Communications, vol. SAC-5, no. 2, pp. 68-89, February 1987.

[9] J. Proakis Digital Communications, McGraw-Hill, New York, 1983.

[10] A. Saleh and R. Valenzuela, "A Statistical Model for Indoor Multipath Propagation," I.E.E.E. Journal on Selected Areas in Communications, vol. SAC-5, no. 2 , pp. 128-137, February 1987. 
[11] D. Devasirvatham, "Time Delay Spread and Signal Level Measurements of 850 MHz Radio Waves in Building Environments," I.E.E.E. Transactions on Ansennas and Propagation, vol. AP-34, no. 11, pp. 1300-1305, November 1986.

[12] W.C.Jakes, "chapter 5," Microwave Mobile Communications, Wiley, New York, 1974.

[13] D. Cox, R. Murray, H. Amold, A. Norris, and M. Wazowicz, "Cross-Polarization Coupling Measured for $800 \mathrm{MHz}$ Radio Transmission In and Around Houses and Large Buildings," I.E.E.E. Transactions on Antennas and Propagation, vol. AP34, no. 1, pp. 83-87, January 1986.

[14] W. Lee, "chapter 10," Mobile Communications Engineering, McGraw-Hill, New York, 1982.

[15] S. Alexander, "Characterising Buildings for Propagation at $900 \mathrm{MHz}$," Electronics Letters, vol. 19, no. 20, p. 860, 29 September 1983.

[16] S. Alexander, "Radio Propagation within Buildings at $900 \mathrm{MHz}$," Electronics Letters, vol. 18, no. 21, pp. 913-914, October 1982.

[17] R. Bultitude, "Measurement,Characterization and Modeling of indoor 800/900 $\mathrm{MHz}$ Channels for Digital Communications," I.E.E.E. Communications Magazine, vol. 25, no. 6, pp. 5-12, June 1987.

[18] A. Acampora and J. Winters, "A Wireless Network for Wide-Band Indoor Communications," I.E.E.E. Journal on Selected Areas in Communications, vol. SAC-5, no. 5, pp. 796-805, June 1987.

[19] A. Saleh and L. Cimini, "Indoor Radio Communications Using Time-Division Multiple Access with Cyclical Slow Frequency Hopping and Coding," I.E.E.E. Journal on Selected Areas in Communications, vol. 7, no. 1, pp. 59-70, January 1989.

[20] J. Winters and Y. Yeh, "On the Pelformance of Wideband Digital Radio Transmission Within Buildings Using Diversity," in GLOBECOM '85, pp. 991996, December 1985. 
121] E. Chien, D. Goodman, and J. R. Sr, “A System's Approach to Indoor Wireless Communications," in GLOBECOM' 87, pp. 1931-1935, 1987.

[22] H. Hoffman and D. Cox, "Attenuation of $900 \mathrm{MHz}$ Radio Waves Propagating into a Metal Building," I.E.E.E. Transactions on Antennas and Propagation, vol. AP30, no. 4, pp. 808-811, July 1982.

[23] D. Cox, R. Murray, and A. Norris, "Measurements of $800-\mathrm{MHz}$ Radio Transmission Into Buildings With Metallic Walls," The Bell System Technical Journal, vol. 62, no. 9, pp. 2695-2717, November 1983.

[24] S. Alexander and G. Pugliese, "Cordless communication within buildings: reuslts of measurements at $900 \mathrm{MHz}$ and $60 \mathrm{GHz}$," British Telecom Technology Journal, vol. 1, no. 1, pp. 99-105, July 1983.

[25] A. Motley and S. Alexander, "Diversity Advantage for Cordless Telephones," Electronics Letters, vol. 19, no. 14, pp. 530-533, July 1983.

[26] S. Patsiokas, B. Johnson, and 1. Dailing, "Propagation of Radio Signals Inside Buildings at 150,450 and $850 \mathrm{MHz}, "$ in Procedings Vehicular Technology Conference, pp. 66-7i, May 1986.

[27] D. Devasirvatham, "A Comparison of Time Delay Spread and Signal Level Measurements Within Two Dissimilar Office Buildings," I.E.E.E. Transactions on Antennas and Propagation, vol. AP-35, no. 3, pp. 319-324, March 1987.

[28] W. Lee and Y. Yeh, "Polarization Diversity for Mobile Radio," I.E.E.E. Transactions on Communications, vol. COM-20, no. 5, pp. 912-923, October 1972.

[29] A. Saleh, A. Kustako, and R. Roman, "Distributed Antennas for Indoor Radio Communications," I.E.E.E. Transactions on Communications, vol. COM-35, no. 12, pp. 12:5-1251, December 1987.

[30] G. Reed and C. Stevenson, "Indoor Radio Propagation Measurements," not published, A.T. and T. Bell Lab Whippany, NJ.

[3!] D. Cnx, "Delay Do pler Characieristics of multipath propagation at $910 \mathrm{MHz}$ in a suburban mobile radio environment," I.E.E.E. Transactions on Antennas and 
Propagation, vol. AP-20, no. 5, pp. 625-635, September 1972.

[32] R. Swain, "Cordless telecommunications in the UK," British Telecom Technology Journal, vol. 3, no. 2, pp. 32-38, April 1985.

[33] D. Devasirvatham, "Time Delay Spread Measurements of Wideband Radio Signals Within Building," Electronics Letters, vol. 20, no. 23, pp. 950-951, November 1984.

[34] W. Jakes Microwave Mobile Communications, Wiley, New York, 1974. 


\section{Appendix A Program Listings}

The program listings shown here are the program used for data acquisition and the main calculation programs. The short prcgrams are listed in there entirety but the longer ones are not: only the appropriate segments are listed. The algorithms for these programs were explained in chapter 3 and 4.

The programs listed in this appendix are the following:

1. ADSAMP.BAS;

2. DATASCALE;

3. DATAVG;

4. DATACDF; and

5. LOSS.

\section{A.1 ADSAMP.BAS}

This program is the data acquisition control program explained in section 3.9.2. It is written in GW-BASIC.

10' ADSAMP.BAS

$20^{\prime}$ DATA ACQUISITION CONTROL PROGRAM

30 ' using mode $0,1,17,20,8$ and 9

$40^{\circ}$ Mode 0,17,1,20,8 and mode 9 are used. 2-03-89

50 .

60 COL.OR 11,0 'screen setting

70 DEF SEG $=\& H 8000$ ' memory address to load machine language driver 80 DIM DIO\%(4),DT\%(20000)

90 PROGRAM INITIALIZATION

1(K) BLOAD "DASH16.BIN",0 ' load driver in memory at offset 0 from DEI: SEG 
110 RATE $1 \%=20:$ RATE2 $\%=100$ ' timer rate settings

120 DMA\%=\&H2000: DAT\%=\&H2000 ' DMA and DATA address

130 INPUT "NAME OF FILE TO STORE DATA:",FLNM\$

140 INPUT "ANTENNA SPACING:1, $2,3,4,5$ OR 0 IF SPACING NOT

IMPORTANT: ",SP\%

150 INPUT "TOTAL NO OF CONVERSIONS (default=60 000):",INCON-

VER\% 'no entry $=60000$

160 INPUT "SAMPLING RATE? (default=500 Hz) ",INRATE\%

170 IF INRATE $\% \propto 0$ THEN RATE1 $\%=10000 /$ NRATE $\%$ ' for ' 'ifferent

rates

180 IF INCONVER\% $<=20000$ THEN FLG $=1^{\prime}$ an entry $<20000$ is a test run

190 IF INCONVER\% $=0$ THEN FLG $=2$

200 IF INCONVER\% $>20000$ THEN GOTO 150

210 ON FLG GOTO 220,270

205 ' case for tesi run

220 CONVER $\%=I N C O N V E R \%$

230 TOTAL $=$ CONVER\%

240 TRNS\%=INCONVER\%

$250 \mathrm{CNT}=0:$ TRNSCNT $=0$

260 GOTO 320

$265^{\prime}$ case for data run :

270 CONVER $\%=30000$ 'default samples: $2 \times 30000$

$280 \mathrm{CNT}=2$ :TRNSCNT $=4$ 'CNT:sampling run counter,

290 TOTAL $=C N T *$ CONVER\% 'TRNSCNT:data transfer counter

300 TRNS\% $\%=15000$ ' default transfer: $4 \times 15000$

$310^{\prime}$ - MODE 0 Board Initialization-

320 DIO\% $(0)=768^{\prime}$ H/O address of board

$330 \mathrm{DIO} \%(1)=2: \mathrm{DIO} \%(2)=1$ 'interrupt level 
340 DASH16 $=0$ 'call offset from DEF SEG

350 FLAG\% $=0$ 'error variable

$360 \mathrm{MD} \%=0$ 'mode 0 - initialize

370 CALL DASH16 (MD\%, DIO\%(0), FLAG\%)

380 IF RLAG\% $\% 0$ THEN PRINT "INSTALLATION ERROR":STOP 'Halt

on error

$390^{\prime}-$ MODE $17-$ Sampling rate-

400 ,

410 DIO\%(0)=RATE1\%' timer rate $=1 \mathrm{MHz} /($ Rate $1 \times$ Rate2 $)$

420 DIO\%(1) $=$ RATE2\% 'product $=2000=500 \mathrm{HZ}$

$430 \mathrm{MD} \%=17$ 'timer set mode

440 CALL DASH16 (MD\%, DIO\%(0),FLAG\%)

450 IF FLAG\%<>0 THEN PRINT "Error in setting timer. Error \#

";FLAG\%:STOP

$460^{\circ}$-mode 1 block scan limits-

470 LL\% $=0:$ UL\%=1

480 DIO\% $(0)=$ LL\% 'lower limit

490 DIO\%(1) = UL\% 'upper limit

$500 \mathrm{MD} \%=1$

510 CALL DASH16 (MD\%, DIO\%(0),Fl AG\%)

520 IF FLAG\% $>0$ THEN PRINT"Error in setting scan limits \# ";FLAG\%

: STOP

530 - MODE 20 block conversions-

540 DMA\%=DMA\%+\&H1000 : PRINT DMA\% ' increment address of memory location to store conversions

550 DIO\% $(0)=$ CONVER\% 'No of conversions $=30 \mathrm{~K}$

560 DIO\%(1) $=$ DMA\% 'Memory segment to dump data

$570 \mathrm{DIO} \%(2)=1$

$580 \mathrm{DI} \% \%(3)=0$ 
$590 \mathrm{MD} \%=20$ 'mode 20 - block scan on interrupt

600 BEEP

610 CALL DASH16 (MD\%, DIO\%(0), FLAG\%) 'set it going

620 IF FLAG\% $>0$ THEN PRINT"Error in block scan setup =

";FLAG\%:STOP

630 - MODE 8 waiting for the end of conversions

$640 \mathrm{MD} \%=8$

650 CALL DASH16 (MD\%, DIO\%(0), FLAG\%) 'montoring block scan

660 IF DI0\%(1)=1 THEN PRINT DIO\%(1)

670 IF DIO\%(1) $=0$ THEN GOTO 690

680 GOTO 640

$690 \mathrm{CNT}=\mathrm{CNT}-1$ ' block counter decreased

700 IF $\mathrm{CNT}<=0$ GOTO 730

710 GOTO 400

720 '-Opening of transfer file-

730 BEEP

740 PIP\$="VHPBASI"+FLNM\$ ' name of storage file

750 OPEN "O",\#1,PIPS ' establishing path to file

760 PRINT \#1,FLNM\$ ' printing file info

770 WRITE\#1,SP\%,TOTAL

$780^{\prime}-$ MODE 9 data from memory to array

$790 \mathrm{MD} \%=9$

800 DAT\%=DAT\%+\&H1000 ' address of mem location to get data

810 DIO\%(0)=TRNS\% ' no of data to tra..sfer: $15 \mathrm{~K}$ default

820 DIO\%(1) $=$ DAT $\%$ ' address to get data

$830 \mathrm{DIO} \%(2)=0$ ' offset from address

840 PRINT DIO\%(1)

850 DIO\%(3) = VARPTR(DT\%(0)) 'indicates tzeginning of array in program

860 DIO\%(4) $=0$ 
870 CALL DASH16 (MD\%, DIO\%(0), FLAG\%)

$880 \mathrm{IF}$ FLAG\%< $>0$ THEN PRINT"Error data transf ${ }^{\circ}=$ = "FLAG\%:STOP

'any errors?

$885^{\prime}$ 'writing array to file on disk, 20 values per loop

890 FOR I $=0$ TO TRNS $\%-1$ STEP 20

900 WRITE\#1,DT\%(I),DT\%(I+1),DT\%(I+2),DT\%(I+3),DT\%(I+4),

DT\%(I+5),DT\%(I+6), DT\%(I+7),DT\%(I+8),DT\%(I+9)

910 WRITE\#1,DT\%(I+10),DT\%(I+11),DT\%(I+12),DT\%(I+13),DT\%(I+14),

DT\%(I+15),DT\%(I+16),DT\%(I+17),DT\%(I+18),DT\%(I+19)

920 NEXT 1

930 IF TRNS\%=CONVER\% GOTO 1120 'test to see if any more data

940 TRNSCNT $=$ TRNSCNT-1 ' decrease transfer counter

$950 \mathrm{IF}$ TRNSCNT $<=0$ GOTO 1120

$960 \mathrm{MD} \%=9$ 'starting 2 nd half of $30 \mathrm{~K}$ block

$970 \mathrm{DIO} \%(0)=$ TRNS\%

980 DIO $\%(1)=$ DAT $\%$

990 DIO\%(2) = TRNS\% ' offset from memory address: 2 nd half

1000 PRINT DIO\%(1)

1010 DIO\%(3) = VARPTR(DT\%(0))

$1020 \mathrm{DIO} \%(4)=0$

1030 CALL DASH16 (MD\%, DIO\%(0), FLAG\%)

1040 IF FLAG\%<>0 THEN PRINT"Error data transfer = ";FLAG\%:STOP

'any errors?

1045 ' writing array to file

1050 FOR I $=0$ TO TRNS\%-1 STEP 20

1060 WRITE\#1,DT\%(I),DT\%(I+1),DT\%(I+2),DT\%(I+3),DT\%(I+4),

DT\%(I+5),DT\%(I+6),DT\%(I+7),DT\%(I+8),DT ।

1070 WRITE\#1,DT\%(I+10),DT\%(I+11),DT\%(1 JT\%(I+13),DT\%(I+14),

DT\%(I+15),DT\%(I+16),DT\%(I+17),DT\%(I+18),DT\%(I+19) 
1080 NEXT I

1090 TRNSCNT $=$ TRNSCNT-1 ' decrease transfer counter

1100 IF TRNSCNT $<=0$ GOTO 1120

1110 GOTO 790

1120 CLOSE\# 1

1130 STOP

1140 END

\section{A.2 DATASCALE}

This program does the scaling of data file: it transforms the raw analog-to-digital converter values to power values in $\mathrm{dBm}$.

10 ! SCALING PROGRAM

20 !

30 ! DATASCALE1

40 !

50 ! THIS PROGRAM TAKES THE DATA FROM A FILE, RUNS IT THROUGH

60 ! A SCALING ROUTINE TO CONVERT THE A/D READINGS TO DBM.

70 ! IT THEN LOADS "DATACDF"

80 ! THE VALUES IN P1(*),P2(*) ARE PASSED ALONG THROUGH A COM BLOCK.

$90 !$ - LOAD BIN "IO" BEFORE RUNNING THE PROG

100 WAIT 1

110 OPTION BASE 1

120 INTEGER Min1,Min2,Delmin

The COM block is used to pass information between the various programs being loaded 
130 COM REAL P1(30000), P2(30000), Total, Name\$[12], Dir\$(115)[80], Vol\$(3)[12], INTEGER Sp, Prog, Filno, Rtype, Maxfil, V

140 ALLOCATE INTEGER D1(30000)

150 DIM Filnm\$[21]

160 GOSUB Clears

170 Prog=1

$180 \mathrm{Ms} \$=" "$

190 IF Rtype $<>0$ THEN GOTO S2

Allows for batch mode if program is to run unattended

$200 \mathrm{~S}$ :INPUT "do you want to run in batch mode: 1-yes, 2-no",Ba

210 INPUT "enter initial value and max value of FILNO", Startfil, Maxfil

This loop allows to read data from more than one directory 220 FOR $\mathrm{K}=1$ TO 3

230 LINPUT "ENTER VOLUME WHERE THE DATA FILES ARE:", Vol\$(K)

240 PRINT Vol\$(K)

250 NEXT K

$260 \mathrm{~V}=1$ !

270 Filno=St:rtfil

280 ON Ba GUTO S2,S3

290 S2:IF Rtype=2 THEN GOTO S3 ! INTERAC MODE

300 S21:IF Filno $>=$ Maxfil +1 THEN

$310 \mathrm{~V}=\mathrm{V}+1$

320 Filno $=1$

330 ON V GOTO S21,S21,Fin

340 Fin:BEEP

350 GOSUB Clears

360 PRINT USING "5/"

370 PRINT " THE END " 
380 PRINT "all files are done "

390 Filno=1

400 STOP

410 ELSE

420 Rtype $=1$ ! batch mode

430 IF Filno=1 THEN GOSUB Catdump ! Catdump write the directory to the vector Dir\$

440 IF LEN(TRIM\$(Dir\$(Filno)[1,12]))<7 THEN ! non data files have smaller file name: they are not selected

450 Filno=Filno +1

460 GOTO S21

470 ELSE

480 Name $\$=\operatorname{TRIM} \$$ (Dir\$(Filno) $[1,12]) !$ data file names is read into Name\$ 490 Filnm $\$=$ Name $\$$ Vol $\$(V)$

500 PRINT Name\$

510 GOTO S5

520 END IF

530 END IF

The program section from line 540 to 770 deals with non batch mode and no scaling operations

780 S5:In $\$=$ Name $\$[1,1]$ ! first letter of file name indicates the diversity type 790 ASSIGN @F TO Filnm\$! path to file

800 ENTER @F;N\$,Sp,Total

810 REDIM D1(Total),P1(Total),P2(Total)

820 ENTER @F;Tim,D1(*) ! entering channel 1 data

830 IF In $\$=" F "$ THEN

840 Scale\$=In\$\&"SCALE"\&VAL\$(Sp)! loading the proper scaling file 850 ELSE

860 Scale\$="SSCALE" ! loading the proper scaling file 
870 END IF

880 ALLOCATE REAL Ad(0:4095)

890 ASSIGN@S TO Scale\$ ! path to scaling file

900 ENTER @S;Ad(*) ! entering scaling vector for chan 1

910 ALLOCATE INTEGER D2(30000)

920 REDIM D2(Total)

930 ENTER @F;D2(*) ! entering channel 2 data

Doing manipulations needed to do the correction on channel 1's data $940 \mathrm{Min} 1=\operatorname{MIN}\left(\mathrm{D} 1\left(^{*}\right)\right)$

$950 \mathrm{Min} 2=\mathrm{MIN}\left(\mathrm{D} 2\left(^{*}\right)\right)$

960 OUTPUT 26;N\$,"MIN1";Min1,"MIN2"; Min2

970 IF Min $1<$ Min2 THEN GOTO S6

980 Delmin=Min1-Min2

990 MAT D1= D1-(Delmin)

1000 !

1010 S6:DISP "S6: scaling D1"

1020 FOR I=1 TO Total STEP 15

1030 Pl(I)=Ad(D1(I)) ! Scaling channel 1

$1040 \mathrm{Pl}(\mathrm{I}+1)=\operatorname{Ad}(\mathrm{D} 1(\mathrm{I}+1))$

$1050 \mathrm{Pl}(\mathrm{I}+2)=\mathrm{Ad}(\mathrm{D} 1(\mathrm{I}+2))$

$1060 \mathrm{P} 1(\mathrm{I}+3)=\operatorname{Ad}(\mathrm{D} 1(\mathrm{I}+3))$

$1070 \mathrm{Pl}(\mathrm{I}+4)=\mathrm{Ad}(\mathrm{D} 1(\mathrm{I}+4))$

$1080 \mathrm{P} !(\mathrm{I}+5)=\mathrm{Ad}(\mathrm{D} 1(\mathrm{I}+5))$

$\mathrm{i} 090 \mathrm{Pl}(\mathrm{I}+6)=\operatorname{Ad}(\mathrm{D} 1(\mathrm{I}+6))$

$1100 \mathrm{Pl}(\mathrm{I}+7)=\mathrm{Ad}(\mathrm{D} 1(\mathrm{I}+7))$

$1110 \mathrm{P} 1(\mathrm{I}+8)=\mathrm{Ad}(\mathrm{D} 1(\mathrm{l}+8))$

$1120 \mathrm{P} 1(\mathrm{I}+9)=\mathrm{Ad}(\mathrm{D} 1(\mathrm{I}+9))$

$1130 \mathrm{Pl}(1+10)=\operatorname{Ad}(\mathrm{D} 1(\mathrm{I}+10))$

$1140 \mathrm{Pl}(1+11)=\operatorname{Ad}(\mathrm{D} 1(1+11))$ 
$1150 \mathrm{Pl}(\mathrm{I}+12)=\mathrm{Ad}(\mathrm{D} 1(\mathrm{I}+12))$

$1160 \mathrm{P} 1(\mathrm{I}+13)=\operatorname{Ad}(\mathrm{D} 1(\mathrm{I}+13))$

$1170 \mathrm{P} 1(\mathrm{I}+14)=\operatorname{Ad}(\mathrm{D} 1(\mathrm{I}+14))$

1180 NEXT I

1190 !

1200! ASSIGN @F TO *

1210 !

1220 ENTER @S; $\operatorname{Ad}\left({ }^{*}\right) !$ entering scalino vector for chan 2

1230! ASSIGN @S TO *

1240 !

1250 DISP "S7:scaling D2"

1260 S7:FOR I=1 TO Total STEP 15

$1270 \mathrm{P} 2(\mathrm{I})=\operatorname{Ad}(\mathrm{D} 2(\mathrm{I}))$ ! SCALING P2

$1280 \mathrm{P} 2(\mathrm{I}+1)=\operatorname{Ad}(\mathrm{D} 2(\mathrm{I}+1))$

$1290 \mathrm{P} 2(\mathrm{I}+2)=\mathrm{Ad}(\mathrm{D} 2(\mathrm{I}+2))$

$1300 \mathrm{P} 2(\mathrm{I}+3)=\mathrm{Ad}(\mathrm{D} 2(\mathrm{I}+3))$

$1310 \mathrm{P} 2(\mathrm{I}+4)=\mathrm{Ad}(\mathrm{D} 2(\mathrm{I}+4))$

$1320 \mathrm{P} 2(\mathrm{I}+5)=\operatorname{Ad}(\mathrm{D} 2(\mathrm{I}+5))$

$1330 \mathrm{P} 2(\mathrm{I}+6)=\mathrm{Ad}(\mathrm{D} 2(\mathrm{I}+6))$

$1340 \mathrm{P} 2(\mathrm{I}+7)=\operatorname{Ad}(\mathrm{D} 2(\mathrm{I}+7))$

$1350 \mathrm{P} 2(\mathrm{I}+8)=\operatorname{Ad}(\mathrm{D} 2(\mathrm{I}+8))$

$1360 \mathrm{P} 2(\mathrm{I}+9)=\mathrm{A} A(\mathrm{D} 2(\mathrm{I}+9))$

$1370 \mathrm{P} 2(\mathrm{I}+10)=\mathrm{Ad}(\mathrm{D} 2(\mathrm{I}+10))$

$1380 \mathrm{P} 2(\mathrm{I}+11)=\mathrm{Ad}(\mathrm{D} 2(\mathrm{I}+11))$

$1390 \mathrm{P} 2(\mathrm{I}+12)=\mathrm{Ad}(\mathrm{D} 2(\mathrm{I}+12))$

$1400 \mathrm{P} 2(\mathrm{I}+13)=\mathrm{Ad}(\mathrm{D} 2(\mathrm{I}+13))$

1410 P?(I+14)-Ad(D2(I-14))

1420 NEXT I

1430 DEALLOCATE Ad(*),D2(*) 
$1440 !$

1450 ON Rtype GOTO Cd,S8 ! for batch mode Rtype =1

1460 S8:INPUT "1-work on CDF, 2-plot on the screen",En

1470 ON En GOTO Cd,Scm

1480 Cd:PR.INT " LOADING DATACDF"

1490 LOAN "DATACDF"

1500 Scm:PRINT

1510 PRINT " LOADING DATASCREEN"

1520 PRINT

1530 LOAD "DATASCREEN"

$1540 !$

$1550 !$

1560 Catdump:CAT Vol\$(V) TO Dir $\$(*)$;COUNT T,NO HEADER ! ,8

1570 REDIM Dir\$(T)

1580 RETURN

1590 Clears:OUTPUT KBD USING "\#,B";255,75

1600 RETURN

1610 END

\section{A.3 DATAVG}

This program calculates the average power of channel 1 and channel 2 and write the two values in a results file. To calculate the arithmetic average the scaling is done using the milliwatt scaling files.

10 ! AVERAGE CALCULATION PKOGRAM

20 !

30! DATAVG

40 !

50 ! THIS PROGRAM TAKES THE DATA FROM A FILE, RUNS IT THROUGH 
60 ! A SCALING ROUTINE TO CONVERT THE AND READINGS TO $\mathrm{mW}$.

70 ! IT THEN CALCULATES THE ARITHMETIC AVERAGE OF BOTH SIGNAL.

80 ! THESE AVERAGE ARE STORED IN A RESULT FILE CREATED IN VHPBASTRES1

90 OPTION BASE 1

100 COM REAL P1(30000),P2(30000),Total,Name\$i 12], Dir\$(20)[80],

INTEGER Sp, Prog, Filno, Rtype, Maxfil

110 INTEGER Disk

120 REAL Mw(0:4095),Atten(10)

130 DIM Rname\$[21],Filnm\$[21]

140 Prog $=5$

150 Vol $\$=": C S 80,1500,9 "$

160 GOSUB Catdump

170 PRINT Dir\$(*)

The program section not shown was dealing with entering the attenuation for each channe! and writing to the table of content file.

320 ALLOCATE INTEGER D1(30000)

330 ASSIGN @F TO Filnm\$\&Vol\$

340 ENTER @F;N\$,Sp,Total

350 REDIM D1(Total),P1(Total),P2(Total)

360 ENTER @F;Tim,Dl(*)

370 PRINT " ",K,N\$

380 IF In $\$=" F "$ THEN

390 Scale $\$=$ In \&\&"SCALE"\&VAL $(S p) \& " W " !$ the W indicates it is a $\mathrm{mW}$ file

400 ELSE

410 Scale\$="SSCALEW"!MEM,0,1" 
420 END IF

430 ASSIGN@S TO Scale\$

440 ENTER @S;Mw(*)! TABLE FOR P1

450! !

460 S3: DISP " scaling D1"

470 FOR I=1 TO Total STEP 10

$480 \mathrm{Pl}(\mathrm{I})=\mathrm{Mw}(\mathrm{D} 1(\mathrm{I}))$ ! SCALING P1

$490 \mathrm{Pl}(\mathrm{I}+1)=\mathrm{Mw}(\mathrm{D} 1(\mathrm{I}+1)$ !

$500 \mathrm{Pl}(\mathrm{I}+2)=\mathrm{Mw}(\mathrm{D} 1(\mathrm{I}+2))$

$510 \mathrm{P} 1(\mathrm{I}+3)=\mathrm{Mw}(\mathrm{D} 1(\mathrm{I}+3))$

$520 \mathrm{Pl}(\mathrm{I}+4)=\mathrm{Mw}(\mathrm{D} 1(\mathrm{I}+4))$

$530 \mathrm{Pl}(\mathrm{I}+5)=\mathrm{Mw}(\mathrm{Dl}(\mathrm{I}+5))$

$540 \mathrm{Pl}(\mathrm{I}+6)=\mathrm{Mw}(\mathrm{D} 1(\mathrm{I}+6))$

$550 \mathrm{Pl}(\mathrm{I}+7)=\mathrm{Mw}(\mathrm{D} 1(\mathrm{I}+7))$

$560 \mathrm{Pl}(\mathrm{I}+8)=\mathrm{Mw}(\mathrm{D} 1(\mathrm{I}+8))$

$570 \mathrm{Pl}(\mathrm{I}+9)=\mathrm{Mw}(\mathrm{Dl} 1(\mathrm{I}+9))$

580 NEXT I

590 !

600 DEALLOCATE D1(*)

610 ALLOCATE INTEGER D2(30000)

620 REDIM D2(Total)

630 ENTER @F;D2(*)

640 ASSIGN@F TO *

$650 !$

660 ENTER @S;Mw(*)! TABLE FOR P2

670 ASSIGN@S TO *

$680 !$

690 DISP " scaling D2"

700 S4: FOR I=1 TO Total STEP 10 
$710 \mathrm{P} 2(\mathrm{I})=\mathrm{Mw}(\mathrm{D} 2(\mathrm{I})) !$ SCALING $\mathrm{F} 2$

$720 \mathrm{P} 2(\mathrm{I}+1)=\mathrm{Mw}(\mathrm{D} 2(\mathrm{I}+1))$

$730 \mathrm{P} 2(\mathrm{I}+2)=\mathrm{Mw}(\mathrm{D} 2(\mathrm{I}+2))$

$740 \mathrm{P} 2(\mathrm{I}+3)=\mathrm{Mw}(\mathrm{D} 2(\mathrm{I}+3))$

$750 \mathrm{P} 2(\mathrm{I}+4)=\mathrm{Mw}(\mathrm{D} 2(\mathrm{I}+4))$

$760 \mathrm{P} 2(\mathrm{I}+5)=\mathrm{Mw}(\mathrm{D} 2(\mathrm{I}+5))$

$770 \mathrm{P} 2(\mathrm{I}+6)=\mathrm{Mw}(\mathrm{D} 2(\mathrm{I}+6))$

$780 \mathrm{P} 2(\mathrm{I}+7)=\mathrm{Mw}(\mathrm{D} 2(\mathrm{I}+7))$

$790 \mathrm{P} 2(\mathrm{I}+8)=\mathrm{Mw}(\mathrm{D} 2(\mathrm{I}+8))$

$800 \mathrm{P} 2(\mathrm{I}+9)=\mathrm{Mw}(\mathrm{D} 2(\mathrm{I}+9))$

810 NEXT I

820 DEALLOCATE D2(*)

830 ! calculating the average in $\mathrm{mW}$

840 DISP "CALCULATING AVERAGE OF ",N\$

850 Ave $1=S U M(P 1)$

860 Ave2=SUM(P2)

870 Ave1=Ave1/Total

880 Ave2=Ave2/Total

Converting the $m W$ values into $\mathrm{dBm}$ values

890 Ave $1=10 *$ LGT(Ave 1)

900 Ave2=10*LGT(Ave2)

910 OUTPUT $26 ; \mathrm{N \$}$

920 OUTPUT 26;Ave1," ",Ave2

930 OUTPUT 26;" "

Creating the result files: the file name is based on the data file name 940 IF POS(Dir\$(K)[1,9],"_")=4 THEN

950 Rname $\$=\operatorname{Dir} \$(K)[1,3] \& "$ R" \&":CS80,1500,9"

960 ELSE 


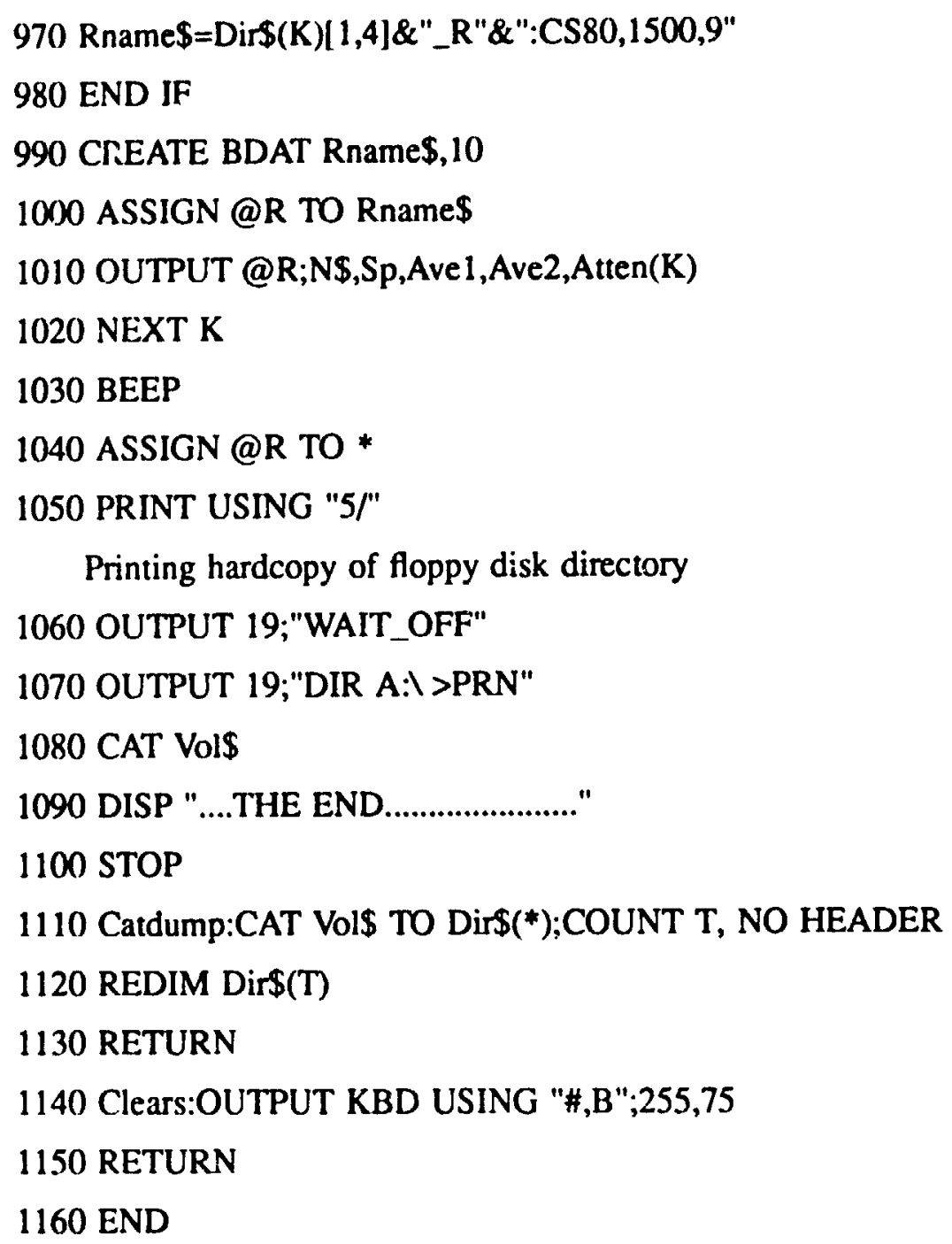

\section{A.4 DATACDF}

This program is the main calculation program. It took the scaled data from the DATASCALE program and the average power value stored in the result file and calculated the histogram boundaries, the histogram itself and the climulative distribution function. The distribution was stored in the result file for future access. 
The program was designed to allow interactive or batch operations. The interactive mode allowed many functions to be accessed including plotting on the monitor and on an hard copy plotter connected to the PC. These parts of the program will not be listed.

10 ! DATACDF: THE STATISTICS PROGRAM

$20 !$

30 ! THIS PROGRAM TAR̈ES THE DATA FROM A COM BLOCK AND CALCULATES PDF, CDF 40 ! FOR P1,P2 AND MAX (P1,P2) .OPTION TO PLOT ON SCREEN AND PLOTTER.

50! OPTION 'TO CREATE RESULTS FILE AND STORE CDF VALUES.

60 !

70 OPTION BASE 1

COM block used to pass data from the DATASCALE program.

80 COM P1(30000), P2(30000), Total, Name\$[12], Dir\$(115)[80], Vol\$(3)[12], INTEGER Sp, Prog, Filno, Rtype, Maxfil,V

Vectors used for boundary, histogram and cdf counters

90 REAL X1(41), X2(41), Pd1(42),Pd2(42),Cd!(42),Cd2(42), Pd3(42), Cd3(42)

100 REAL Dra(42),Cd12(42),Cdjt(42),Pdjt(42),Cd(42), Pd(42)

110 DIM Ylab\$(10)[3],Ylab1\$[25],Xlab\$(21)[3], Spac\$(11)|6], Title1\$150]

120 DIM Div\$(0:11)[8]

130 REDIM P1(Total),P2(Total)

140 GOSUB Clears

These are the function allowing interactive use of the program.

150 ON KEY 5 GOSUB Cdf12 ! F1 FOR CDF OF P1,P2

160 ON KEY 6 GOSUB Getresf! F2 TO GET A RESFILE

170 ON KEY 7 GOSUB Jnt12！F3 FOR JOINT CDF OF P1,P2

180 ON KEY 8 GOTO More ! F4 END PRUGR

190 ON KEY 0 GOSUB Resfile ! Sh-F1 TO STORE. INFO IN RESULT FIL.E

200 ON KEY 2 GOSUB Med! Sh-F3 MEDIAN OF P1,P2 
210 ON KEY 1 GOSUB Hpplo! Sh-F2 TO PLOT ANY CDF

220 ON KEY 3 GOSUB Screen! Sh-F3 TO PLOT ON SCREEN

230 !

240 Bdy=31! FROM AVE-30 TO AVE $+10^{\circ}$

250 GOSUB Pdfbdry ! TO GET X LABEL VALUES FOR PLOT

260 C2:IF Rtype=1 THEN GOTO Batchrun ! batch mode, no interaction

$270 \mathrm{Ch}: G O S U B$ Menu ! menu for interactive mode

$280 \mathrm{Nex}=1$

290 Wait:IF Nex=2 THEN GOTO $\mathrm{Ch}$

300 GOTO Wait

310 More:GRAPHICS OFF

320 INPUT "TYPE 1-STOP, 2-SCALE NEW FILE, 3-CARRY ON", En

330 ON En GOTO Fin, Sc, Ch

340 Fin:PRINT "...THE END ....."

350 STOP

$360 \mathrm{Sc}: D I S P$ "LOADING DATASCALE"

370 rrog $=3$

380 LOAD "DATASCALE"

390 !

400 !

410 Batchrun:GOSUB Cdf 12 ! doing individual channels cdf

420 GOSUB Jnt 12 ! doing joint cdf

440 Filno=Filno +1

450 LOAD "DATASCALE1" ! when cdf are done for this file, return to scaling

program to do next file

460 !

470 !

480 Cdf 12: GOSUB Getresf ! to get data from result file

490 GOSLB Pdfbdry ! calculating the histogram boundaries 
500 GOSUB Pdf ! doing histogram (or pdf)

510 MAT Pd= Pd1

520 GOSUB Cdf ! doing cdf

530 MAT $\mathrm{Cd} 1=\mathrm{Cd}$

540 MAT $\mathrm{Pd}=\mathrm{Pd} 2$

550 GOSUB Cdf

560 MAT $\mathrm{Cd} 2=\mathrm{Cd}$

570 GOSUB Resfile ! storing cdf data in result file

$580 \mathrm{Nex}=2$

590 Cdf121:RETURN

1040

1050 ! doing cdf for joint event ( selection combining)

1060 Jnt12:GOSUB Getresf

1070 GOSUB Pdfbdry

1080 DISP " doing joint PDF of P1 and P2 ",Name\$

$1090 \mathrm{M} 1=\mathrm{MIN}\left(\mathrm{P} 1\left(^{*}\right)\right)$

1100 MAT SEARCH X1,LOC(>=M1);St1

$1110 \mathrm{M} 2=\operatorname{MIN}\left(\mathrm{P} 2\left(^{*}\right)\right)$

1120 MAT SEARCH X2,LOC(>=M2);St2

1121 ! The joint event will always be at least greater than the largest minimum of each P1 or P2.

$1130 \mathrm{St}=\mathrm{MAX}(\mathrm{St} 1, \mathrm{St} 2)$

1140 MAT Pdjt $=(0)$

1150 FOR I=1 TO Total STEP 2

1160 MAT SEARCH $X 1, \mathrm{LOC}(>=\mathrm{P} 1(\mathrm{I})) ; \mathrm{D} 1, \mathrm{St}$

1170 FOR J=D1 TO 41

1180 IF $P 2(\mathrm{I})<=\mathrm{X} 2(\mathrm{~J})$ THEN

$1190 \operatorname{Pdjt}(\mathrm{J})=\operatorname{Pdj}(\mathrm{J})+1$

1200 GOTO Jnt2 
1210 END IF

1220 NEXT J

$1230 \operatorname{Pdjt}(42)=\operatorname{Pdit}(42)+1$

1240 Jnt2:MAT SEARCH X1,LOC(>=P1(I+1)); D1,St

1250 FOR $\mathrm{J}=\mathrm{D} 1$ TO 41

1260 IF $\mathrm{P} 2(\mathrm{I}+1)<=\mathrm{X} 2(\mathrm{~J})$ THEN

$1270 \operatorname{Pdjt}(\mathrm{J})=\operatorname{Pdjt}(\mathrm{J})+1$

1280 GOTO Jnt3

1290 END IF

1300 NEXT $J$

$1310 \operatorname{Pdjt}(4 ?)=\operatorname{Pdjt}(42)+1$

1320 Jnt3:NEXT I

1330 DISP "doing CDF",Name\$

1340 MAT $\mathrm{Pd}=$ - djt

1350 GOSUB Cdf

1360 MAT Cdjt= Cd

$1370 \mathrm{Nex}=2$

1380 GOSUB Resfile

$1390 \mathrm{Jnt} 121:$ RETURN

2880

$2890 !$

2900 Cdf:! general routine to do CDF

2910 DISP "DOING CDF"

$2920 \mathrm{MAT} \mathrm{Pd}=\mathrm{Pd} /($ Total $)$

2930 MAT $\mathrm{Cd}=(0)$

$2940 \mathrm{Cd}(1)=\mathrm{Pd}(1)$

2950 FOR $\mathrm{l}=2$ TO 42

$2960 \mathrm{Cd}(\mathrm{I})=\mathrm{Cd}(\mathrm{I}-\mathrm{I})+\mathrm{Pd}(\mathrm{I}) * \mathrm{Inc}$

2970 NEXT 1 
2980 DISP "DONE"

2990 Cdf:RETURN

3000 !

3010 !

3020 Pdf:MAT Pdl $=(0)$

2030 MAT $\mathrm{Pd} 2=(0)$

s. 10 GOSUB Pdfbdry

3050 DISP "doing PDF for P1 and P2",Name\$

3060 IF St $1<>0$ AND St $2<0$ THEN GOTO Pdf 1

$3070 \mathrm{M} 1=\mathrm{MIN}(\mathrm{P} 1(*))$

3080 MAT SEARCH X1,LOC(>=M1);St1

$3090 \mathrm{M} 2=\mathrm{MIN}\left(\mathrm{P} 2\left(^{*}\right)\right)$

3100 MAT SEARCH X2,LOC(>=M2);St2

3110 Pdf1:FOR I=1 TO Total STEP 2

3120 MAT SEARCH X1,LOC(>=P1(I));D1

$3130 \operatorname{Pd} 1$ (D1) $=\operatorname{Pd} 1(\mathrm{D} 1)+1$

3140! MAT SEARCH X2,LOC(>=P2(I));D2

3150! Pd2(D2)=Pd2(D2)+1

3160 MAT SEARCH X1,LOC(>=P1(I+1));D1

$3170 \operatorname{Pd} 1(\mathrm{D} 1)=\operatorname{Pd} 1(\mathrm{D} 1)+1$

3180! MAT SEARCH X2,LOC(>=P2(I+1));D2

3190! Pd2(D2) $=$ Pd2(D2)+1

3200 NEXT I

3210 DISP "DONE"

3220 Pdfl:RETURN

3230 !-

3240 !

3870 Pdfbdry:Inc $=1$

3880 DISP "Calculating,boundary values for the PDF ave-30 to ave +10 " 


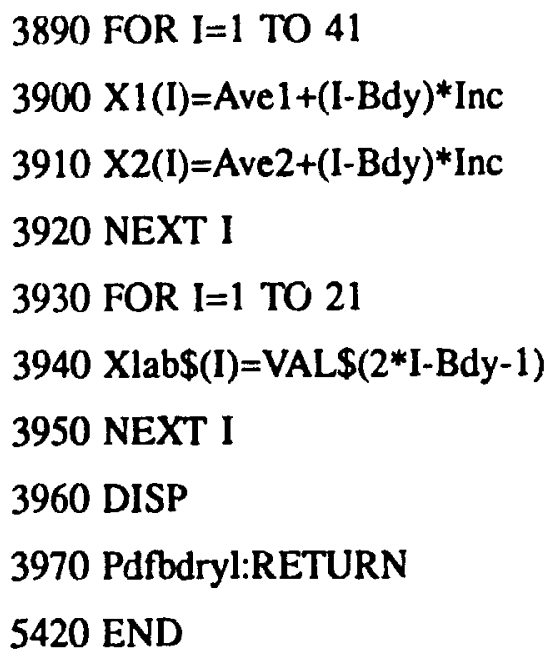

\section{A.5 LOSS}

This program does the calculation of the path losses. These losses were used to produce the scatter diagrams in section 4.4 .

100 ! LOSS: PROGRAM TO CALCULATE TRANSMISSION LOSS 200 ! FROM TX TO RX. 300 OPTION BASE 1

The three vectors Fatten, Satten, Patten have the data required to do the calculation. For all locations, the transmitter attenuator and the average received power in channel 1 and 2 is recorded in these veciors.

400 REAL Dist(25),Fatten(20,5,3),Satten(20,5,3),Patten(20,1,3)

The vectors Sptxpwr and Ftxpwr contain the power values corresponding to the attenuator vaiues for the two types of transmitters: frequency diversity and space/polarization diversity.

500 REAL Sptxpwr(-10:50), Ftxpwr(5,0:50,2),Spathloss(20,5,2),Ppathloss(20,1,2), 
Fpathloss $(20,5,2)$

600 Vol\$ $=": C S 80,151), 7 "$

The file Atten contains all the vectors needed.

700 ASSIGN @F1 TO "ATTEN:CS80,1500,7"

800 ENTER @F1;Fatten $\left({ }^{*}\right), \operatorname{Satten}\left({ }^{*}\right), P a t t e n\left({ }^{*}\right), D i s t\left({ }^{*}\right)$, Sptxpwr( $\left.{ }^{*}\right)$, Ftxpwr( $\left({ }^{*}\right)$

900 RESET @F1

1000 ! Path loss for space diversity, chan 1 and chan2

1100 FOR $\mathrm{K}=1$ TO 20

1200 FOR I $=1$ TO 5

Satten $(K, I, 1)$ is the attenuator value, Satten $(K, 1,2)$ is the average received power for channel 1 and Satten $(K, I, 3)$ is the average reieived power for channel

2.

1300 Spathloss $(K, 1,1)=\operatorname{Sptxpwr}(\operatorname{Satten}(\mathrm{K}, \mathrm{I}, 1))$-Satten $(\mathrm{K}, \mathrm{I}, 2)$

1400 Spathloss(K,I,2)= Sptxpwr(Satten(K,I,1))-Satten(K,I,3)

1500 NEXT I

1600 NEXT K

1700 ! Path loss for polarization diversity

1800 FOR K=1 TO 20

$1900 \mathrm{I}=1$

2000 Ppath!oss(K,I,1) = Sptxpwr(Patten(K,I,1))-Patten(K,I,2)

2100 Ppathloss(K,I,2)= Sptxpwr(Patten(K,I,1))-Patten(K,I,3)

2200 NEXT $K$

2300 ! FOR FREQ DIV

2400 FOR K=1 'TO 20

2500 FOR I $=1$ TO 5 
2500 Fpathloss $(K, I, 1)=F \operatorname{txpwr}(I, F a t t e n(K, I, 1), 1)-F a t t e n(K, I, 2)$

2700 Fpathloss $(K, I, 2)=\operatorname{Ftxpwr}(I, F a t t e n(K, I, 1), 2)-F a t t e n(K, I, 3)$

2800) NEXT I

2900 NEXT K

The file Pathloss contains the resulting vectors and the table of distance and location number.

3000 ASSIGN @P TO "PATHLOSS"\&Vol\$

3100 OUTPUT @P;Dist(*), Fpathloss(*), Spathioss(*), Ppathloss(*)

3200 END 


\section{Appendix B File Format}

\section{B.1 Data files}

The data files containing the collected data are configured in the following format: N\$, Sp, Total, Tim, D1(30000),D2(30000)

note: in addition of 1 byte per character, all alphanumeric (\$) variables have an extra 4 byte header plu's a pad byte at the end if they have an odd number of characters.

N\$: name of data file; 8 or 9 characters string; 14 bytes

Sp: spacing used in this file; from 1 to 5,0 used for polarization diversity; INTEGER, 2 bytes

Total: number of samples per channel ;REAL , 8 bytes, 30000 for all files

Tial: time of conversion to HP BASIC binary data file, REAL, 8 bytes

Dl(30000): vector containing raw data for channel 1, INTEGER, 2 bytes times $30000=60000$ bytes

D2(30000): vector containing raw data for channel 2, INTEGER, 2 bytes times $30000=60000$ bytes

\section{B.2 Result files}

The result files that were created when processing the data files had the following format:

N\$, Sp, Ave1, Ave2, Att, Cd1(42), Cd2(42), Cd3(42), Cdjt(42)

N\$: name of data file, same as for data file

Sp: spacing, same as for data file

Ave 1: average power of channel 1 , in $\mathrm{dBm}$; real REAL, 8 bytes

Ave2: average power of channel 2, in $\mathrm{dBm}$; REAL, 8 bylts 
Att: attenuator setting at the transmitter, in dB; REAL, 8 bytes

$\mathrm{Cd1}$ (42): cumulative distribution function for channel 1; REAL, $42 \times 8$ bytes

$\mathrm{Cd}$ (42): cumulative distribution function for channel 2; REAL, $42 \times 8$ bytes

Cd3(42): not used; REAL, $42 \times 8$ bytes

$\operatorname{Cdjt}(42)$ : cumulative distribution function for joint distribution; REAL, $42 \times$

8 bytes

\section{B.3 Result matrix files}

The result matrix file contains all the information from the respective result files collected in one large matrix. There are three files: FREQ_RES, SPAC_RES and POL_RES corresponding to the three diversity type. FREQ_RES and SPAC_RES have exactly the same format. Where information about 5 spacings is used for the previous two files, only one is used in FOL_RES. The files have the following format:

Typ\$, Validloc(20), Jnt $(20,5,42), \operatorname{Cuml}(20,5,42), \operatorname{Cum} 2(20,5,42)$, Locavejnt $(5,42$, 3), Locave1 $(5,42,3)$, Locave2(5, 42, 3)

Typ\$: type of diversity, 4 character string, 8 bytes;

Validloc(20): flag to indicate if given location is valid: 1 if valid, 0 if not; integer 40 bytes;

Jnt $(20,5,42)$ : matrix containing all joint cumulative distribution functions for 20 locations and 5 spacings; real, $4200 \times 8$ bytes;

Cum1 $(20,5,42)$ and $\operatorname{Cum} 2(20,5,42)$ : same as Jnt but for channel 1 and channel 2;

Locavejnt $(5,42,3)$ : point per point average of all cumulative distribution functions in Jnt. Locavejnt $(*, *, 1)$ is average value, Locavejnt $(*, *, 2)$ is the maximum value and Locavejnt $(*, *, 3)$ is the minimum value of all points Locave $1(5,42,3)$ and Locave2 $(5,42,3)$ : same as Locavejnt but for channel 1 and 2. 


\section{Appendix C DASH-16F}

\section{C.1 Features and options}

The main feature of this board is the Harris HI-774 12 bit successive approximation analog-to-digital converter (conversion time of $8 \mu \mathrm{s}$ ). Two input channels configurations are available: 16 single ended channels or $\mathbf{8}$ differential channels. Many input voltage ranges are available: $+1,+2,+5,+10$ volt unipolar or $\pm .5, \pm 1, \pm 2.5, \pm 5, \pm 10$ Volt bipolar. Conversions can be initiated in three ways: by software command, by the internal programmable timer, or by direct e ternal trigger. After the conversion is done, there are also three ways of transferring the data to the computer: using program transfer, using interrupts or by direct memory access (DMA). In addition to this analog-to-digital converter function, the board has two channels of multiplying 12 bit digital-to-analog output, a 4 bits TTL/DTL compatible output and 4 bits of digital input. This is a list of the 21 modes available on DASH-16F. Some are used for the analog-to-digital converter functions and some are used for the digital-to analog functions.

Mode 0:initialize the board

Mode 1: set multiplexer scan limits

Mode 2: read multiplexer scan limits

Mode 3: do $1 \mathrm{~A} / \mathrm{D}$ conversion and increment multiplexer

Mode 4: do N A/D conversions direct to array

Mnde 5: do N A/D conversions and transfer to memory on interrupt

Mode 6: do N A/D conversions and transfer to memory via DMA

Mode 7: disable DMA/interrupt operation

Mode 8: monitor status of operation

Mode 9: data transfer from memory to program

Mode 10: set configuration of counter 0 
Mode 11: load counter 0

Mode 12: read counter 0

Mode 13: write digital output line OPO-3

Mode 14: read digital output line IPO-3

Mode 15: output data to $1 \mathrm{D} / \mathrm{A}$

Mode 16: output dain to both D/A

Mode 17: set timer ratc

Mode 18: analog output/infut on interrupt

Mode 19: analog trigger function

Mode 20: do A/D on block of chinnel

The DASH-16F was only used as an analog-to-digital converter and we will now focus on the data acquisition modes

\section{C.2 Data acquisition modes}

There were many modes offered for the actual data açuisition. Different modes allowed much flexibility. We selected mode 20 because it fitter best our needs. In our case the ideal system should sample the channels simultaneously it the desired rate. A secondary requirement would be to keep the system as simple as possible. The following modes perform analog-to-digital conversion:

- mode 3: performs a single conversion, returns data and increments the multiplexer address. This is done under program control.

- mode 4: performs 1 conversion per trigger, up to total $\mathbf{N}$, on successive chaniels. The trigger is provided internally by the timer or externally. It transfers data directly to an array. The channels are scanned according to the limits set by mode 1.

- mode 5: performs 1 conversion per trigger, up to total N, on successive channels. The trigger is provided internally by the timer or externally. It transfers data to memory on interrupt. The channels are scanned according to limits set by mode 1 . 
- mode 6: same as mode 5 except that data transfer is done by DMA (direct memory access) to the specified memory location.

- mode 20: performs $\mathrm{C}$ conversions ( $=1$ scan) per trigger, up to total $\mathrm{N}$, on successive channels. $C$ is the number of channels specified by mode 1 . The trigger is provided by the timer or extemally. Data is transferred to memory by interrupt. The number of scan is $\mathrm{N} / \mathrm{C}$.

We will study in more detail the performance of each mode and explain why mode 20 was selected.

Mode 3 seems adequate but since each conversion is done under program control, it is too slow and will not be considered any further. Some limitations of the driver subroutine and the hardware must be highlighted before going any further. Without going into :00 much detail, the DMA page register ( 1 page $=64$ Kbytes of memory) cannot be incremented by the DMA controller. Any DMA data transfer is limited to a maximum of 64 Kbytes worth of data. Each value being 2 bytes, 32767 values is the maximum number of values that can be transferred in one attempt. In addition, the parameter $\mathrm{N}$ mentioned in the previous paragraph being a two bytes 2 's complement integer, any number greater than 32767 is treated as a negative number and returns an error in the FLAG parameter.

Mode 4, 5, and 6 are very similar in that they perform N ( $\leq 32767)$ conversions on $\mathrm{C}$ channels. The conversions are equally spaced in time by $1 /$ timer rate. The fastest conversion speed available on the DASH-16F is 100,000 samples/s (10 $\mu$ s between samples). As far as the signal envelope is concerned, nothing of significant importance happens faster than $10 \mu \mathrm{s}$, so this as good as simultaneous. At this rate, 1 second worth of data would occupy close to $200 \mathrm{~K} . \mathrm{b}$ of memory space, 30 seconds would use close to $6000 \mathrm{~Kb}$. This quickly iecomes unmanageable.

This brings us to the topic: of interval between sampling on each channel versus intarval between each pair of samples. Obviously the time between each samples should be short enu'igh so that simultaneous readings can be done on both channels. The time between each pan of sample might not need to be so short. Once this is taken into 
account, the solution offered by mode 20 looks promising. The DASH-16F manual states that with a $4.77 \mathrm{MHz}$ clock, a PC using mode 20 has a time interval between sampling on two successive channels of about $100 \mu \mathrm{s}$. The Vectra with a clock of 8 $\mathrm{MHz}$ should be faster.

To find out more precisely what was the time interval between the sampling of input channel 0 and channel 1, the following test was done. A sawtooth signal was fed to an oscilloscope. The slope of the rising part of the wave was calculated, in $\mathrm{mV} / \mathrm{ms}$. The sawtooth signal was then connected to the two channels' input. With a timer rate much faster than the signal frequency, a few hundred sample; were taken. The difference in readings between each channel for readings taken on the rising edge of the waveform was averaged. With the slope calculated earlier and the voltage range of the converter, the calculated time difference was $60 \mu \mathrm{s} \pm 10 \%$. This time was considered short enough to be satisfactory and mode 20 was selected for the data acquisition.

The analog-to-digital converter had many options available but once each one was understood, the choice between them was fairly obvious. Although with hardware and software limitations, the DASH-16F was a useful tool. The implementation of each option was demonstrated in the example programs provided with the board. The disa acquisition control program was written using as building blocks the various example programs provided and by adding the customized functions required by our specific application. 


\section{Appendix D Scatter Diagrams}

The following 8 scatter diagrams are the remainder of the diagrams not shown in chapter 4. For frequency diversity, spacing $5 \mathrm{MHz}, 10 \mathrm{MHz}, 15 \mathrm{MHz}$ and $20 \mathrm{MHz}$ are in figure D.1 to D.4. For space diversity, spacing $1 \lambda, 1.5 \lambda, 1.66 \lambda$ and $2 \lambda$ are shown in figure D.5 to D.8.

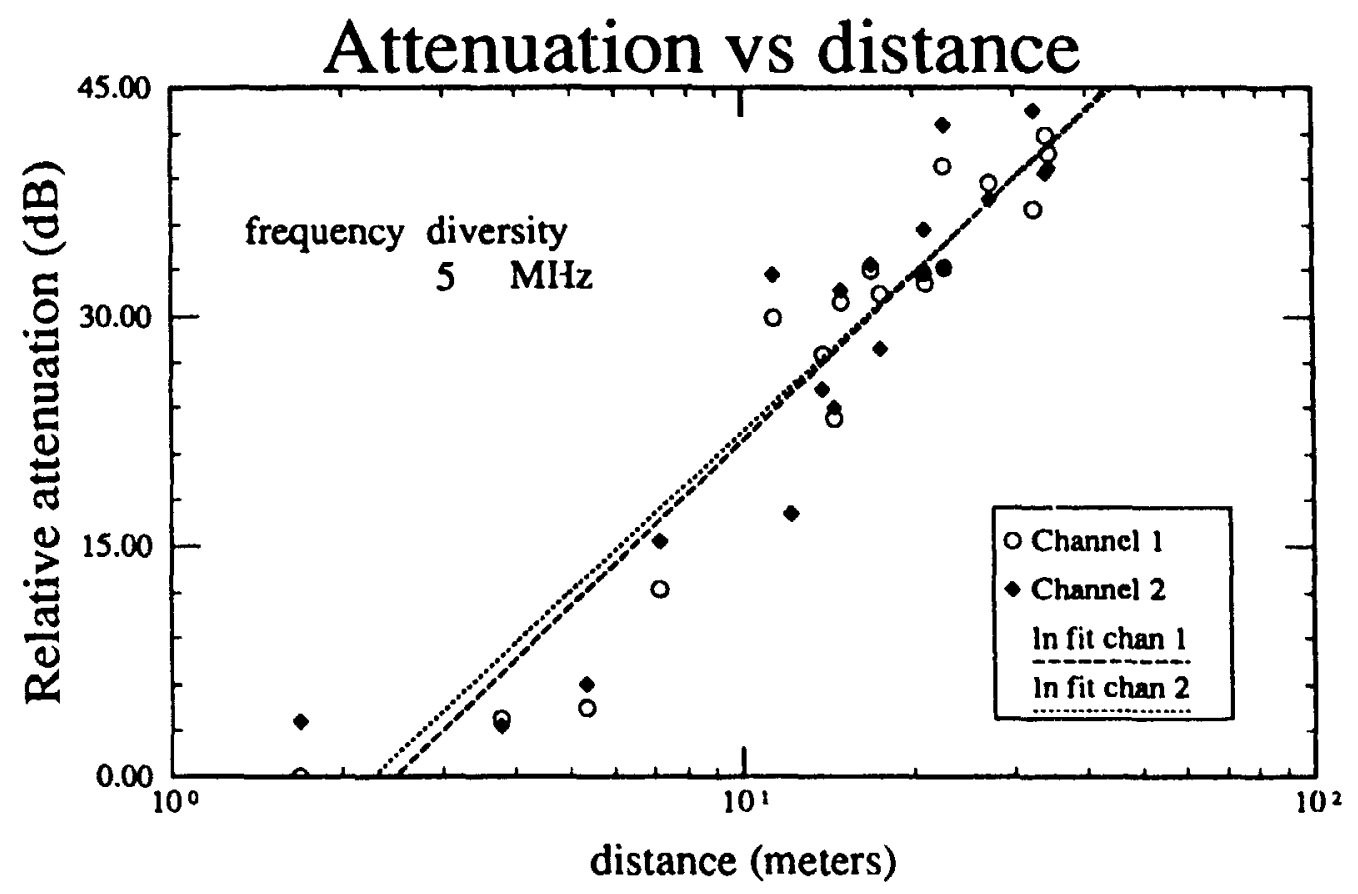

Figure- D.1: Scatter diagram for frequency diversity $S \mathrm{Mllz}$, all locations 


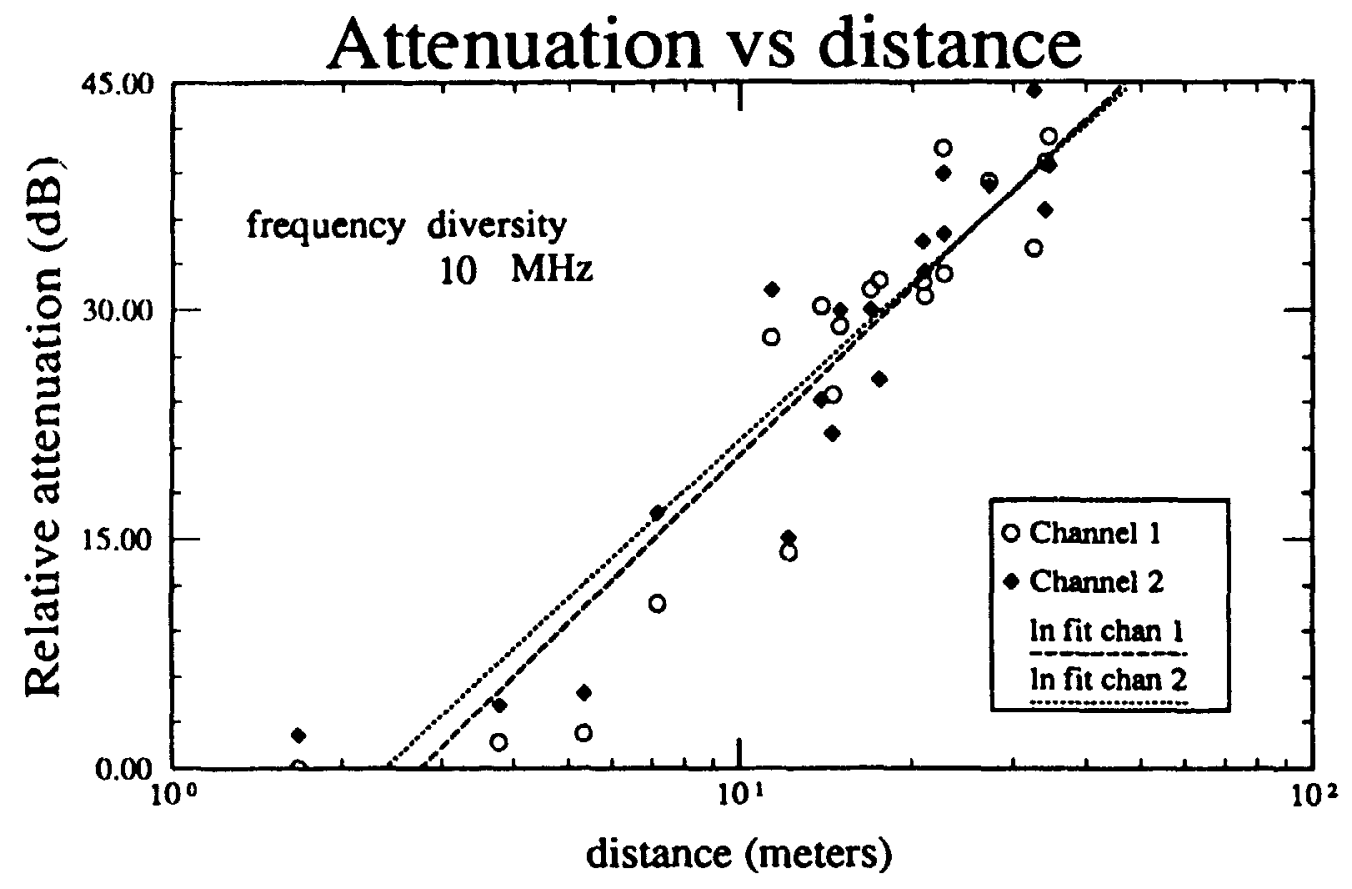

Figure- D.2: Scatter diagram for frequency diversity $10 \mathrm{MHz}$, all locations

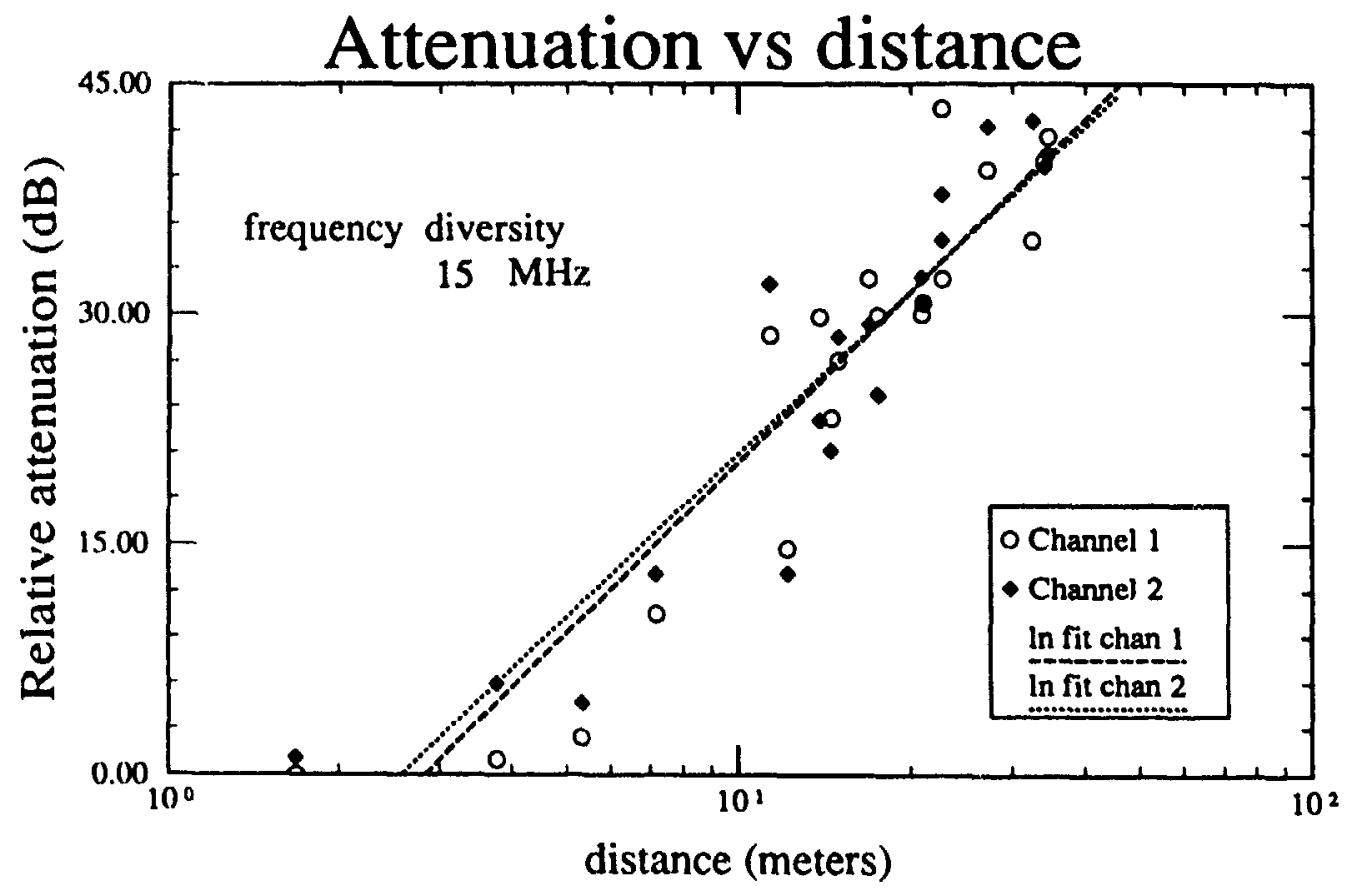

Figure- D.3: Scatter diagram for frequency diversity 15 MH2, all locations 


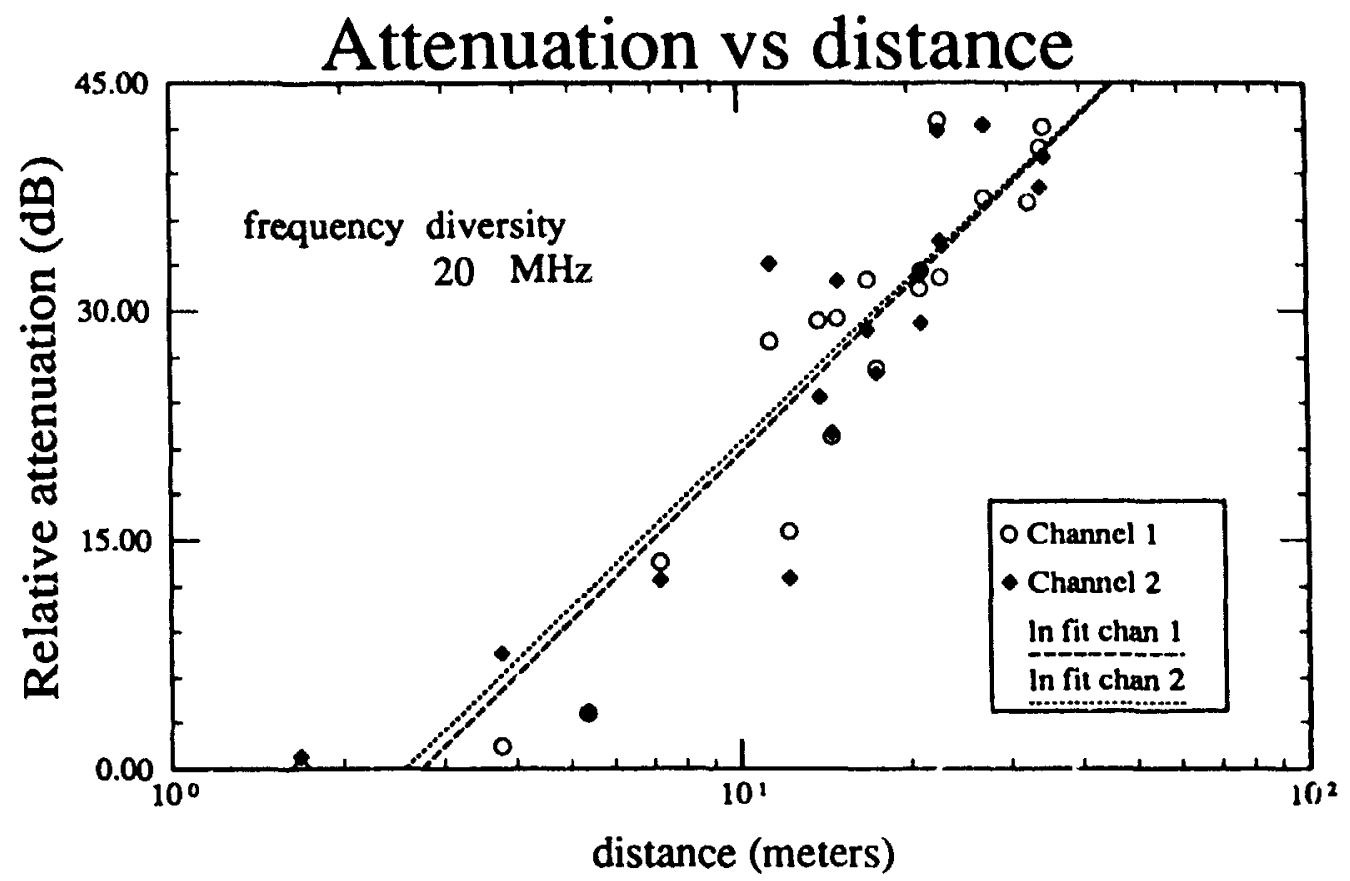

Figure- D.4: Scalter diagram for frequency diversity $20 \mathrm{MIL}$, all locations

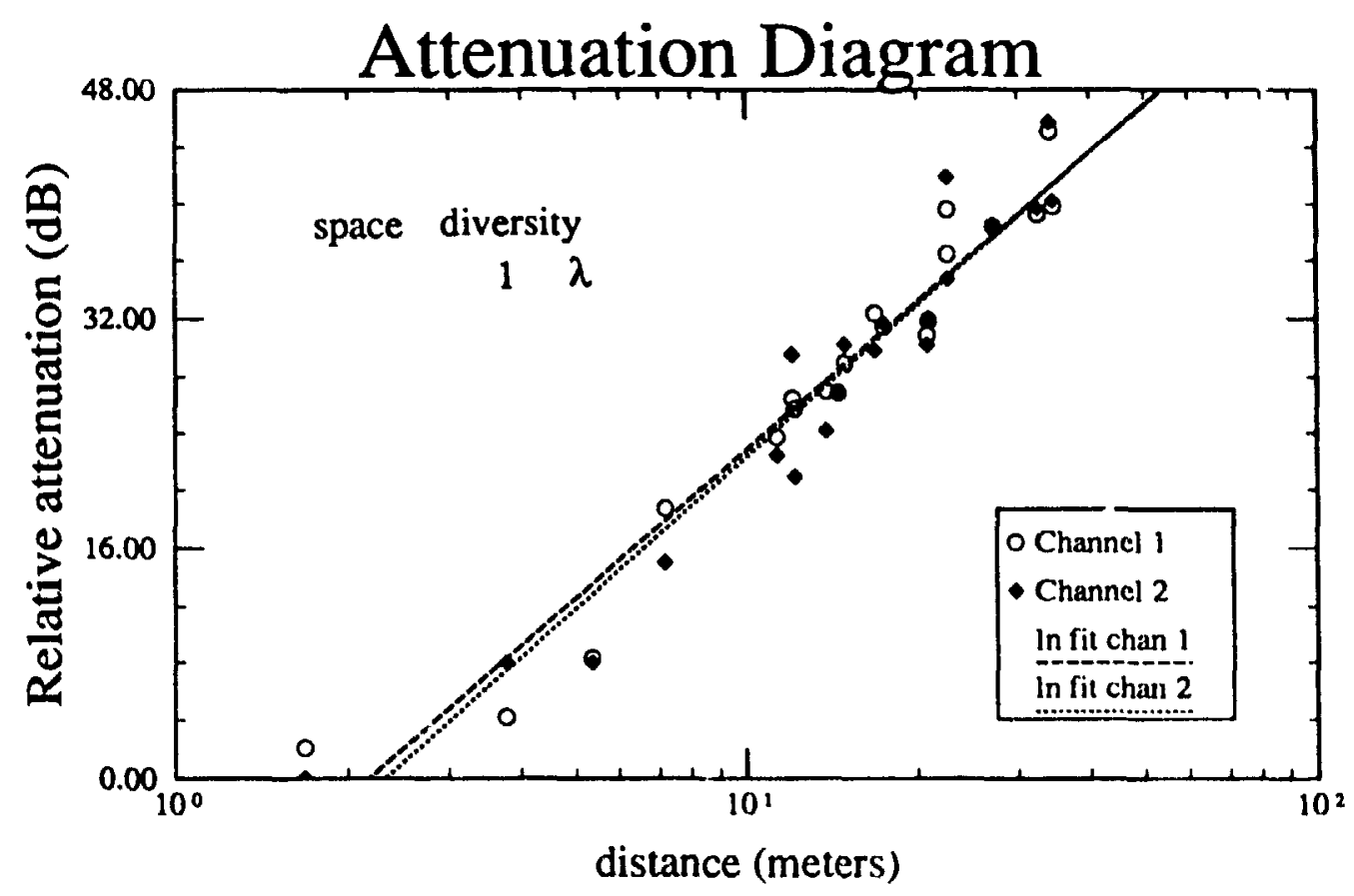

Figure- D.5: Scatter diagram for space diversity $1 \lambda$, all locations 


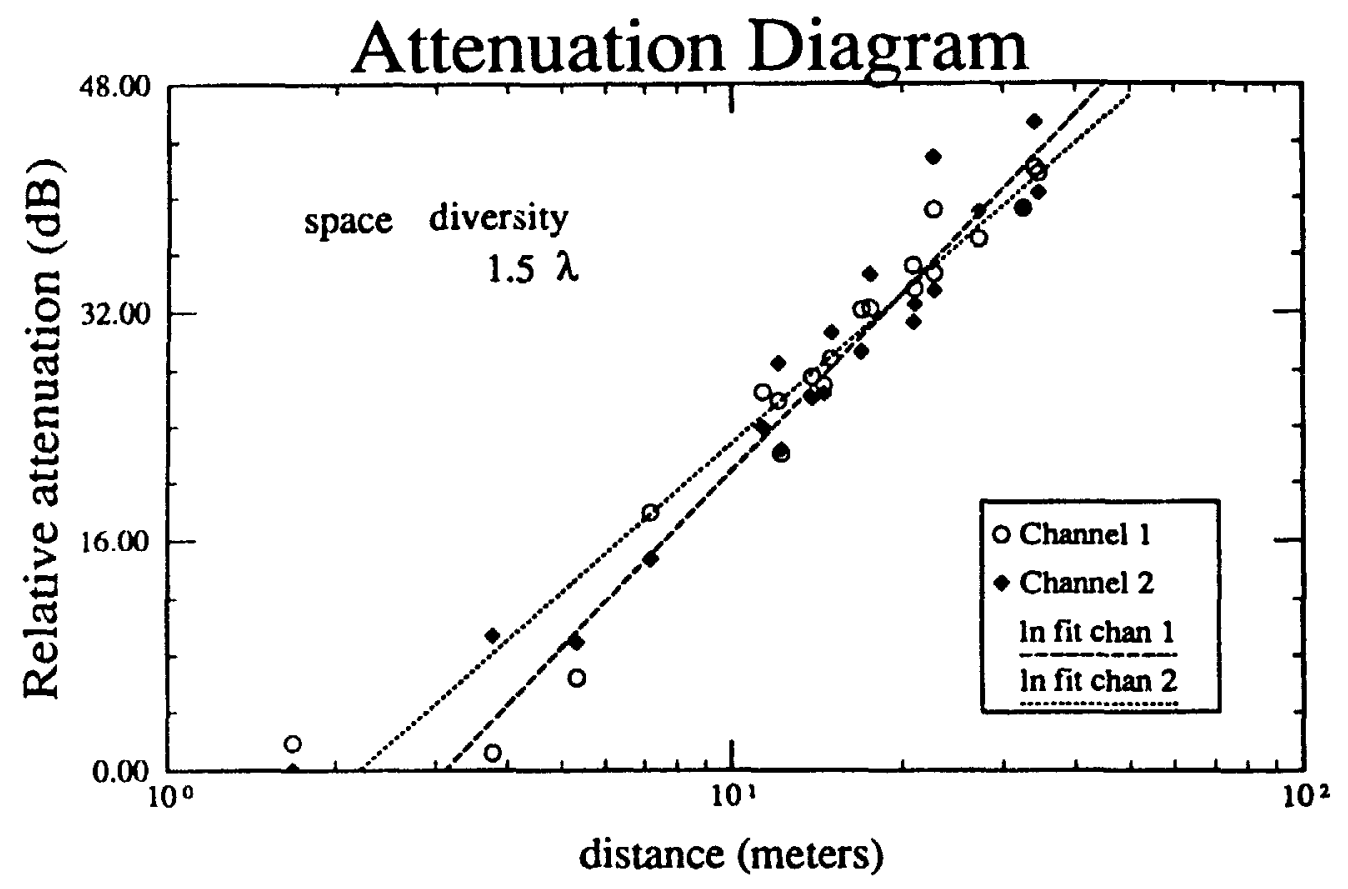

Figure- D.6: Scatter diagram for space diversity $1.5 \lambda$, all locations

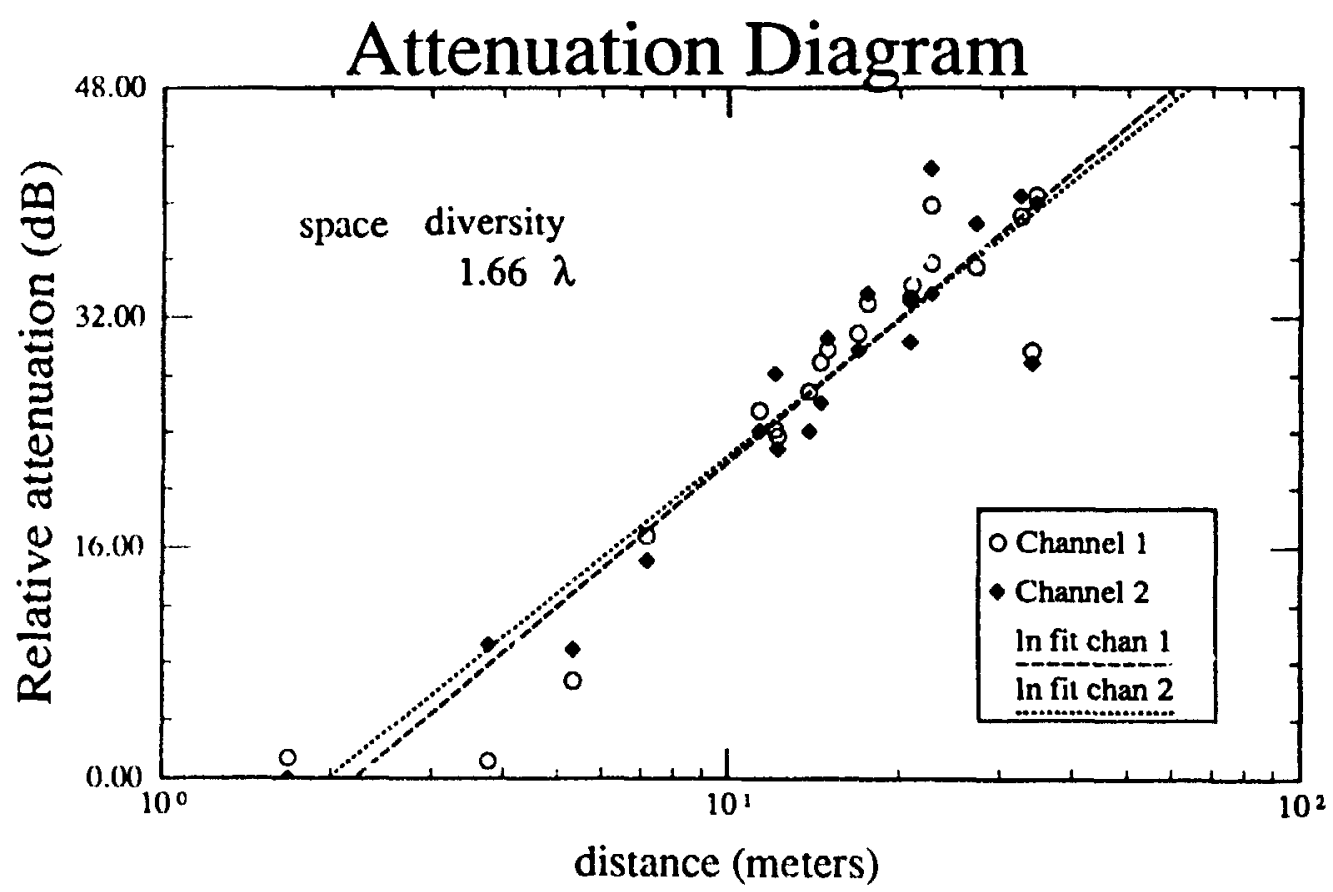

Figure- D.7: Scalter diagram for space diversity $1.66 \lambda$, all locations 


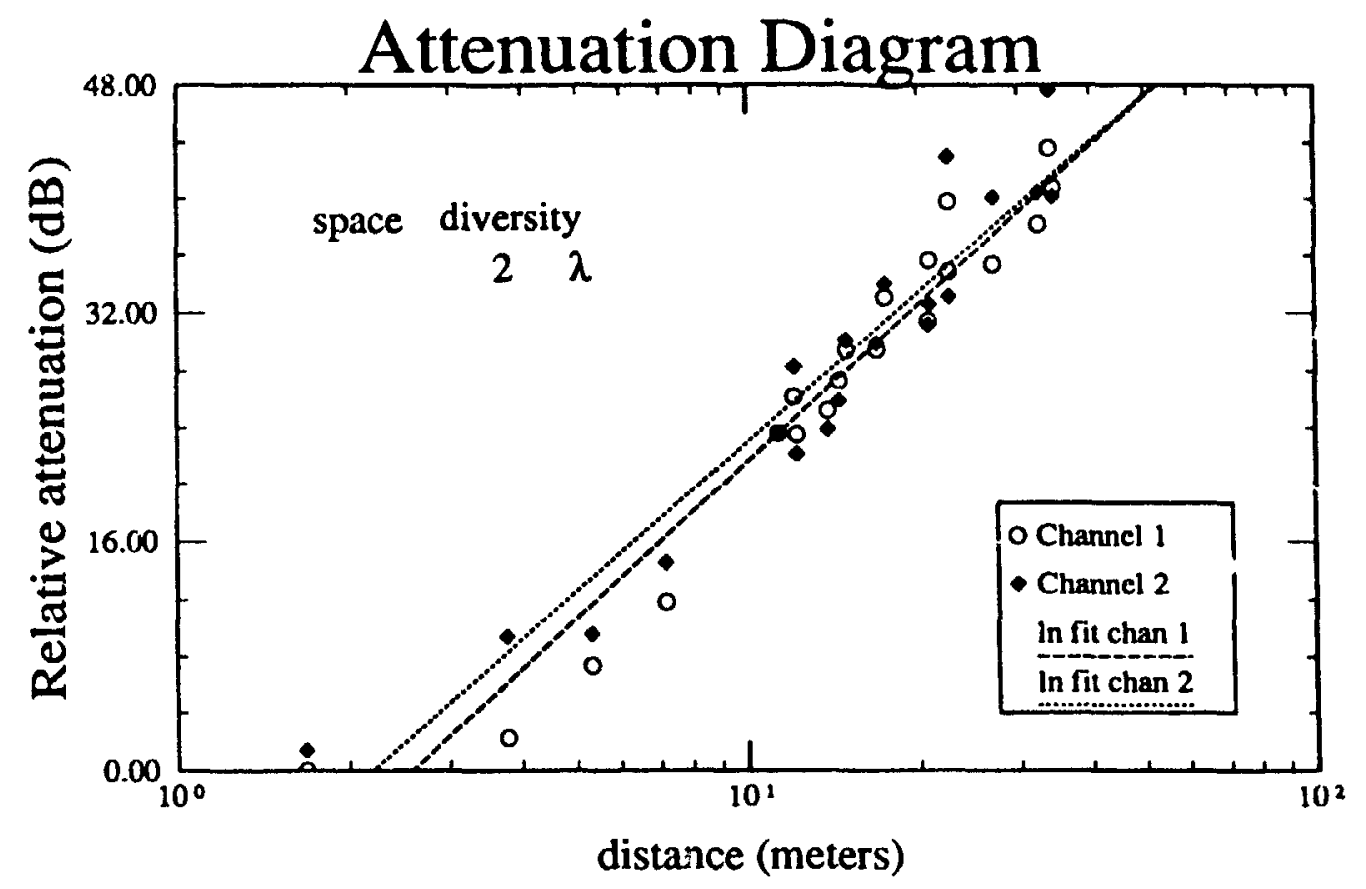

Figure- D.8: Scatter diagram for space diversity $2 \lambda$, all locations 


\section{Appendix E Measurement area and Conditions}

\section{E.1 Measurement locations}

Figure E.1 shows a floor plan of the area where the measurements were taken. The receiver (marked as $\mathrm{R}$ on the floor plan) was located in one of the laboratory and the transmitter was moved to all the locations marked by a number. Table E.1 shows the location number and the corresponding separation from the receiver.

\begin{tabular}{|c|c|c|c|}
\hline \multicolumn{4}{|c|}{ Locations and Distances } \\
\hline Locations & $\begin{array}{c}\text { distances } \\
\text { (meters) }\end{array}$ & Locations & $\begin{array}{c}\text { distances } \\
\text { (meters) }\end{array}$ \\
\hline 1 & 1.68 & 11 & 16.89 \\
\hline 2 & 3.78 & 12 & 22.68 \\
\hline 3 & 5.33 & 13 & 22.62 \\
\hline 4 & 7.19 & 5 & 20.85 \\
\hline 5 & 12.19 & 15 & 27.19 \\
\hline 6 & 12.07 & 16 & 34.14 \\
\hline 7 & 14.94 & 17 & 32.55 \\
\hline 8 & 20.97 & 18 & 11.34 \\
\hline 9 & 14.51 & 19 & 34.63 \\
\hline 10 & 13.84 & 20 & 17.50 \\
\hline
\end{tabular}

Table E.1 Locations and distances 


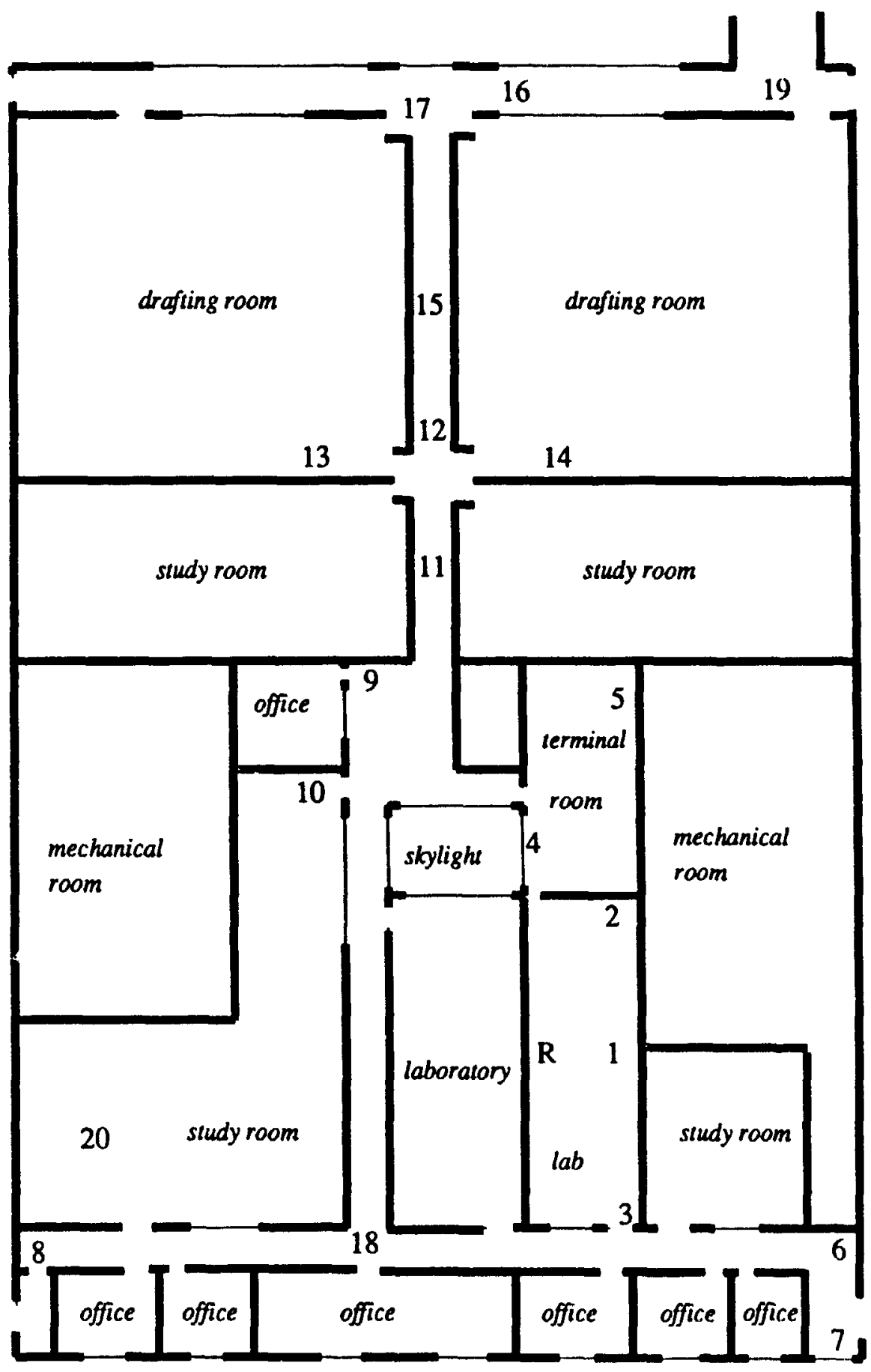

Figure- E.1: Floor plan of the measurement area and locations position 


\section{E.2 Measurement conditions}

The frequency diversity measurements were taken from 20 March to 30 March 89 from about $1200 \mathrm{hrs}$ to $1630 \mathrm{hrs}$ during the day to get the maximum of activity in the halls and the classrooms. Most of the time, the two classrooms were half to three quarter full. About half the offices were occupied at one moment or another. The study rooms were also occupied. In the lab where the receiver was located, the person taking the measurements was always present plus one or two more. The doors of that lab were regularly open and closed as people would go back and forth to the adjacent computer terminal room.

The space diversity measurements were taken from 5 to 14 April 89 during the same period of the day. The activity level was about the same. The polarization diversity measurements were taken from 21 to 24 April also during the same period of the day. The activity level at that time was lower then for the two previous sets of experiments. The study rooms were still used but the classrooms were close to empty most of the time. Less people were walking in the halls. The activity level in the receiver lab and the adjacent terminal room stayed the same.

\section{E.3 Hardware stability}

There is two aspects that this section will cover: the frequency stability of the synthesizers and the gain stability of the transmitter power amplifier. The synthesizers used had a stability of 1 in $10^{-7}$ : this meant that for a transmit frequency of $915 \mathrm{MHz}$, there could be a drift of $90 \mathrm{~Hz}$. Since the filter at the receiver had a bandwidth of 25 $\mathrm{kHz}$ and that we were using single tones only, the drift had no effect on the validity of the results.

After an initial warm up period (50 minutes), the gain of the transmitter power amplifier was stable within $.2 \mathrm{~dB}$. This warm up was done before every measurement. In addition, the diversity and the attenuation results were normalized so that variations in the absolute power level would not affect the results. The cumulative distributions 
were calculated relative to the average received power of each set of measurements. A variation in the absolute power would not affect the results. The attenuation results were calculated relative to the attenuation of the closest location $(1.72 \mathrm{~m})$. This also removed any effect of absolute power variations on the results. 

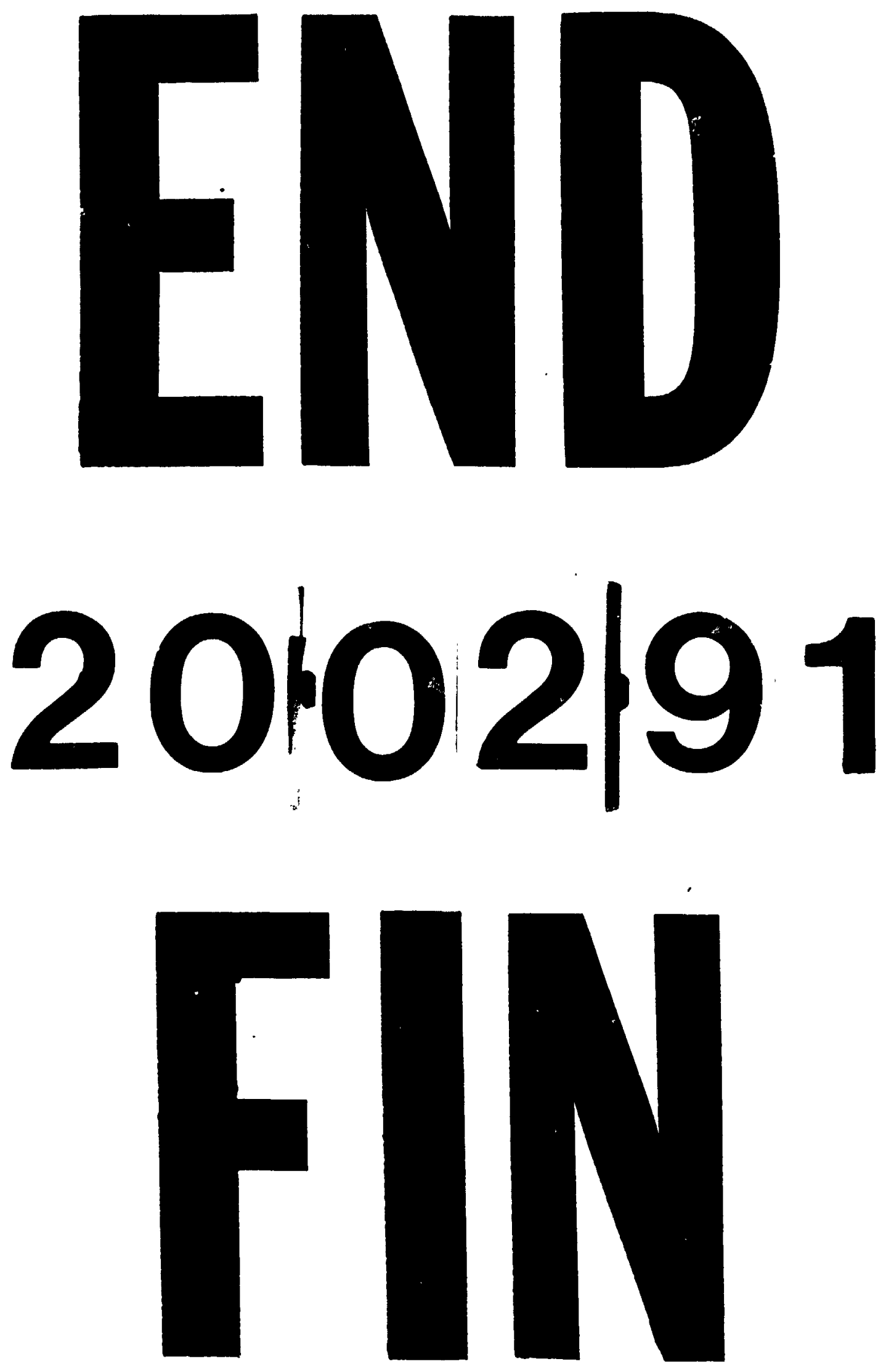